\title{
Search for Electroweak SUSY Signatures with Two Leptons and Missing Energy with the ATLAS Detector
}

\author{
by \\ Ryuichi (Rick) Ueno \\ A thesis submitted to the \\ Faculty of Graduate and Postdoctoral Affairs \\ in partial fulfillment of the requirements for the degree of \\ Doctor of Philosophy \\ in \\ Physics \\ Ottawa-Carleton Institute for Physics \\ Department of Physics \\ Carleton University \\ Ottawa, Ontario, Canada \\ December, 2014 \\ (C) copyright 2014 \\ Ryuichi (Rick) Ueno
}


ABstract

The ATLAS experiment at the Large Hadron Collider (LHC) has collected significant amounts of proton-proton $(p p)$ collision data from 2010 until 2012. These data have allowed the discovery of a new boson consistent with the one postulated by the Higgs' theory, and also enabled to look for hints of new physics beyond the Standard Model $(\mathrm{SM})$.

Supersymmetry (SUSY) is an extension of the SM that introduces a symmetry between fermions and bosons. Its signatures are expected to be detectable at the LHC, should it be the true extension to the SM, and the ATLAS experiment offers a dedicated programme to search for such signatures.

This thesis presents the analysis and results for the search for SUSY produced from electroweak interactions resulting in two final-state leptons and some missing energy. The full 2012 dataset is used for the analysis, corresponding to $20.3 \mathrm{fb}^{-1}$ of $p p$ collisions at the centre-of-mass energy of $\sqrt{s}=8 \mathrm{TeV}$. The overall methodology to identify these SUSY signatures is given, with emphasis on the optimization of the SUSY discovery signal region definitions and statistical interpretation of the results.

No significant excess of events above the SM prediction is observed in the signal 
regions. Therefore, the final result is interpreted by means of statistical tests designed to exclude regions of parameter space of specific SUSY models. For this thesis, the phenomenological Minimally Supersymmetric Standard Model (pMSSM) is tested, and exclusion limits are set on gaugino mass parameters $\left(M_{2}\right.$ and $\left.\mu\right)$. The exclusion regions are expanded since the previously published results based on the LHC $7 \mathrm{TeV}$ collision data. By combining these results with those from searches with trilepton final states, the exclusion regions are further expanded, giving the strongest limits on the gaugino mass parameters to date. 


\section{ACKNOWLEDGEMENTS}

First and foremost, I would like to thank my supervisor, Dr. Manuella Vincter, for her guidance and support throughout all of my post-graduate studies. Her depth of knowledge, enthusiasm and attention to detail make her, in my personal opinion, the best graduate supervisor one could ask for.

This thesis work could not have been completed without the researchers at CERN working towards the common goal: Dr. Pascal Pralavorio, who has guided me through a (relatively) short but significant part of my thesis work; Dr. Anyes Taffard and Dr. Serhan Mete, who showed me the importance and art of making eye-catching kinematic plots; Matt Relich for his very organized scripts that make the nightmareish software easy to use; and lastly but certainly not least, Dr. Géraldine Conti, who I considered as my supervisor outside of Carleton for her patience and guidance with the majority of the research project presented within this thesis.

A great experience is not attainable without great people surrounding it. The office mates at Carleton (Graham Cree, David DiValentino, Jim Lacey, Tom McCarthy, and Kate Whalen) have made my experience unforgettable, from working through nearly-impossible class assignments, to working through a fun brainteaser 
(innovative ways to cut pizza slices?) and of course providing hints and feedback whenever I needed them, no matter how stupid the question was.

Last but not least - my friends and family. My beautiful wife Kelly has always supported me, in highs and lows through this endeavour. There is no doubt that I am the person I am today because of you. My son Henry, for the great smile on his face that makes all the problems go away whenever I see it. My parents, for their interest in my research, and their effort to try to understand the subject (perhaps this thesis will be a better read than the Scientific American special issue). Finally, of course, my extended family and friends. Their enthusiam and interest in my research is equally influential to my academic career. One day, I will succeed to explain that I am not trying to make a black hole or a time machine. 



\section{TABle of Contents}

Abstract $\quad$ ii

Acknowledgments $\quad$ iv

Table of Contents $\quad$ V

List of Tables $\quad$ X

List of Figures $\quad$ xi

1 Introduction and Motivation $\quad 1$

1.1 The Standard Model . . . . . . . . . . . . . . . . . . . . 2

1.1.1 Brief Overview of the Standard Model . . . . . . . . . . . . 2

1.1.2 The Limitations of the Standard Model . . . . . . . . . . . . . 4

1.2 Supersymmetry . . . . . . . . . . . . . . . . 7

1.2.1 General Introduction . . . . . . . . . . . . . . . . 7

1.2.2 Particle Content in the MSSM . . . . . . . . . . . . . 8

1.2.3 Previous Searches for SUSY Signatures . . . . . . . . . . . . . 9

1.3 Objective of the Thesis . . . . . . . . . . . . . . . 10

1.4 Outline of the Thesis . . . . . . . . . . . . . . . 11

2 The ATLAS Detector at the LHC 13

2.1 The LHC . . . . . . . . . . . . . . . . . . . . 14

2.1.1 $p p$ Collision Terminology . . . . . . . . . . . . . 16 
2.2 The ATLAS Detector . . . . . . . . . . . . . . . . . . . 18

2.2.1 Description of ATLAS Subdetectors . . . . . . . . . . . 21

2.2.2 Collection of Data for Physics Analyses . . . . . . . . . . 25

3 Introduction to Electroweak SUSY Production $\quad 27$

3.1 MSSM Theory . . . . . . . . . . . . . . . . . 27

3.1.1 General SUSY Lagrangian . . . . . . . . . . . . . . 27

3.1 .2 Soft Supersymmetry Breaking . . . . . . . . . . . . . . 31

3.1 .3 MSSM At Electroweak Scale . . . . . . . . . . . . . 33

$3.1 .4 \quad$ R-Parity . . . . . . . . . . . . . . . . . . 35

3.2 Production Process . . . . . . . . . . . . . . . . . . . . 36

3.3 Final States . . . . . . . . . . . . . . . . . . . . . . . 37

3.4 SUSY Signal Models . . . . . . . . . . . . . . . . . . . . 40

$3.4 .1 \quad \mathrm{pMSSM} \ldots \ldots \ldots \ldots \ldots \ldots \ldots$

3.4 .2 Simplified Model . . . . . . . . . . . . . . . . . . 42

3.5 Standard Model Backgrounds . . . . . . . . . . . . . . . . 43

3.5 .1 Тор . . . . . . . . . . . . . . . . . . . 44

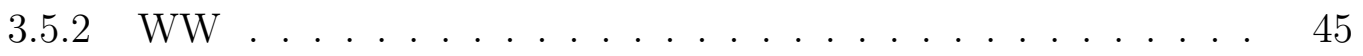

$3.5 .3 \mathrm{ZV}(\mathrm{ZZ} / \mathrm{ZW}) \ldots \ldots \ldots \ldots \ldots \ldots \ldots \ldots \ldots$

$3.5 .4 \quad \mathrm{ZX}(\mathrm{Z}+$ jets $) \ldots \ldots \ldots \ldots \ldots \ldots \ldots$

3.5.5 Higgs . . . . . . . . . . . . . . . . . . . . . . . 47

3.5 .6 Fake Leptons . . . . . . . . . . . . . . . . . 47

4 Methodology 48

4.1 Data and Monte Carlo Samples . . . . . . . . . . . . . . . . 49

4.1 .1 Data Collection . . . . . . . . . . . . . . . . . . 49

4.1 .2 Triggers . . . . . . . . . . . . . . . 50

4.1 .3 Monte Carlo Samples . . . . . . . . . . . . . . . . . . . 52

4.2 Object Definitions and Event Selection . . . . . . . . . . . 54

4.2 .1 Leptons . . . . . . . . . . . . . . . . . . . . 55

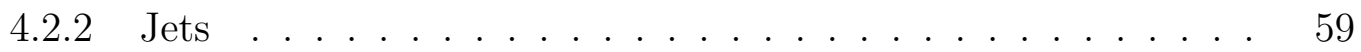

4.2 .3 Overlap Removal of Leptons and Jets . . . . . . . . . . . . . 61

4.2 .4 Missing Energy . . . . . . . . . . . . . . . . . . 62 
4.3 Analysis Strategy . . . . . . . . . . . . . . . . . . . 64

4.3 .1 Blinding of the Data . . . . . . . . . . . . . . . . 64

4.3.2 Signal Region Definition . . . . . . . . . . . . . . . 64

4.3.3 Estimation of the Backgrounds . . . . . . . . . . 65

4.3.4 Unblinding of the Data . . . . . . . . . . . . . . . 65

4.3.5 Statistical Interpretation . . . . . . . . . . . . 66

4.4 Systematic Uncertainties . . . . . . . . . . . . . . . . 67

4.4 .1 Jet Uncertainties . . . . . . . . . . . . . . . . . . . 67

4.4 .2 Lepton Uncertainties . . . . . . . . . . . . . . . . . . 68

4.4 .3 Missing Energy Uncertainties . . . . . . . . . . . . . 68

4.4 .4 b-tagging Uncertainties . . . . . . . . . . . . . . . . . . . 69

$4.4 .5 \quad$ Fake Lepton Uncertainties . . . . . . . . . . . . . . . . 69

4.4 .6 Modelling Uncertainty . . . . . . . . . . . . . . . . . . 69

4.4 .7 Luminosity Uncertainty . . . . . . . . . . . . . . 70

5 Signal Region Optimization $\quad 71$

5.1 Optimization of SR- $W W \ldots \ldots \ldots \ldots \ldots \ldots \ldots$

5.1 .1 Kinematic Variable Choices . . . . . . . . . . . . . 73

$5.1 .2 \mathrm{Zn}$ as a Figure of Merit . . . . . . . . . . . 76

5.1 .3 Cut Value Scan . . . . . . . . . . . . . . . . . . 77

5.2 Impact of Systematic Uncertainties on the Expected Sensitivity . . . 83

5.3 Summary of the Signal Regions _ . . . . . . . . . . . . . 86

6 Results and Interpretation $\quad 87$

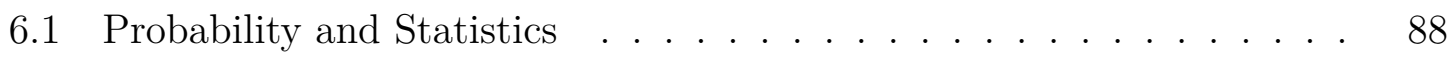

6.1 .1 Hypothesis Testing . . . . . . . . . . . . . . . 88

6.1.2 Profile Likelihood Ratio . . . . . . . . . . . . . . . . 90

6.1.3 Confidence Levels and the $C L_{s}$ Method . . . . . . . . . . . 91

6.2 Kinematic Distributions in Signal Regions . . . . . . . . . . . . 93

6.2.1 Kinematic Distributions in the Preselection Region . . . . . . 93

6.2 .2 Kinematic Distributions in SR- $m_{\mathrm{T} 2} \ldots \ldots \ldots$. . . . . . 97

6.2.3 Kinematic Distributions in SR- $W W \ldots \ldots \ldots$

6.2.4 Kinematic Distributions in SR-Zjets . . . . . . . . . . 101 
6.2.5 Event Visualization of Candidate Events . . . . . . . . . . . . 102

6.3 Background-Only Fit . . . . . . . . . . . . . . . . . . 106

6.3.1 Fit Results in the Control Regions . . . . . . . . . . . . . . . 106

6.3.2 Fit Results in the Signal Regions . . . . . . . . . . . . . . . . 109

6.3.3 Evaluation of the Systematic Uncertainties . . . . . . . . . . . 114

6.4 Model-Independent Fit (Discovery Fit) . . . . . . . . . . . . . . . . . 115

6.5 Model-Dependent Fit (Exclusion Fit) . . . . . . . . . . . . . . 116

6.5.1 pMSSM Direct Gaugino with Intermediate Sleptons . . . . . . 117

6.5.2 pMSSM Direct Gaugino without Intermediate Sleptons . . . 120

6.6 Combination with Trilepton Analysis . . . . . . . . . . . . . . 122

$\begin{array}{lll}7 & \text { Summary and Conclusions } & 126\end{array}$

Appendix A Personal Contributions to the ATLAS Collaboration 128

Appendix B List of Presentations Given 131

Appendix C List of Acronyms 133

$\begin{array}{ll}\text { References } & 135\end{array}$ 


\section{List OF TABLES}

1.1 List of fermions as defined by the Standard Model . . . . . . . . . . . 2

1.2 List of bosons as defined by the Standard Model . . . . . . . . . . . . 3

1.3 List of the MSSM Supermultiplets . . . . . . . . . . . . . . . . . 8

2.1 Relevant $p p$ collision information for ATLAS collision data . . . . . 26

4.1 Triggers used for the analysis . . . . . . . . . . . . . . . . 52

4.2 List of SM background MC samples . . . . . . . . . . . . . . . . . 54

4.3 List of SUSY signal MC samples . . . . . . . . . . . . . . . . 55

4.4 Definitions of lepton objects. . . . . . . . . . . . . 56

4.5 Definitions of jet objects . . . . . . . . . . . . . . . . 59

5.1 Summary of SR definitions . . . . . . . . . . . . . . . . 86

6.1 Background fit results in the CR for SR-m $m_{\mathrm{T} 2} \ldots \ldots$. . . . . . . . 107

6.2 Background normalization factors for SR- $m_{\mathrm{T} 2} \ldots \ldots$. . . . . . . . 107

6.3 Fitted background yield and the observed count in SR- $m_{\mathrm{T} 2}$. . . . . . 109

6.4 Fitted background yield and the observed count in SR- $W W$. . . . . 111

6.5 Fitted background yield and the observed counts in SR-Zjets . . . . . 113

6.6 Summary of systematic uncertainties . . . . . . . . . . . . . . . . . . 114

6.7 Result of model-independent fit . . . . . . . . . . . . . . . 116 


\section{List OF Figures}

1.1 Higgs' mass renormalization . . . . . . . . . . . . . . . 5

1.2 Rotation curves of galaxies . . . . . . . . . . . . . . . . 7

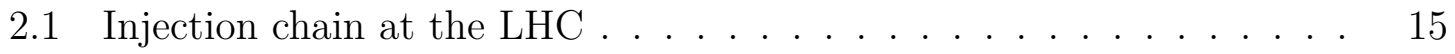

2.2 Schematic of an example $p p$ collision process . . . . . . . . . . . 16

2.3 An example visualization of a pile-up event. . . . . . . . . . . . . 18

2.4 Schematic of particle paths in a typical particle detector . . . . . . 19

2.5 The overall layout of the ATLAS detector . . . . . . . . . . . . . 19

2.6 The overall layout of the ATLAS ID system . . . . . . . . . . . . 21

2.7 The overall layout of the ATLAS calorimeter system . . . . . . . . . 23

2.8 The overall layout of the ATLAS muon system . . . . . . . . . . 23

2.9 Integrated luminosity and number of pile-ups in 2011 and 2012 . . . 25

3.1 Soft SUSY-breaking terms . . . . . . . . . . . . . . . . . 32

3.2 Example production processes of sparticles at the LHC . . . . . . . 37

3.3 Production cross sections of sparticles . . . . . . . . . . . . . . . 38

3.4 Decay chains of charginos and neutralinos . . . . . . . . . . . . 39

3.5 Dilepton signal topology for EW SUSY production . . . . . . . . . . 40

3.6 Examples of SM top production. . . . . . . . . . . . . . . 44

3.7 Examples of SM $W W$ production. . . . . . . . . . . . . . 45

3.8 Examples of SM $Z V$ production. . . . . . . . . . . . . . . 46

3.9 Examples of SM $Z+$ jets production . . . . . . . . . . . 46 
3.10 Examples of SM Higgs production . . . . . . . . . . . . . . . . 47

4.1 Summary of trigger regions . . . . . . . . . . . . . . . . 51

4.2 Illustration of impact parameter . . . . . . . . . . . . . . . . 57

4.3 Distribution of JVF. . . . . . . . . . . . . . . . . . . . . . 60

4.4 Illustration of missing transverse energy f . . . . . . . . . . . 63

4.5 Illustration of control regions . . . . . . . . . . . . . . . 66

5.1 Signal topology for EW SUSY production and Higgs production . . . 74

$5.2 m_{\mathrm{T} 2}$ distribution for top, $W W$, and two signals $\ldots \ldots . . . . . .75$

5.3 The optimal cut value on $p_{\mathrm{T}, \ell \ell}$ and $E_{\mathrm{T}}^{\mathrm{miss}, \mathrm{rel}} \ldots \ldots \ldots$. . . . . . . 79

5.4 The optimal cut value on $m_{\mathrm{T} 2}$. . . . . . . . . . . . . . . . . . . . . . 79

5.5 Scan of optimal $m_{\ell \ell}$ cut values . . . . . . . . . . . . . . . . . 81

5.6 Scan of optimal $\Delta \phi_{\ell, \ell}$ cut values . . . . . . . . . . . . . . . . . 81

5.7 Scan of optimal $p_{\mathrm{T}}^{\mathrm{MET}, \ell \ell}$ cut values . . . . . . . . . . . . . . . 82

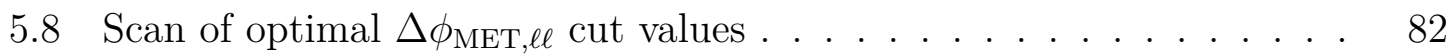

5.9 Impact on $Z_{n}$ significance due to systematics . . . . . . . . . . . . . . 84

5.10 Approximation of $95 \% \mathrm{CL}$ on the cross section for $\mathrm{m}\left(\tilde{\chi}_{1}^{0}\right)=0 \mathrm{GeV}$. . $\quad 85$

6.1 An example illustration of a critical region . . . . . . . . . . . . 89

6.2 Illustration of probability densities . . . . . . . . . . . . . . 92

6.3 Kinematic distributions in the preselection region for the SF channel 95

6.4 Kinematic distributions in the preselection region for the DF channel 96

6.5 Kinematic distributions of $m_{\mathrm{T} 2}$ in the SR- $m_{\mathrm{T} 2}$ a region . . . . . . . . . 97

6.6 Kinematic distributions of $m_{\mathrm{T} 2}$ in the SR- $m_{\mathrm{T} 2} \mathrm{~b}$ region . . . . . . . . 98

6.7 Kinematic distributions of $m_{\mathrm{T} 2}$ in the SR- $m_{\mathrm{T} 2} \mathrm{c}$ region . . . . . . . . . 98

6.8 Kinematic distributions of $E_{\mathrm{T}}^{\mathrm{miss}, \mathrm{rel}}$ in the $\mathrm{SR}-W W$ a region . . . . . . 99

6.9 Kinematic distributions of $E_{\mathrm{T}}^{\mathrm{miss}, \mathrm{rel}}$ in the $\mathrm{SR}-W W \mathrm{~b}$ region . . . . . 100

6.10 Kinematic distributions of $E_{\mathrm{T}}^{\mathrm{miss}, \mathrm{rel}}$ in the SR- $W W \mathrm{c}$ region . . . . . . 100

6.11 Kinematic distribution of $E_{\mathrm{T}}^{\mathrm{miss}, \mathrm{rel}}$ in the SR-Zjets region . . . . . . 101

6.12 Visualization of an event in the ee channel . . . . . . . . . . . . . . 103

6.13 Visualization of an event in the $\mu \mu$ channel . . . . . . . . . . . . . 104

6.14 Visualization of an event in the $e \mu$ channel . . . . . . . . . . . . . . . 105

6.15 Stacked histograms of background-only fit in the CR for SR- $m_{\mathrm{T} 2}$. . . 108 
6.16 Pull plots for SR- $m_{\mathrm{T} 2} \ldots \ldots \ldots \ldots \ldots$

6.17 Pull plots for SR- $W W \ldots \ldots \ldots \ldots$

6.18 Pull plot for SR-Zjets . . . . . . . . . . . . . . . . 113

6.19 Exclusion plots for pMSSM DG $M_{1}=100 \mathrm{GeV} \ldots \ldots \ldots \ldots$

6.20 Exclusion plots for pMSSM DG $M_{1}=140 \mathrm{GeV} \ldots \ldots \ldots \ldots$

6.21 Exclusion plots for pMSSM DG $M_{1}=250 \mathrm{GeV} \ldots \ldots \ldots \ldots$

6.22 Exclusion plots for pMSSM DG without intermediate sleptons . . . . 121

6.23 Trilepton topologies for EW SUSY production . . . . . . . . . . 122

6.24 Combined exclusion plot for pMSSM DG $M_{1}=250 \mathrm{GeV} \ldots \ldots \ldots$

6.25 Combined exclusion plot for pMSSM DG without intermediate sleptons 125 



\section{INTRODUCTION AND MOTIVATION}

Particle physics is the study of the most basic particles, the building blocks of our universe, and how they interact with each other via fundamental forces. Studies of these particles therefore deepen our understanding of the inner workings of nature.

The recent discovery at the Large Hadron Collider (LHC) of a particle consistent with that predicted by the Brout-Englert-Higgs mechanism (hereafter referred to as

the "Higgs") [1, 2] has received much attention from the general public, and as a consequence, an interest in particle physics research has spread. A broader range of the public has an understanding of general particle physics theory, which helps to motivate researchers to continue their quest to probe the unexplored domains of particle physics.

In this chapter, a general overview of the current working theory, called the Standard Model (SM) of particle physics, is given. A general overview of one of the more attractive extensions of the SM, called Supersymmetry, is subsequently provided. An outline of this thesis is presented at the end of the chapter. 


\begin{tabular}{|c|c|c|}
\hline \multicolumn{3}{|c|}{ Quarks $(\operatorname{spin}=1 / 2)$} \\
\hline Flavour & Mass $[\mathrm{GeV}]$ & Charge $[e]$ \\
\hline $\mathbf{u} \quad$ up & 0.003 & $2 / 3$ \\
\hline d down & 0.005 & $-1 / 3$ \\
\hline c charm & 1.27 & $2 / 3$ \\
\hline s strange & 0.101 & $-1 / 3$ \\
\hline $\mathbf{t}$ top & 175 & $2 / 3$ \\
\hline b bottom & 4.3 & $-1 / 3$ \\
\hline
\end{tabular}

\begin{tabular}{|c|c|c|}
\hline \multicolumn{3}{|c|}{ Leptons $(\operatorname{spin}=1 / 2)$} \\
\hline Flavour & Mass $[\mathrm{GeV}]$ & Charge $[e]$ \\
\hline$\nu_{e}$ electron & $<1 \times 10^{-8}$ & 0 \\
\hline $\boldsymbol{e} \quad$ electron & 0.000511 & -1 \\
\hline $\begin{array}{ll}\boldsymbol{\nu}_{\boldsymbol{\mu}} & \overline{\text { mūon }} \\
\text { neutrino }\end{array}$ & $<0.0002$ & 0 \\
\hline $\boldsymbol{\mu} \quad$ muon & 0.106 & -1 \\
\hline $\begin{array}{ll}\boldsymbol{\nu}_{\boldsymbol{\tau}} & \text { tau } \\
\text { neutrino }\end{array}$ & $<0.002$ & 0 \\
\hline $\boldsymbol{\tau} \quad \mathrm{tau}$ & 1.777 & -1 \\
\hline
\end{tabular}

Table 1.1: List of fermions as defined by the Standard Model. Fermions are the constituents of all matter, and have spin=1/2. They are further categorized as either quarks (left) or leptons (right). From Reference [3].

\subsection{The Standard Model}

\subsubsection{Brief Overview of the Standard Model}

The interplay of theoretical developments and experimental results since the 1930's has resulted in a theory that attempts to organize and categorize fundamental particles into groups based on their physical properties. This theory, known as the Standard Model (SM), encompasses twelve elementary particles (and their chargeconjugate states) that are the constituents of all matter, and four additional particles that act as the force mediators. The SM encompasses three of the four fundamental forces of nature: electromagnetic force (responsible for electricity and magnetism), weak force (responsible for weak nuclear decay), and strong force (responsible for binding quarks in nuclei). The categorization of these particles is shown in Tables 1.1 and 1.2. 


\begin{tabular}{|c|c|c|c|c|c|c|c|}
\hline \multicolumn{4}{|c|}{ Gauge Bosons $(\operatorname{spin}=1)$} & \multicolumn{4}{|c|}{ Scalar Bosons $(\operatorname{spin}=0)$} \\
\hline Name & & Mass $[\mathrm{GeV}]$ & Charge $[e]$ & $\mathrm{Na}$ & & Mass $[\mathrm{GeV}]$ & Charge $[e]$ \\
\hline$\gamma$ & photon & 0 & 0 & & Higgs boson & 125.3 & 0 \\
\hline $\mathbf{W}^{ \pm}$ & $\mathrm{W}^{ \pm}$boson & 80.4 & \pm 1 & & & & \\
\hline $\mathbf{Z}$ & $\mathrm{Z}$ boson & 91.2 & 0 & & & & \\
\hline $\mathrm{g}$ & gluon & 0 & 0 & & & & \\
\hline
\end{tabular}

Table 1.2: List of bosons as defined by the Standard Model. The gauge bosons (left) have spin=1, and they are the force-carriers, while the scalar bosons (right) have spin=0. From Reference [3].

The matter particles, known as fermions since they have an intrinsic spin of $1 / 2^{1}$, are further categorized into two groups: quarks and leptons. Quarks carry a charge called colour, which allow them to participate in inter-nuclear interactions via the strong interaction, while leptons are colourless. Quarks carry electric charge, in units of electron charge $e$, of either $2 / 3$ (up-type) or $-1 / 3$ (down-type). Leptons carry electric charge, in units of $e$, of either -1 or no charge, where the latter are called neutrinos. Both quarks and leptons have charge conjugate states with inverse electric charge, or inverse of other quantum numbers such as weak isospin, lepton numbers or colour charge, and they are denoted by a prefix "anti-".

The force mediator particles of the electromagnetic, weak and strong forces are called gauge bosons, and have intrinsic spin of 1 . They describe the nature of the interactions between the fermions. The electromagnetic interaction is described by a theory called Quantum Electrodynamics (QED) $[4,5,6]$, and is mediated by an exchange of photons. The strong interaction is described by Quantum Chromodynamics (QCD) $[7,8,9]$, and is mediated by the exchange of gluons. The strong interaction

\footnotetext{
${ }^{1}$ This thesis uses natural units where the Planck constant $\hbar$ and the speed of light $c$ are by definition equal to unity
} 
affects only the coloured particles, i.e. quarks. Lastly, the weak interaction follows the electroweak (EW) theory $[10,11,12]$, and is mediated by the $W^{ \pm}$and $Z$ bosons.

The EW theory alone predicts that the weak force mediators are massless, but this contradicts the existing measurements of the masses of the $W^{ \pm}$and $Z$ bosons [3]. The Higgs' mechanism [13] was introduced in order to address this mass issue. This mechanism gives masses to the $W^{ \pm}$and the $Z$ bosons while keeping the photons and the gluons massless. The same mechanism also gives fermions their appropriate masses. A consequence of the Higgs' mechanism is the existence of a massive particle called the Higgs' boson, $H$ (See Table 1.2). In 2012, it became the most recent SM particle to be experimentally observed when the ATLAS and the CMS experiments at the LHC (further explained in Chapter 2) made this significant observation [1, 2], consistent with the predictions of the theory.

\subsubsection{The Limitations of the Standard Model}

The SM has so far been very successful in describing the observations of nearly all particle physics experiments. However, the theory does not address some elements at all (for example the notion of gravity), and hence leaves some inconsistencies in the fine theoretical details as well as some of the cosmological observations. An example of each is described below.

\section{Hierarchy Problem}

The SM does not encompass gravitational interactions, so it is said to be an effective theory at the EW scale, defined by the $W$ boson mass $m_{W}=80 \mathrm{GeV}$. Gravity is described at the Planck scale $m_{P}=\left(8 \pi G_{\text {Newton }}\right)^{-1 / 2}=10^{18} \mathrm{GeV}$, which is 16 orders of magnitude larger [14, 15]. 


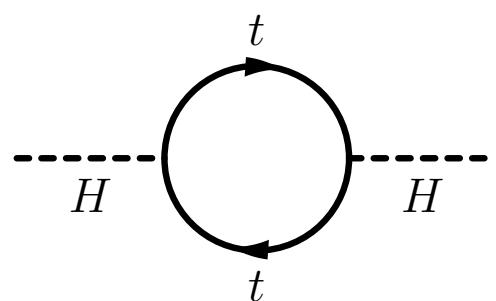

(a)

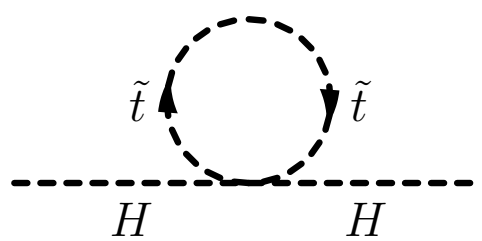

(b)

Figure 1.1: Higgs' quadratic mass renormalization of a top loop (a) and its superpartner, the stop (b). The renormalization of the stop loop can solve the hierarchy problem described in the text.

It is now experimentally known that the mass of the Higgs' boson is $m_{H} \simeq$ $125 \mathrm{GeV}$ [16]. However, the Higgs' mass is corrected by loop diagrams for each of the particles it couples to, with the biggest correction from the top quark (due to its large mass as given in Table 1.1). Figure 1.1a shows the loop correction of the Higgs' boson to the top quark. The evaluation of this Feynman diagram gives a mass term:

$$
\Delta m_{H}^{2}=-\frac{\left|\lambda_{f}\right|^{2}}{8 \pi^{2}} \Lambda_{\mathrm{UV}}^{2}+\ldots
$$

where $\lambda_{f}$ is the coupling strength of the top quark to the Higgs $(\sim 1)$, and $\Lambda_{\mathrm{UV}}$ is known as the ultra-violet cutoff, and represents the scale up to which the SM is valid, i.e. the Planck scale. The negative sign in Equation 1.1 is due to the fact that the top quark is a fermion, whose loop diagrams must have an overall negative sign arising from Fermi statistics [17]. It is clear that the correction $\Delta m_{H}$ to the Higgs' mass is much larger than the experimental result.

One simple solution is to cancel this mass term with an opposite contribution. 
This is possible if there exist two complex scalar particles with coupling strength to the Higgs, $\lambda_{s}=\left|\lambda_{f}\right|^{2}$. Figure 1.1b shows such a case, which has a correction to the Higgs' mass of:

$$
\Delta m_{H}^{2}=2 \times \frac{\lambda_{s}}{16 \pi^{2}} \Lambda_{\mathrm{UV}}^{2}+\ldots
$$

This cancellation comes naturally in supersymmetry (to be introduced in the next section), and it is one of the most attractive solutions to the hierarchy problem. The scalar in Figure 1.1b is the superpartner of the top quark, the stop.

\section{Dark Matter}

Measurements of the orbital velocities of spiral galaxies [18] give another hint that the SM may be incomplete. If galaxies are made up of all visible matter (and nearly massless neutrinos), and if the energy given off by the light is proportional to the mass of the galaxy, then it is predicted that the velocities of the stars decrease as a function of the distance away from the core of the galaxy. However, the observed data show a constant velocity in the disk of the galaxy. Figure 1.2 illustrates this discrepancy.

The most accepted solution to this problem is to postulate the existence of massive matter that is invisible, such that the overall mass of the galaxy is constant in the disk. This invisible matter is often referred to as the dark matter, and, as will be shown in the next section, supersymmetry offers excellent candidate particles for the dark matter. 

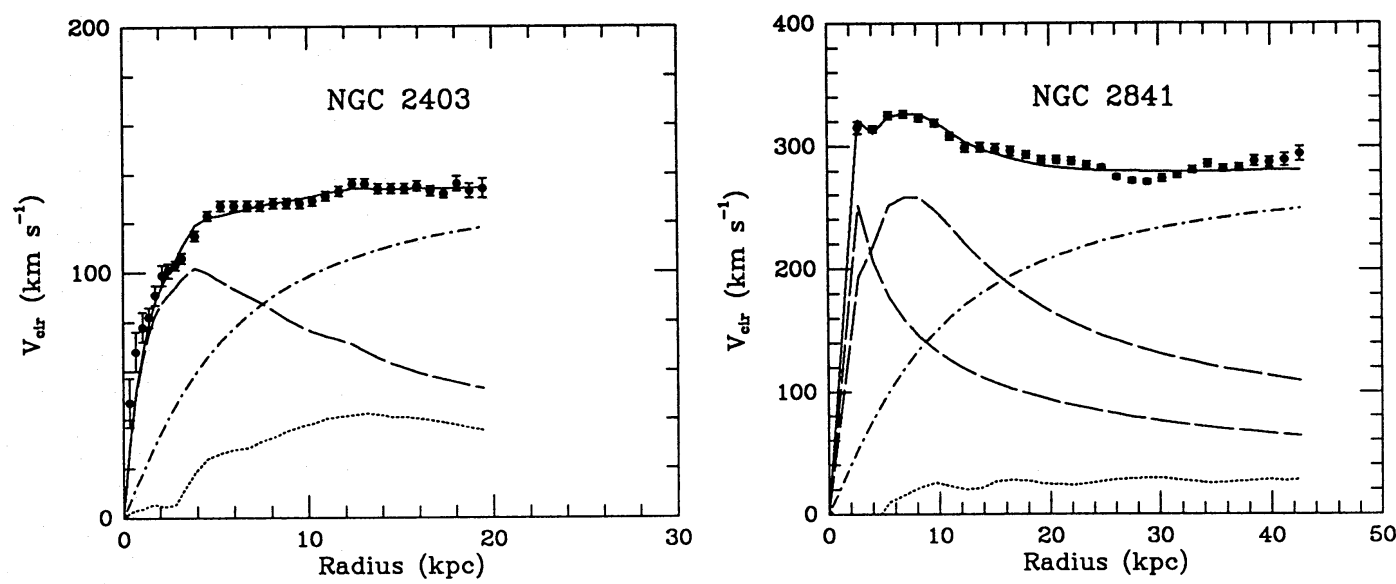

Figure 1.2: Rotation curves of example galaxies, NGC 2403 (left) and NGC 2841 (right), where the rotational velocity (y-axis) is plotted against the distance away from the centre of the galaxy ( $x$-axis). In each case, the observed rotational velocity is the sum of the expected velocities of visible stars (dashed line), gaseous matter (dotted line), and the dark matter halo (dash-dotted line). Figures from Reference [18].

\subsection{Supersymmetry}

\subsubsection{General Introduction}

Two examples of the limitations of the SM were given in the previous section. These can be addressed by extending or modifying the theory in what is called Beyond the Standard Model (BSM). There have been numerous attempts to formulate BSM theories in the past few decades, including the notion of the existence of extra dimensions [15]. Supersymmetry (SUSY) is one of these SM extensions which introduces a symmetry between fermions (half-integer spin particles) and bosons (integer spin particles) [14]. That is:

$$
Q \mid \text { fermion }>=\mid \text { boson }>, \quad Q \mid \text { boson }>=\mid \text { fermion }>
$$




\begin{tabular}{|c|c|c|c|c|c|c|}
\hline \multicolumn{2}{|c|}{ Names } & spin 0 & spin $1 / 2$ & Names & spin $1 / 2$ & spin 1 \\
\hline \hline squarks, quarks & $Q$ & $\left(\tilde{u}_{L}, \tilde{d}_{L}\right)$ & $\left(u_{L}, d_{L}\right)$ & gluino, gluon & $\tilde{g}$ & $g$ \\
$(\times 3$ families $)$ & $\bar{u}$ & $\tilde{u}_{R}^{*}$ & $u_{R}^{\dagger}$ & Winos, $W$ boson & $\tilde{W}^{ \pm}, \tilde{W}^{0}$ & $W^{ \pm}, W^{0}$ \\
& $\bar{d}$ & $\tilde{d}_{R}^{*}$ & $d_{R}^{\dagger}$ & Bino, $B$ boson & $\tilde{B}^{0}$ & $B^{0}$ \\
\hline sleptons, leptons & $L$ & $\left(\tilde{\nu}, \tilde{e}_{L}\right)$ & $\left(\nu, e_{L}\right)$ & & \\
$(\times 3$ families $)$ & $\bar{e}$ & $\tilde{e}_{R}^{*}$ & $e_{R}^{\dagger}$ & & \\
\hline Higgs, higgsinos & $H_{u}$ & $\left(H_{u}^{+}, H_{u}^{0}\right)$ & $\left(\tilde{H}_{u}^{+}, \tilde{H}_{u}^{0}\right)$ & & \\
& $H_{d}$ & $\left(H_{d}^{0}, H_{d}^{-}\right)$ & $\left(\tilde{H}_{d}^{0}, \tilde{H}_{d}^{-}\right)$ & & & \\
& & &
\end{tabular}

Table 1.3: Chiral (left) and gauge (right) supermultiplets in MSSM. The superpartners of the SM particles differ in spin by a half-integer, and they are denoted with a tilde. The notation ${ }^{*}$ and ${ }^{\dagger}$ denotes complex conjugate and complex conjugate transpose, respectively.

where $Q$ represents the supersymmetry transformation operator. In SUSY, the particle states are called supermultiplets and contain both fermions and bosons which are superpartners of each other. This implies that for each of the SM particles listed in Tables 1.1 and 1.2, there exist superpartners with spin that differs by a half unit.

\subsubsection{Particle Content in the MSSM}

The simplest, phenomenologically viable SUSY model is called the Minimally Supersymmetric SM (MSSM) [19, 20]. As the name suggests, it is an extension of the SM with a bare minimum addition of superpartners (only the superpartners of the existing SM particles are included). Table 1.3 shows the particle content in the MSSM. Note that the table is organized in terms of symmetry groups instead of physical states, as was the case in Table 1.1 and 1.2.

The SM fermions (Table 1.1) are either left- or right-handed and they transform differently under gauge transformation, therefore they reside in chiral supermultiplets 
and must have their own scalar superpartner. These scalar superpartners are called sfermions (the "s" denotes the fact that they are scalar), and denoted with a tilde. In particular, the scalar quarks are called squarks, and the scalar leptons are called sleptons. The SM Higgs' boson also resides in a chiral supermultiplet. In the MSSM, there are two Higgs' doublets: one for up-type quarks, and another for down-type quarks and charged leptons. Without the second doublet, the theory suffers from theoretical difficulties. The mixing of these doublets results in five Higgs' bosons: three neutral $\left(h^{0}, H^{0}, A^{0}\right)$, and two charged $\left(H^{ \pm}\right)$(more detail is given in Section 3.1.3).

The SM gauge bosons (Table 1.2) reside in gauge supermultiplets. Their superpartners are called gauginos (with the "ino" suffix), and they are also denoted with a tilde. The EW symmetry breaking (detailed in Section 3.1.3) allows the EW gauginos (the winos and the bino) to mix, resulting in mass eigenstates called neutralinos (denoted as $\tilde{\chi}^{0}$ ) and charginos (denoted as $\tilde{\chi}^{ \pm}$). As it will be discussed later, the lightest neutralino is a stable SUSY particle (called the LSP) that is weakly interacting, and it is a prime candidate for the dark matter introduced in Section 1.1.2.

\subsubsection{Previous Searches for SUSY Signatures}

The SUSY production rates, compared to that of the SM particle production rates, are expected to be significantly lower (with production cross sections, $\sigma$, of $\sigma_{\mathrm{SM}} \sim 10^{8} \mathrm{nb}$ and $\sigma_{\mathrm{SUSY}} \sim \mathcal{O}(1-10 \mathrm{pb})$, compared to $\sigma_{\text {Higgs }} \sim$ few $\left.10 \mathrm{pb}\right)^{2}$. In addition, most SUSY particles decay at the interaction point, therefore it is impossible to directly observe them in an experiment. Instead, these SUSY particles decay to SM particles

\footnotetext{
${ }^{2} 1$ barn $=1 \mathrm{~b}=10^{-28} \mathrm{~m}^{2}$
} 
which often leave traces in a detector that could be identified.

Previous searches for SUSY [21, 22] were carried out at the Large ElectronPositron collider (LEP) located at CERN [23], and at the Tevatron accelerator located at Fermilab [24]. LEP collected electron-positron collision data at the centre-of-mass energy near the $Z$-boson resonance (91 GeV) between 1989 and 1995, corresponding

to an integrated luminosity of $208 \mathrm{pb}^{-1}$, and over the $W^{ \pm}$pair threshold energies up to $208 \mathrm{GeV}$ between 1996 and 2000, corresponding to an integrated luminosity of $800 \mathrm{pb}^{-1}[25]$. Tevatron collected proton-antiproton collision data with centre-of-mass energy of $1.80 \mathrm{TeV}$ between 1992 and 1996 (Run-I), and $1.96 \mathrm{TeV}$ between 2001 and 2011 (Run-II). The corresponding integrated luminosity at the Tevatron is $12 \mathrm{fb}^{-1}$. Neither LEP nor Tevatron observed evidence for SUSY.

\subsection{Objective of the Thesis}

The main objective of this thesis work is to extend the efforts contributed by LEP and the Tevatron, and continue the search for possible SUSY signatures.

In order to experimentally observe the SUSY signatures, it is essential to have an excellent particle accelerator system capable of colliding high-energy particles at a high rate in order to produce these SUSY particles. The LHC [26] located at CERN is the current-generation particle accelerator that provides proton-proton collisions. It has already exceeded both the collision energies and the collision rates of the previous facilities, and therefore is capable of producing SUSY particles, if they exist. A particle detector with an accurate particle identification capability is also essential. The ATLAS detector [27] has excellent particle identification capability, and already collected sufficient collision data at high energy (integrated luminosity of $20.3 \mathrm{fb}^{-1}$ 
at the centre-of-mass energy of $\sqrt{s}=8 \mathrm{TeV}$ and $5 \mathrm{fb}^{-1}$ at $\sqrt{s}=7 \mathrm{TeV}$ ) for new, unprecedented results. Finally, a good scientific methodology is necessary to look for these signatures, and this is emphasized throughout the works presented in this thesis.

Since the idea of SUSY is so broad, the search criteria needs to be more focused. In this thesis, a specific production mode that directly produces sleptons and gauginos, called the EW production, is selected. Typical signatures for this production mode include multiple SM leptons and missing energy resulting from weakly interacting particles such as neutrinos and the LSP. This thesis mainly focuses on the signatures with exclusively two leptons and missing energy, and this is made possible by specific methodology taken throughout the analysis.

The work presented in this thesis was published as a part of an analysis by the ATLAS collaboration [28]. In particular, my unique contributions were: signal region optimization study for SR- $W W$ (Chapter 5), plotting of the kinematic distributions in the signal regions (Section 6.2), statistical interpretation of results of pMSSM models (Section 6.5), and combination of results with the trilepton analysis (Section 6.6).

\subsection{Outline of the Thesis}

The rest of this thesis is organized into individual chapters. Chapter 2 introduces the LHC and the ATLAS detector, the facilities that hosted the analysis detailed throughout this thesis work. Chapter 3 details the relevant theoretical considerations of the particular SUSY signatures for this search. Chapter 4 outlines the methodology and the definitions of the terminologies used throughout the analysis. Chapter 5 describes the process of defining a SUSY signal region and the signal optimization 
procedure, a part of the published analysis that I was responsible for. Chapter 6 presents the selected final results from the analysis and the exclusions of particular SUSY models, another part of my involvement in the publication. Finally, Chapter 7 summarizes this thesis work.

A summary of my personal contribution to the ATLAS Collaboration, a list of presentations given during my Ph.D. degree, and a list of acronyms are given at the end as appendices. 


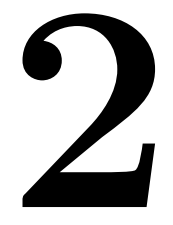

\section{The ATLAS Detector aT The $\mathrm{LHC}$}

In Chapter 1, a list of SM particles and the theorized SUSY particles are introduced. Some of them are unstable or exotic particles with large mass, and must be created in a laboratory setting. High energy particle collisions will allow these exotic particles to be produced by converting energy into mass (i.e. $E=m c^{2}$ ). The production rates of these particles are also very low, so in turn these collisions must occur frequently. The LHC is the facility best suited for this purpose, and it is described in Section 2.1. The unstable particles produced by the collisions decay immediately to stable ones, and they travel away from the interaction point. A particle detector is situated where the collisions take place, and it is designed to identify and record the passage of these stable particles. The stable particles interact with the detector materials and this allows the detector to identify them. The ATLAS detector is optimized for this purpose, and it is introduced in Section 2.2. A summary of the data collected and used for this thesis work is given at the end of the chapter. 


\section{$2.1 \quad$ The LHC}

The LHC (Large Hadron Collider) [26] is a high energy particle accelerator and collider, located at CERN (Conseil Européenne pour la Recherche Nucléaire), near Geneva, Switzerland. It is situated $100 \mathrm{~m}$ underground and consists of two $27 \mathrm{~km}$ rings of superconducting magnets. It is designed primarily as a proton-proton $(p p)$ collider, with the capability to accelerate two proton beams (each of the proton beams consists of 2,808 "bunches" with approximately $10^{11}$ protons) to centre-ofmass energy up to $14 \mathrm{TeV}$, and collide the beams at the centre of the detectors. The LHC uses electric fields created by radio-frequency cavities for acceleration, and superconducting magnets to bend the beams around the ring and also to direct the beams toward the centre of the detectors (the interaction points). Each of the bunches meet at the interaction point (called "bunch crossing") at 25 ns interval at the design luminosity of $10^{34} \mathrm{~cm}^{-2} \mathrm{~s}^{-1}$, and some of the protons within the bunch collide and interact with each other. Note that in 2010-2011 and 2012 the centre-of-mass energies were $7 \mathrm{TeV}$ and $8 \mathrm{TeV}$, respectively, with $50 \mathrm{~ns}$ bunch interval for both years. These high energy proton collisions allow precision measurements of SM parameters, of the newly discovered Higgs boson, and searches for new physics beyond the SM. In addition, the LHC can be used to accelerate lead ions $\left({ }^{208} \mathrm{~Pb}^{82+}\right)$ at the design energy of $2.76 \mathrm{TeV} /$ nucleon to create high-temperature states via either lead-lead or proton-lead collisions.

In order to produce beams with such energy at the LHC, CERN uses four stages of acceleration chains before reaching the LHC ring, as depicted in Figure 2.1. A linear accelerator (LINAC2) initiates the acceleration of protons, which are obtained 


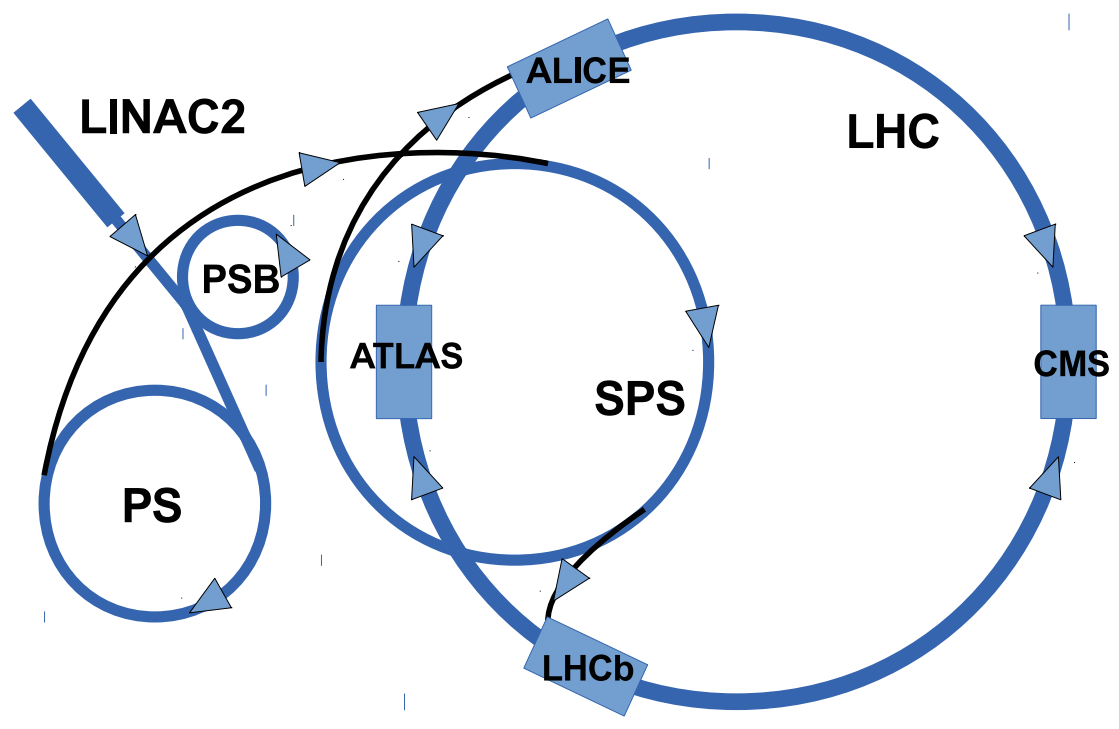

Figure 2.1: Injection chain at the LHC for proton beams. It is designed to accelerate each proton beam up to $7 \mathrm{TeV}$. The relative locations of the four main experiments are also shown. Figure based on Reference [26].

by stripping electrons from hydrogen atoms, up to an energy of $50 \mathrm{MeV}$. The Proton Synchrotron Booster (PSB) increases the beam energy to $1.4 \mathrm{GeV}$, which the Proton Synchrotron (PS) further accelerates to $25 \mathrm{GeV}$. The final stage of the acceleration before reaching the LHC is done by the Super Proton Synchrotron (SPS), which boosts the beam energy to $450 \mathrm{GeV}$, and finally into the main LHC ring.

The LHC houses four main experiments: ATLAS [27], ALICE [29], CMS [30], and LHCb [31]. ATLAS and CMS are general-purpose detectors designed to study SM and also physics beyond the SM. ALICE is optimized for heavy ion collisions (i.e. the lead ions as described earlier) to study quark-gluon plasma. LHCb is dedicated to the study of a charge-conjugation/parity $(\mathrm{CP})$ violation and rare decay events of heavy-flavour particles (i.e. the "bottom" quark). The work presented here involves only the ATLAS experiment with the $p p$ collisions. 


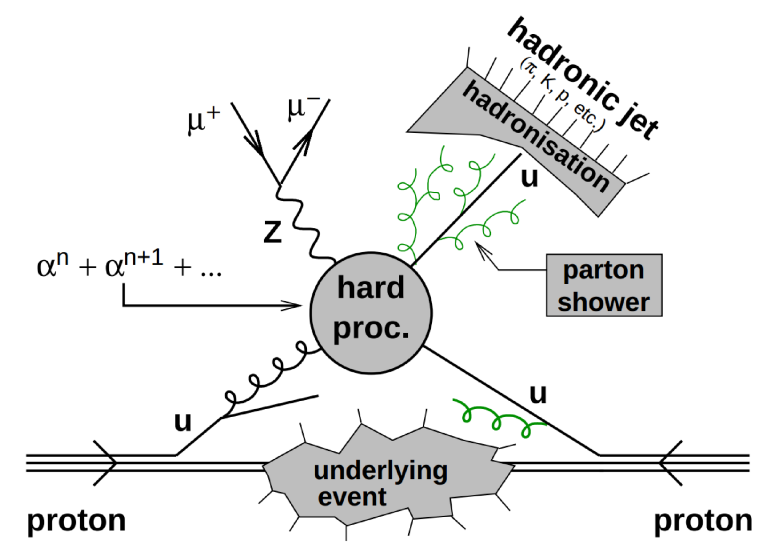

Figure 2.2: Schematic of an example pp collision process. A hard-scattering of a quark and a gluon leads to a final state involving a $Z$ boson and hadronic jet. Figure from Reference [32].

\subsection{1 $p p$ Collision Terminology}

\section{Hard-Scattering Events}

Protons that are accelerated and collided at the LHC are hadrons, and as such, each proton consists of three valence quarks (up, up, and down) and a sea of quarkantiquark pairs all bounded by the exchange of gluons. At high energy conditions, such as the case at the LHC where the protons travel at near-luminal speed, these constituent particles (collectively called partons) can be thought as being free.

A process of interest with high momentum transfer, known as a hard-scatter, is a result of the interaction of two partons, and the rest of the proton constituents may also interact in what is called an underlying event (UE). A free quark quickly transforms into a bounded state by a process known as hadronization, which then produces jets of particles, commonly referred to as hadronic jets. Figure 2.2 illustrates an example $p p$ collision.

\section{Luminosity}

Luminosity $L$ (with units of $m^{-2} s^{-1}$ or $b^{-1} s^{-1}$ ) is a quantity that relates the cross section $\sigma$ (with units of $m^{2}$ or $b$ ) of a physical process (in this case, $p p$ collisions) to 
the corresponding event rate $R$ (with units of $s^{-1}$ or $\mathrm{Hz}$ ):

$$
R=L \times \sigma,
$$

and $L$ is a process-independent quantity determined by the properties of the colliding beams [33]:

$$
L=F \times \frac{f \sum_{i} N_{1}^{i} N_{2}^{i}}{4 \pi \sigma_{x} \sigma_{y}},
$$

where $F=0.9$ is a factor arising from the non-zero crossing angle of the colliding beams, $f=11 \mathrm{kHz}$ is the beam-revolution frequency, $N_{1}^{i}$ and $N_{2}^{i}$ are number of protons in $i$-th bunch crossing, and $\sigma_{x}$ and $\sigma_{y}$ are the beam widths in $x$ and $y$, respectively.

From Equation 2.1, one can determine the number of expected events for a process, $\mathrm{N}$, by integrating over time:

$$
N=\int R d t=\sigma \int L d t
$$

where $\int L d t$ is referred to as integrated luminosity (with units of $m^{-2}$ or $b^{-1}$ ).

\section{Pile Up}

It is evident from Equation 2.3 that a higher luminosity results in higher event yield. Equation 2.2 states that a higher luminosity can be achieved by increasing the beam intensity, by increasing the frequency $f$ or reducing the beam widths $\sigma_{x}, \sigma_{y}$. This results in an increased chance of multiple proton collisions in the same bunch crossing, so-called pile-up events. Figure 2.3 shows a visualization of such an event, where it can be seen to become challenging to disentangle a hard-scattering process from the 


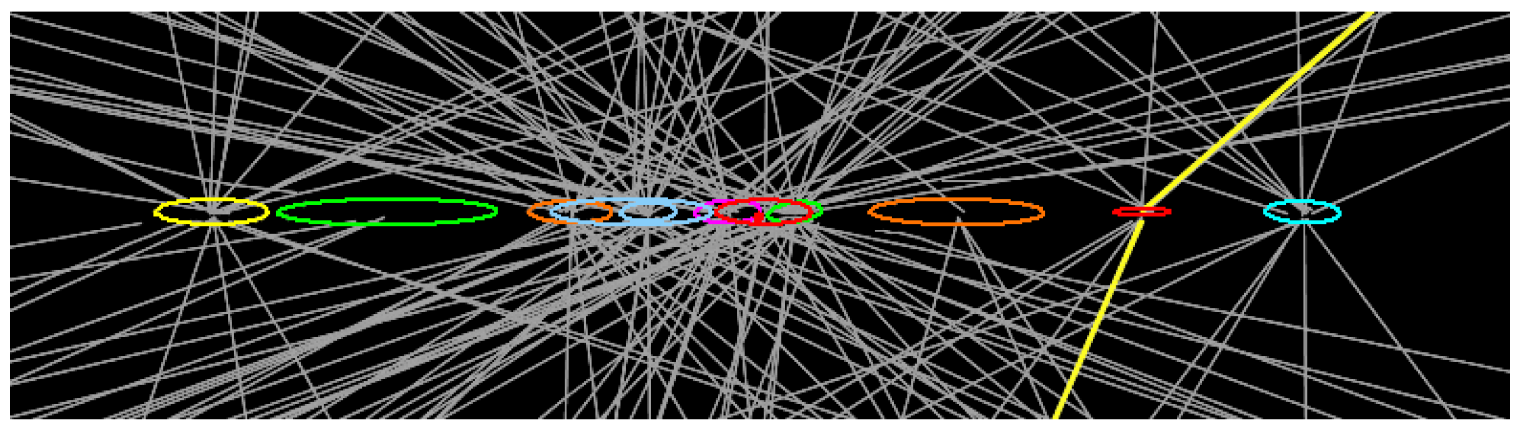

Figure 2.3: An example visualization of a pile-up event from 2011 ATLAS data run. Two proton bunches come from the left and right and individual protons interact in regions encircled. Particles then emerge from the collision, which are depicted by individual lines. In this example, A hard-scattered $Z$ boson decays to two muons (yellow tracks), while 10 other pile-up interactions are also reconstructed. Figure from Reference [34].

remaining pile-up events.

\subsection{The ATLAS Detector}

At the LHC, $p p$ collisions produce SM particles listed in Tables 1.1 and 1.2, as well as possible SUSY particles as described in Section 1.2.2. All of these particles interact differently with matter, and leave unique signatures depending on the type of materials they traverse. For this reason, typical particle detectors consist of several layers, as illustrated in Figure 2.4. By recognizing the signatures the particles leave in each layer, along with the position, energy and momentum measurements taken by each layer, the detector can identify the type of originating particle, and separate hard-scattering events from the pile-up events. Note that showers produced by particles in the calorimeter can "leak" into the next layer, thereby skewing the energy measurements. Thus, an MC simulation and calibration are always required to estimate the energy loss due to such possible effects. 


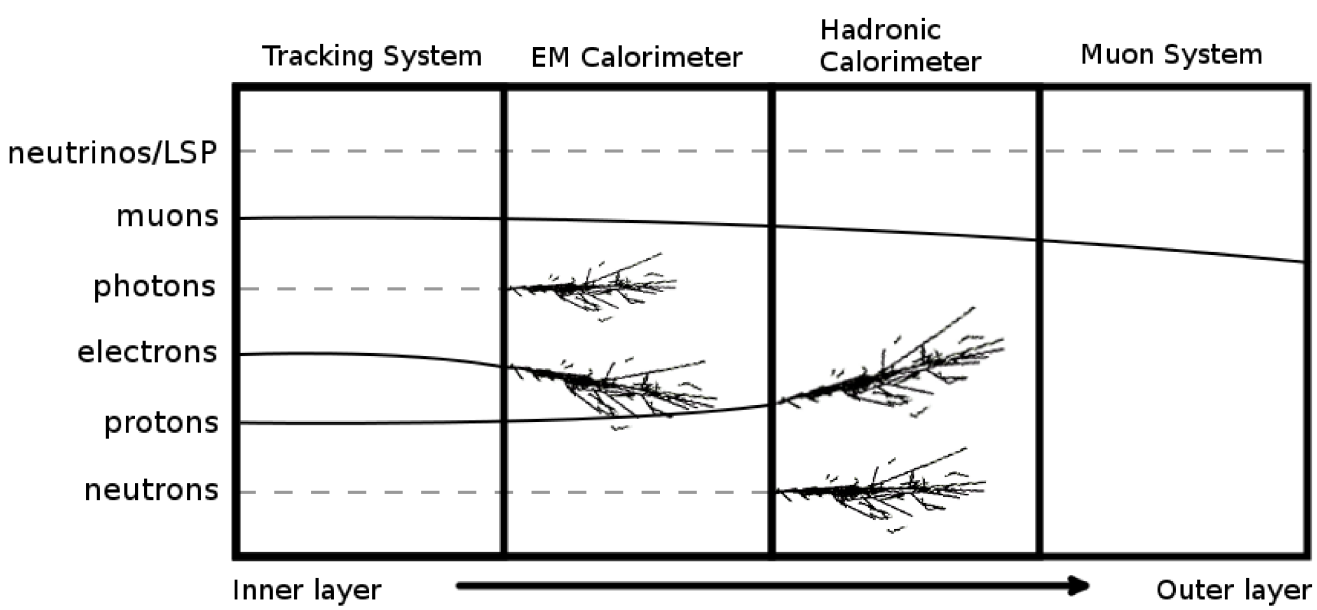

Figure 2.4: Schematic of particle paths in a particle detector with typical components. The interaction point is to the left, and the particles traverse from inner layer to the outer layer. Each type of particle leaves a signature in different layers of the detector. Dashed tracks are invisible to the detector.

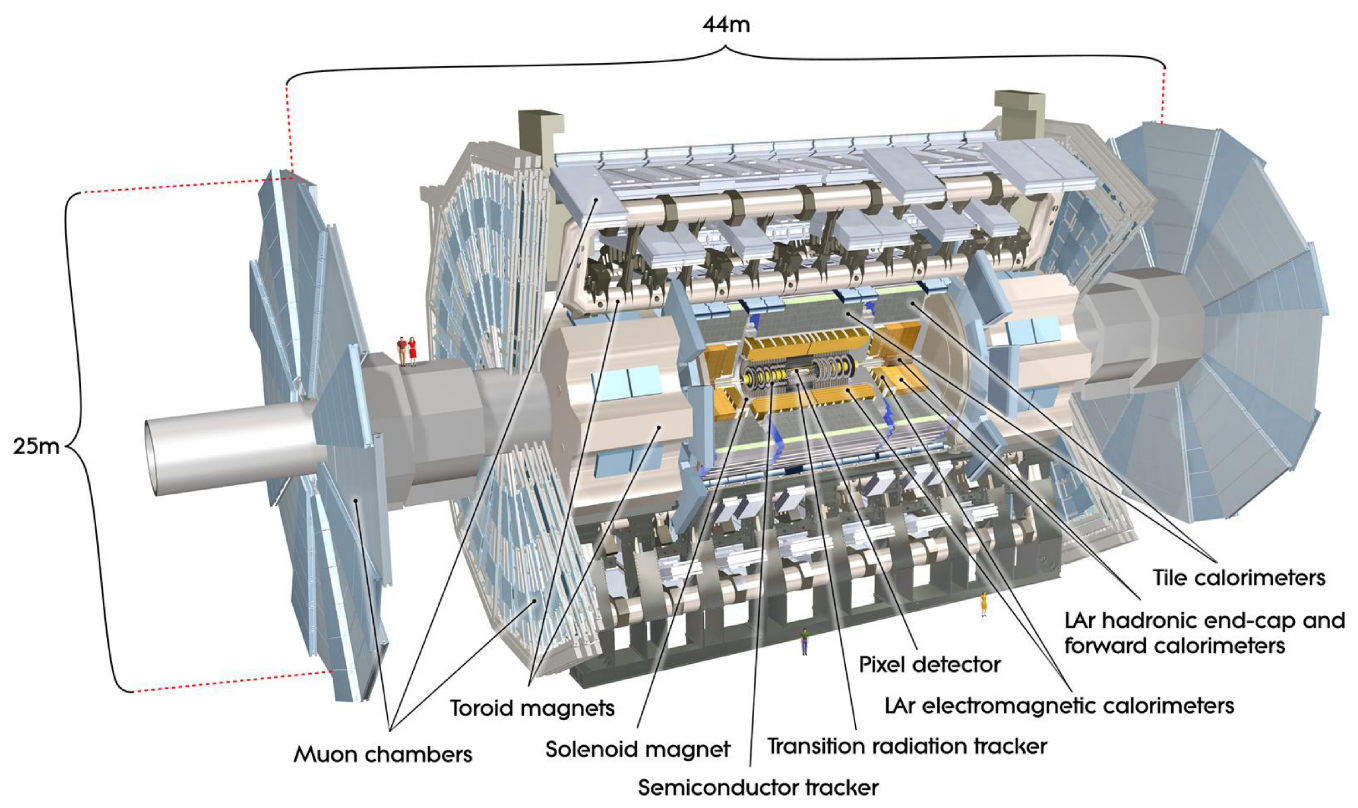

Figure 2.5: A cut-away view of the ATLAS detector showing the major components. Figure from Reference [27]. 
The ATLAS (A Toroidal LHC ApparatuS) detector, shown in Figure 2.5, possesses the above features of a typical particle detector. It has a forward-backward symmetric cylindrical geometry to take advantage of the conservation of momentum along the transverse plane, and has dimensions of $25 \mathrm{~m}$ in height and $44 \mathrm{~m}$ in length, and consists of approximately 7,000 tonnes of material. Due to the high energy of the beams and the luminosity of the LHC, the ATLAS detector features designs that accommodate the interaction rate and radiation dose from the $p p$ collisions provided by the LHC.

The coordinate system is defined such that the $z$-axis is aligned with the beam line, and the $x, y$ plane defines the transverse plane. The azimuthal angle $\phi$ is measured around the beam axis, and the polar angle $\theta$ is measured from the beam axis. It is, however, more common in collider physics to use a unit-less quantity called pseudorapidity $\eta$, in which the particle production is approximately constant and invariant under longitudinal boost. It is defined as:

$$
\eta=-\ln \left[\tan \left(\frac{\theta}{2}\right)\right] .
$$

A value of $\eta=0$ corresponds to $\theta=\pi / 2$ (perpendicular to the beam axis), and higher $\eta$ corresponds to a "forward" region (most of the detector has coverage of $|\eta|<5$, corresponding to $\theta \sim 0.013$ radians). A value of $\eta=\infty$ is along the beam axis, corresponding to $\theta=0$.

The ATLAS detector is comprised of four main components that work together to achieve sophisticated particle detection: the inner detector system, the calorimeter system, the muon system, and the trigger system. Aside from the main detector components, the offline software also plays an important role. Descriptions of these 

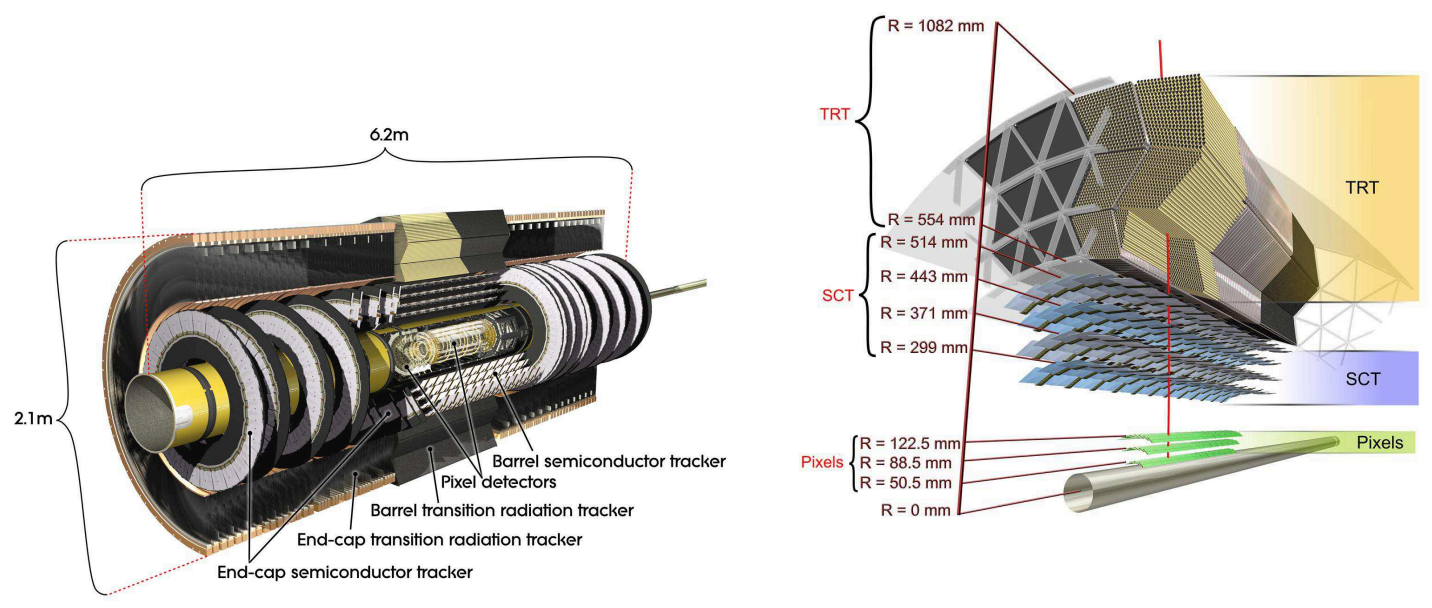

Figure 2.6: The overall layout of the ATLAS inner detector system. Figures from Reference [27].

components are summarized in the next section.

\subsubsection{Description of ATLAS Subdetectors}

\section{The Inner Detector System}

The inner detector system (ID) is the detector closest to the interaction point. Its primary role is to determine the momentum of charged particles by measuring their properties as they bend in a $2 \mathrm{~T}$ magnetic field produced by a central solenoid. It also provides vertexing for decaying particles and identification of pile-up and UE events. The ID covers the region $|\eta|<2.5$, and is comprised of three subcomponents: pixel detector, semiconductor tracker (SCT), and transition radiation tracker (TRT), as can be seen in Figure 2.6.

\section{The Calorimeter System}

The calorimeter system surrounds the ID, and it is responsible for the measurement 
of energy of particles and identification of activities originating from electromagnetic (EM) and hadronic particles. It also plays a crucial part in determining the escaped energy from a collision event (see Section 4.2.4). The layout of the calorimeter system is shown in Figure 2.7. The calorimeter system consists of three parts. The EM calorimeter is optimized for the detection of EM particles (i.e. electrons and photons) and consists of alternating layers of lead absorber and liquid argon (LAr) ionizing material, with coverage up to $|\eta|<3.2$ (barrel region covers $|\eta|<1.475$, and end-cap region covers $1.375<|\eta|<3.2$ ). The hadronic calorimeter detects hadronic showers (known as jets) and is a tile calorimeter consisting of steel absorbers and scintillating tiles (sampling calorimeter with alternating layers of copper and LAr) in the barrel (end-cap) region. The barrel region of the hadronic calorimeter covers $|\eta|<1.7$, while the end-cap region covers $1.5<|\eta|<3.2$. Finally, the forward calorimeter covers the region $3.1<|\eta|<4.9$, and utilizes alternating layer of copper (tungsten) absorbers and LAr for detection of EM particles (hadronic showers).

\section{The Muon System}

The ATLAS muon system is designed to detect muons and measure their momenta. It is located outside the calorimeter system, and its layout is shown in Figure 2.8. The toroid magnet system bends muon trajectories for precise track and momentum measurements in the range $|\eta|<2.7$. The Monitored Drift Tubes (MDT) are used for precision track reconstruction, and Cathode Strip Chambers (CSC) are used in the inner layer of the region $2.0<|\eta|<2.7$ in order to withstand the higher radiation flux in this region. The Resistive Plate Chamber (RPC) and Thin Gap Chamber (TGC) are used to trigger the muons in the barrel and end-cap region, defined by $|\eta|<1.05$ and $1.05<|\eta|<2.4$, respectively. 


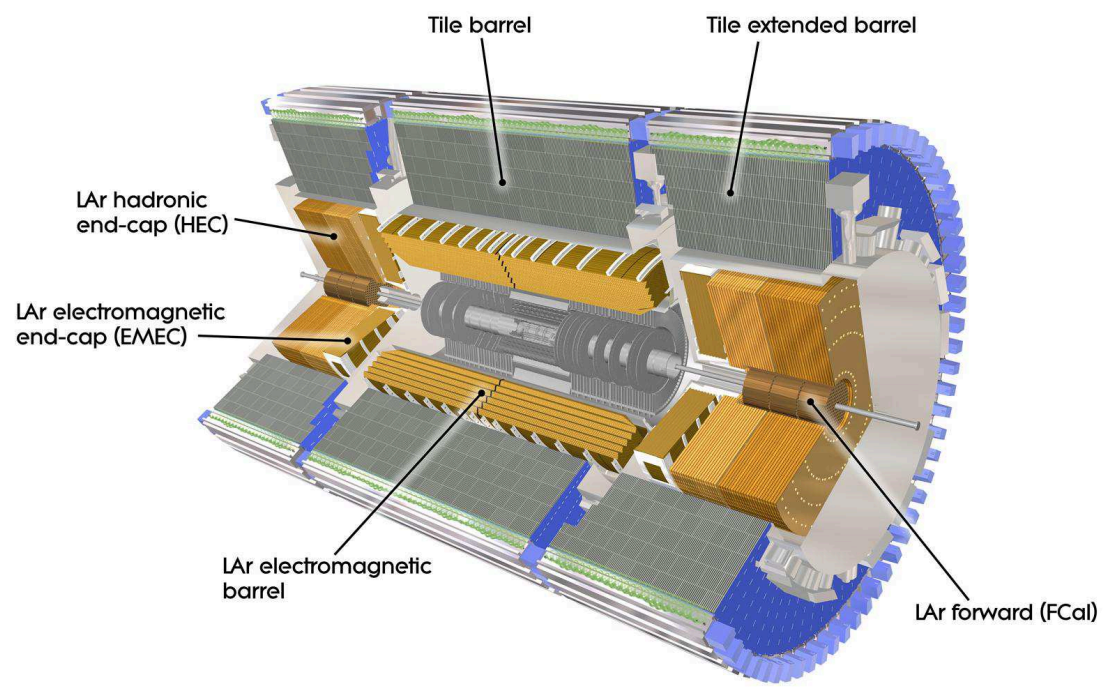

Figure 2.7: The overall layout of the ATLAS calorimeter system. Figure from Reference [27].

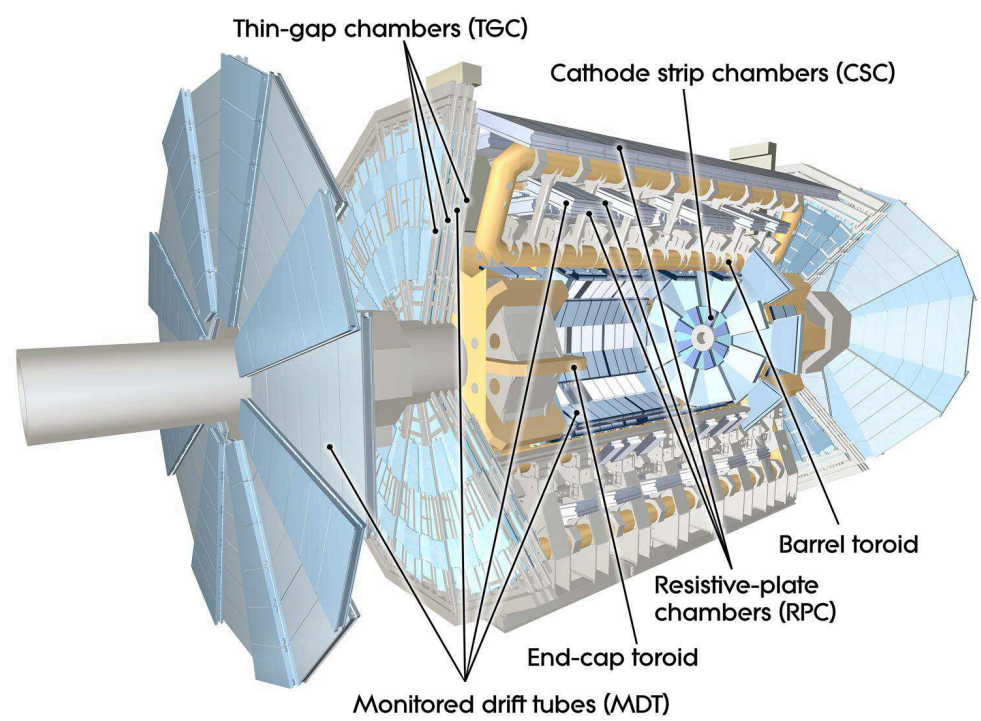

Figure 2.8: The overall layout of the ATLAS muon system. Figure from Reference [27].

\section{The Trigger System}

The LHC's $p p$ interaction rate at the design luminosity is approximately $1 \mathrm{GHz}$ (bunch 
crossing rate is $40 \mathrm{MHz}$, equivalent to the designed $25 \mathrm{~ns}$ bunch spacing), while the event data processing is limited to $400 \mathrm{~Hz}$. Most of the $p p$ collisions contain no interesting physics, so a trigger system needs to make a quick decision whether to keep or discard the collision event. ATLAS trigger system consists of three stages and together they are designed to achieve this quick decision-making: a hardwarebased level-1 (L1) system reduces the rate to $75 \mathrm{kHz}$ using calorimeter and muon spectrometer information, and makes the first decision within $2.5 \mu \mathrm{s}$; a software-based level-2 (L2) system reduces to $3.5 \mathrm{kHz}$ using full detector information (including the ID which is not used for L1) within a region of interest (ROI) defined by the L1 system, with the average processing time of $40 \mathrm{~ms}$; and the event filter (EF) system reduces the rate down to $400 \mathrm{~Hz}$ and classifies physics objects with the average processing time of a few seconds.

\section{The ATLAS Offline Software}

Once the events from the collisions pass all of the trigger criteria, data are stored for the offline analysis. The ATLAS offline software framework is called Athena, and its main purpose is calibration, event reconstruction, physics analysis, and production of Monte Carlo (MC) simulated events in conjunction with MC generators.

The Athena framework is also used to produce a user-analysis data format, called D3PD, which is a format readable by the CERN ROOT framework [35]. Athena takes the detector information for a given event to reconstruct the particle interactions and their kinematic properties into a D3PD. This D3PD format is used for this thesis work, and all of the plots are made using the ROOT framework. 

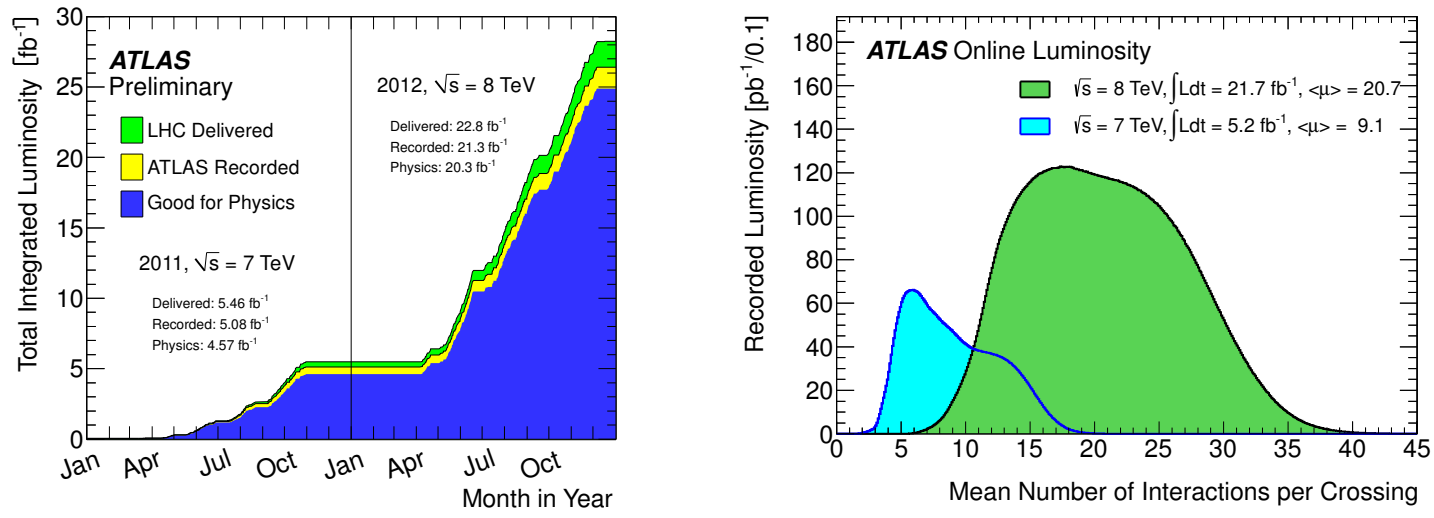

Figure 2.9: Integrated luminosity (left) and mean number of pp interactions per bunch crossing, equivalently the number of pile-ups (right) in 2011 and 2012 datataking periods. Figures from Reference [36]

\subsubsection{Collection of Data for Physics Analyses}

After a commissioning phase of the LHC, the initial $p p$ beam collisions took place in the fall of 2009, with the centre-of-mass energies $\sqrt{s}<3 \mathrm{TeV}(900 \mathrm{GeV}, 1.18 \mathrm{TeV}$, $2.36 \mathrm{TeV})$. In 2010, the centre-of-mass energy was increased to $\sqrt{s}=7 \mathrm{TeV}$, and roughly $35 \mathrm{pb}^{-1}$ of data were collected and used for first physics analyses.

In 2011, the luminosity of the LHC beam increased, allowing the accelerator to deliver $5.46 \mathrm{fb}^{-1}$ of integrated luminosity. However, due to inefficiencies and instabilities of the detector hardware, as well as data quality issues, only a subset of the data, corresponding to $4.57 \mathrm{fb}^{-1}$, was deemed suitable for physics analyses.

In 2012 , the energy of the proton beam was increased to $\sqrt{s}=8 \mathrm{TeV}$, and the LHC delivered $22.8 \mathrm{fb}^{-1}$ of data, of which $20.3 \mathrm{fb}^{-1}$ was suitable for physics analyses.

Figure 2.9 shows the integrated luminosity over time as well as the distribution of the average number of $p p$ interactions per bunch crossing $\langle\mu\rangle$ for the 2011 and 2012 data-taking periods. The values of $\langle\mu\rangle$ approximately correspond to the number of 


\begin{tabular}{c|c|c|c}
\hline Year & $\sqrt{s}(\mathrm{TeV})$ & $\int L d t$ (physics) & $\langle\mu\rangle$ \\
\hline 2009 & $0.9,1.18,2.36$ & $12 \mu \mathrm{b}^{-1}$ & $\sim 1$ \\
2010 & 7 & $35 \mathrm{pb}^{-1}$ & 2 \\
2011 & 7 & $4.57 \mathrm{fb}^{-1}$ & 9.1 \\
2012 & 8 & $20.3 \mathrm{fb}^{-1}$ & 20.7 \\
\hline
\end{tabular}

Table 2.1: Summary of collision energy, integrated luminosity, and the mean number of pp interactions per bunch crossing for ATLAS data runs since 2009.

primary vertices, or number of pile-ups, in an event (an example given in Figure 2.3 has 11 primary vertices). As it can be seen from Figure 2.9, the higher luminosity and centre-of-mass energy results in a busier collision environment, which in turn makes it more challenging to disentangle. Table 2.1 summarizes the relevant $p p$ collision information for ATLAS collision data since the data-taking started in 2009.

In this thesis work, only the $20.3 \mathrm{fb}^{-1}$ of physics data collected in the 2012 datataking period are used.

Currently, the LHC and the corresponding experiments (including ATLAS) are undergoing an upgrade phase with a goal to increase the centre-of-mass energy to $\sqrt{s}=13 \mathrm{TeV}$, and the data taking is projected to resume in early 2015 . 


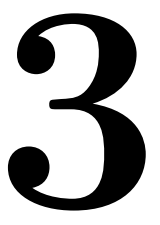

\section{INTRODUCTION TO ELECTROWEAK SUSY PRODUCTION}

In Section 1.2, a general overview of supersymmetry (SUSY) was presented. This chapter provides further details on the formalism of the theory, the general production of supersymmetric particles (called sparticles), the notion of final states and the typical observables at the LHC. In addition, two SUSY models that are relevant to this thesis work are introduced, and finally some Standard Model (SM) processes that mimic the SUSY signatures are detailed. This thesis focuses on electroweak (EW) SUSY processes.

\subsection{MSSM Theory}

\subsubsection{General SUSY Lagrangian}

As with the formulation of the SM, particle content and their interactions are governed by a series of Lagrangian densities $\mathcal{L}$ (also called the Lagrangian for simplicity) which 
are used to describe a quantity called the action:

$$
\mathcal{S}=\int \mathcal{L} d^{4} x
$$

According to Hamilton's principle [37, 38], the action should be invariant under a transformation by $\delta S$ (in this case, a SUSY transformation) that leads to the EulerLagrange equation of motion (where $\Psi$ represents a physical field) [17]:

$$
\partial_{\mu}\left(\frac{\partial \mathcal{L}}{\partial\left[\partial_{\mu} \Psi_{i}\right]}\right)-\frac{\partial \mathcal{L}}{\partial \Psi_{i}}=0
$$

In general, the SUSY Lagrangian is made up of several parts [14]. The simplest and minimal SUSY Lagrangian consists of the two-component Weyl fermion and the complex scalar fields, called chiral Lagrangian. The next simplest includes spin1 vector bosons, falling under gauge Lagrangian. Finally, there is the Lagrangian describing the gravity. This last part is outside the scope of this thesis work, therefore not discussed any further.

\section{Chiral SUSY Lagrangian}

Chiral supermultiplets (irreducible representation of sparticle states) were first introduced in Table 1.3 in terms of the MSSM, and they represent (s)quarks, (s)leptons, and Higgs(inos). In general, the free-interaction part of the chiral Lagrangian is written as [14]:

$$
\mathcal{L}^{\text {chiral }}=-\partial^{\mu} \phi^{* i} \partial_{\mu} \phi_{i}+i \psi^{\dagger i} \bar{\sigma}^{\mu} \partial_{\mu} \psi_{i}+F^{* i} F_{i}
$$

where the first term defines the boson content with the scalar boson field $\phi_{i}$, the second term defines the fermion content with the fermion field $\psi_{i}$ (and $\sigma^{\mu}$ is the Pauli 
matrix), and the last term is known as the auxiliary term. Without this last term, the action is invariant only for the on-shell case (only when the classical Euler-Lagrange equation of motion is imposed, resulting in the relation $\bar{\sigma}^{\mu} \partial_{\mu} \psi=0$ ), therefore does not hold quantum mechanically.

The interaction Lagrangian for chiral supermultiplets is written as (where c.c. means complex conjugate) [14]:

$$
\mathcal{L}_{\text {int }}^{\text {chiral }}=\left(-\frac{1}{2} W^{i j} \psi_{i} \psi_{j}-W^{i} W_{i}^{*}\right)+\text { c.c. }
$$

where $W^{i j}$ and $W^{i}$ are defined as:

$$
W^{i j}=\frac{\partial^{2} W}{\partial \phi_{i} \partial \phi_{j}}, \quad W^{i}=\frac{\partial W}{\partial \phi_{i}} .
$$

$W$ is called a superpotential, and it has the form:

$$
W=\frac{1}{2} M_{i j} \phi_{i} \phi_{j}+\frac{1}{6} y_{i j k} \phi_{i} \phi_{j} \phi_{k}
$$

where $M_{i j}$ is a symmetric mass matrix for the fermion fields, and $y_{i j k}$ is a Yukawa coupling of a scalar $\phi_{k}$ and two fermions $\psi_{i}$ and $\psi_{j}$.

\section{Gauge SUSY Lagrangian}

The gauge supermultiplet consists of a massless gauge boson field $A_{\mu}^{a}$, Weyl fermion gaugino field $\lambda^{a}$, and bosonic auxiliary field $D^{a}$ that is needed for the same reason as for the chiral auxiliary field $F_{i}$ introduced earlier. Its Lagrangian is written as [14]:

$$
\mathcal{L}^{\text {gauge }}=-\frac{1}{4} F_{\mu \nu}^{a} F^{a \mu \nu}+i \lambda^{\dagger a} \bar{\sigma}^{\mu} \nabla_{\mu} \lambda^{a}+\frac{1}{2} D^{a} D^{a},
$$


where $\nabla_{\mu}$ is the covariant derivative,

$$
F_{\mu \nu}^{a}=\partial_{\mu} A_{\nu}^{a}-\partial_{\nu} A_{\mu}^{a}+g f^{a b c} A_{\mu}^{b} A_{\nu}^{c}
$$

and $g$ and $f^{a b c}$ are the gauge coupling constant and gauge structure constant, respectively.

The gauge interaction Lagrangian consists of both chiral and gauge fields:

$$
\mathcal{L}_{\text {int }}^{\text {gauge }}=-\sqrt{2} g\left(\phi^{*} T^{a} \psi\right) \lambda^{a}-\sqrt{2} g \lambda^{\dagger a}\left(\psi^{\dagger} T^{a} \phi\right)+g\left(\phi^{*} T^{a} \phi\right) D^{a},
$$

where $T^{a}$ represents an $S U(n)$ group generator, which is a hermitian matrix satisfying $\left[T^{a}, T^{b}\right]=i f^{a b c} T^{c}$.

\section{Full SUSY Lagrangian}

For completeness, the full general SUSY Lagrangian is obtained by putting Equations $3.3,3.4,3.7$, and 3.9 together:

$$
\begin{aligned}
\mathcal{L}_{\text {SUSY }}= & \mathcal{L}^{\text {chiral }}+\mathcal{L}_{\text {int }}^{\text {chiral }}+\mathcal{L}^{\text {gauge }}+\mathcal{L}_{\text {int }}^{\text {gauge }} \\
= & -\nabla^{\mu} \phi^{* i} \nabla_{\mu} \phi_{i}+i \psi^{\dagger i} \bar{\sigma}^{\mu} \nabla_{\mu} \psi_{i}-\frac{1}{4} F_{\mu \nu}^{a} F^{a \mu \nu}+i \lambda^{\dagger a} \bar{\sigma}^{\mu} \nabla_{\mu} \lambda^{a} \\
& -\sqrt{2} g\left[\left(\phi^{*} T^{a} \psi\right) \lambda^{a}+\lambda^{\dagger a}\left(\psi^{\dagger} T^{a} \phi\right)\right]-\frac{1}{2}\left(W^{i j} \psi_{i} \psi_{j}+W_{i j}^{*} \psi^{\dagger i} \psi^{\dagger j}\right) \\
& +V\left(\phi, \phi^{*}\right),
\end{aligned}
$$

where the first line represents the free-interaction terms (with the $\partial_{\mu} \phi_{i}, \partial_{\mu} \psi_{i}$ in Equation 3.3 replaced with the covariant derivatives $\nabla_{\mu} \phi_{i}, \nabla_{\mu} \psi_{i}$ to be also gauge invariant), the second line represents the interaction terms, and the third line is the 
scalar potential:

$$
V\left(\phi, \phi^{*}\right)=F^{* i} F_{i}+\frac{1}{2} D^{a} D^{a}=W_{i}^{*} W^{i}+\frac{1}{2} g_{a}^{2}\left(\phi^{*} T^{a} \phi\right)^{2}
$$

where the auxiliary fields $F_{i}$ and $D^{a}$ have the form:

$$
F_{i}=-W_{i}^{*}, \quad D^{a}=-g\left(\phi^{*} T^{a} \phi\right) .
$$

\subsubsection{Soft Supersymmetry Breaking}

Supersymmetry in theory is an exact symmetry, but postulates sparticles that are degenerate in mass from the SM partners [39]. The non-observation of particles other than SM particles so far indicates that SUSY, if it exists, is broken at energy scale beyond the current experimental limits.

SUSY could be broken either explicitly or spontaneously. Spontaneous symmetry breaking occurs when a Lagrangian describing a system is invariant under a symmetry transformation but not at ground state. An example of this is breaking of a gauge symmetry known as the Higgs' mechanism [13]. On the other hand, explicit symmetry breaking occurs when a Lagrangian contains terms that is not invariant under a symmetry transformation. There is no consensus on how SUSY is broken, but there exist many models of spontaneous SUSY breaking scenarios. These always involve extensions of MSSM to include new particles or interactions (for example, introduction of gravity in minimal Supergravity, or mSUGRA, model), and they are

reviewed in Reference [14]. In MSSM, SUSY breaking is explicitly defined by a soft SUSY breaking term. 


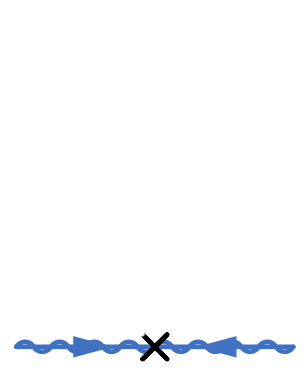

(a)
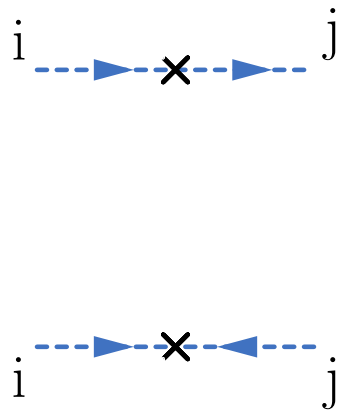

(b)

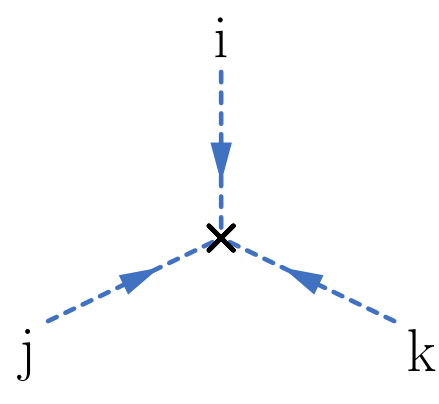

(c)

Figure 3.1: Soft SUSY-breaking terms: (a) gaugino mass $M_{a}$; (b) scalar squared mass $b^{i j}$ (top) and $\left(m^{2}\right)_{j}^{i}$ (bottom); and (c) scalar cubic coupling $a^{i j k}$. Figures based on Reference [14].

In general, the soft SUSY breaking terms can be included in the Lagrangian:

$$
\mathcal{L}_{\text {effective }}=\mathcal{L}_{S U S Y}+\mathcal{L}_{\text {soft }},
$$

where the term "soft" refers to the fact that the sparticle masses are in the phenomenologically acceptable range, between on the order of the $Z$ boson mass and the $\mathrm{TeV}$ scale. The soft Lagrangian term is written as:

$$
\mathcal{L}_{\text {soft }}=-\left(\frac{1}{2} M_{a} \lambda^{a} \lambda^{a}+\frac{1}{6} a^{i j k} \phi_{i} \phi_{j} \phi_{k}+\frac{1}{2} b^{i j} \phi_{i} \phi_{j}\right)+c . c-\left(m^{2}\right)_{j}^{i} \phi^{j *} \phi_{i},
$$

where $M_{a}$ is the gaugino mass term, $b^{i j}$ and $\left(m^{2}\right)_{j}^{i}$ are the scalar squared mass terms, and $a^{i j k}$ is the scalar cubic coupling, as diagrammatically illustrated in Figure 3.1. The above equation is said to break SUSY, because it involves only scalars and gauginos, but not their superpartners. 


\subsubsection{MSSM At Electroweak Scale}

The electroweak symmetry breaking $\left(S U(2)_{L} \times U(1)_{Y} \rightarrow U(1)_{\mathrm{EM}}\right)$ implies that the higgsinos and electroweak gauginos mix to form mass eigenstates: the neutral higgsinos $\left(\tilde{H}_{u}^{0}\right.$ and $\left.\tilde{H}_{d}^{0}\right)$ and the neutral gauginos $\left(\tilde{B}\right.$ and $\left.\tilde{W}^{0}\right)$ combine to form four neutralinos $\left(\tilde{\chi}_{1 . .4}^{0}\right)$; and the charged higgsinos $\left(\tilde{H}_{u}^{+}\right.$and $\left.\tilde{H}_{d}^{-}\right)$and charged winos $\left(\tilde{W}^{ \pm}\right)$ combine to form two charginos $\left(\tilde{\chi}_{1,2}^{ \pm}\right)$. By convention, the smaller index indicates smaller mass, and the lightest neutralino $\tilde{\chi}_{1}^{0}$ is assumed to be the lightest SUSY particle (LSP).

In the gauge-eigenstate basis $\psi^{0}=\left(\tilde{B}, \tilde{W}^{0}, \tilde{H}_{d}^{0}, \tilde{H}_{u}^{0}\right)$, the neutralino mass Lagrangian comes from the soft SUSY breaking term (i.e. from Equation 3.14):

$$
\mathcal{L}_{\tilde{\chi}^{0} \text { mass }}=-\frac{1}{2}\left(\psi^{0}\right)^{T} \mathbf{M}_{\tilde{\chi}^{0}} \psi^{0}+c . c,
$$

where

$$
\mathbf{M}_{\tilde{\chi}^{0}}=\left(\begin{array}{cccc}
M_{1} & 0 & -g^{\prime} v_{d} / \sqrt{2} & g^{\prime} v_{u} / \sqrt{2} \\
0 & M_{2} & g v_{d} / \sqrt{2} & -g v_{u} / \sqrt{2} \\
-g^{\prime} v_{d} / \sqrt{2} & g v_{d} / \sqrt{2} & 0 & -\mu \\
g^{\prime} v_{u} / \sqrt{2} & -g v_{u} / \sqrt{2} & -\mu & 0
\end{array}\right) .
$$

$M_{1}, M_{2}$, and $\mu$ are bino, wino, and higgsino mass terms, $g$ and $g^{\prime}$ are the gauge coupling constants for the $S U(2)$ and $U(1)$ groups, respectively, and $v_{u}$ and $v_{d}$ are vacuum expectation values (VEV) of $H_{u}^{0}$ and $H_{d}^{0}$, respectively.

The above mass matrix is diagonalized using a $4 \times 4$ unitary matrix $\mathbf{N}$ to obtain 
the mass eigenstates $\left(m_{\tilde{\chi}_{i}^{0}}\right)$ :

$$
\left(\mathbf{N}^{*} \mathbf{M}_{\tilde{\chi}^{0}} \mathbf{N}^{-1}\right)_{i}^{j}=\delta_{i}^{j} m_{\tilde{\chi}_{i}^{0}}
$$

In a similar fashion, the chargino mass Lagrangian in the gauge-eigenstate basis $\psi^{ \pm}=\left(\tilde{W}^{+}, \tilde{H}_{u}^{+}, \tilde{W}^{-}, \tilde{H}_{d}^{-}\right)$is:

$$
\mathcal{L}_{\tilde{\chi}^{ \pm} \text {mass }}=-\frac{1}{2}\left(\psi^{ \pm}\right)^{T} \mathbf{M}_{\tilde{\chi}^{ \pm}} \psi^{ \pm}+c . c
$$

where

$$
\mathbf{M}_{\tilde{\chi}^{ \pm}}=\left(\begin{array}{cc}
\mathbf{0} & \mathbf{X}^{\mathbf{T}} \\
\mathbf{X} & \mathbf{0}
\end{array}\right), \quad \mathbf{X}=\left(\begin{array}{cc}
M_{2} & g v_{u} \\
g v_{d} & \mu
\end{array}\right)
$$

Using two $2 \times 2$ unitary matrices, $\mathbf{U}$ for positively charged states $\left(\tilde{W}^{+}, \tilde{H}^{+}\right)$and $\mathbf{V}$ for negatively charged states $\left(\tilde{W}^{-}, \tilde{H}^{-}\right)$, the mixing matrix is diagonalized to obtain the mass eigenstates $\left(m_{\tilde{\chi}_{i}^{ \pm}}\right)$:

$$
\left(\mathbf{U}^{*} \mathbf{X} \mathbf{V}^{-1}\right)_{i j}=\delta_{i}^{j} m_{\tilde{\chi}_{i}^{ \pm}}
$$

The Higgs scalar potential in the MSSM can be obtained by rewriting the scalar potential (Equation 3.11) and the scalar squared mass terms in the soft SUSY breaking Lagrangian (Equation 3.14) in terms of MSSM Higgs doublets, $H_{u}=\left(H_{u}^{+}, H_{u}^{0}\right)$ and $H_{d}=\left(H_{d}^{0}, H_{d}^{-}\right)$:

$$
V_{\text {Higgs }}=V_{F-t e r m}+V_{D-t e r m}+V_{\text {soft }}^{m^{2}}+V_{\text {soft }}^{b},
$$


with

$$
\begin{aligned}
V_{F-\text { term }} & =|\mu|^{2}\left(\left|H_{u}^{0}\right|^{2}+\left|H_{u}^{+}\right|^{2}+\left|H_{d}^{0}\right|^{2}+\left|H_{d}^{-}\right|^{2}\right) \\
V_{D-\text { term }} & =\frac{1}{8}\left(g^{2}+g^{\prime 2}\right)\left(\left|H_{u}^{0}\right|^{2}+\left|H_{u}^{+}\right|^{2}-\left|H_{d}^{0}\right|^{2}+\left|H_{d}^{-}\right|^{2}\right)^{2}+\frac{1}{2} g^{2}\left|H_{u}^{+} H_{d}^{0 *}+H_{u}^{0} H_{d}^{-*}\right|^{2} \\
V_{\text {soft }}^{m^{2}} & =m_{H_{u}}^{2}\left(\left|H_{u}^{0}\right|^{2}+\left|H_{u}^{+}\right|^{2}\right)+m_{H_{d}}^{2}\left(\left|H_{d}^{0}\right|^{2}+\left|H_{d}^{-}\right|^{2}\right) \\
V_{\text {soft }}^{b} & =b\left(H_{u}^{+} H_{d}^{-}-H_{u}^{0} H_{d}^{0}\right)+\text { c.c. }
\end{aligned}
$$

Then, imposing electroweak symmetry breaking, $\left\langle H_{u}^{+}\right\rangle$can be set to 0 by choosing an appropriate gauge transformation. This implies $\left\langle H_{d}^{-}\right\rangle=0$ due to the requirement of $\partial V_{\text {Higgs }} / \partial H_{u}^{+}=0[14,40]$. The remaining two neutral Higgs VEV are related to the $Z$ boson mass as:

$$
v_{u}^{2}+v_{d}^{2}=2 m_{Z}^{2} /\left(g^{2}+g^{\prime 2}\right) \approx(174 / \mathrm{GeV})^{2}
$$

and the ratio of the $\mathrm{VEV}$ is commonly denoted as $\tan \beta \equiv v_{u} / v_{d}$.

SUSY requires two Higgs doublets in $S U(2)_{L}$ group, and therefore has eight real degrees of freedom, three of which become longitudinal modes of $W^{ \pm}$and $Z$ bosons via the Higgs' mechanism [41]. The remaining five mass eigenstates are three neutral states $\left(h^{0}, H^{0}, A^{0}\right)$ and two charged states $\left(H^{+}, H^{-}\right)[14]$.

\subsubsection{R-Parity}

R-parity is an arbitrary quantum number assigned to all elementary particles, relating baryon number, B, lepton number, L, and spin, S. It is defined as [14]:

$$
R=(-1)^{3(B-L)+2 S}
$$


All of the SM particles have $R=+1$ while sparticles have $R=-1$. In the MSSM, $\mathrm{R}$-parity is conserved, meaning that the product of final $\mathrm{R}$-parities matches with the initial $\left(R_{\text {initial }}=\Pi R_{\text {final }}\right)$. Without the requirement of this parity conservation, the theory violates B, L, as well as lepton flavour conservation which leads to inconsistencies with previous experimental data, notably the non-observation of proton decay [14]. The consequence of R-parity conservation is threefold: SUSY particles must be pair-produced at collider experiments since the initial beam has $R_{\text {initial }}=+1$; the produced sparticle will always decay to an odd number of LSP; and the LSP with $R=-1$ is absolutely stable and, if it is electrically neutral, this is a natural candidate for the dark matter.

\subsection{Production Process}

If MSSM with R-parity conservation is assumed, SUSY particles are produced in pairs from the hard-scattered parton collisions within the accelerated proton beams at the LHC. For EW processes, the direct pair-production of gauginos $\left(\tilde{\chi}^{ \pm}, \tilde{\chi}^{0}\right)$ and sleptons $\left(\tilde{\ell}^{ \pm}\right)$is possible, where the $s$-channel production dominates (see Figure 3.2a). For strong processes, the gluons and quarks from the incoming proton beams participate to pair-produce squarks and gluinos, as depicted in Figure 3.2b.

Figure 3.3 shows the cross section of sparticle pair-production at the LHC with a centre-of-mass energy of $\sqrt{s}=8 \mathrm{TeV}$ as a function of their average mass. It can be seen that the QCD production ( $\tilde{q} \tilde{q}$ and $\tilde{g} \tilde{g}$ pair-production) has a much higher production cross section than the EW process, meaning that they are more abundantly produced. However, with the early running of the LHC, there has been no observation of SUSY-like events, leading to a strong limit on squark and gluino 


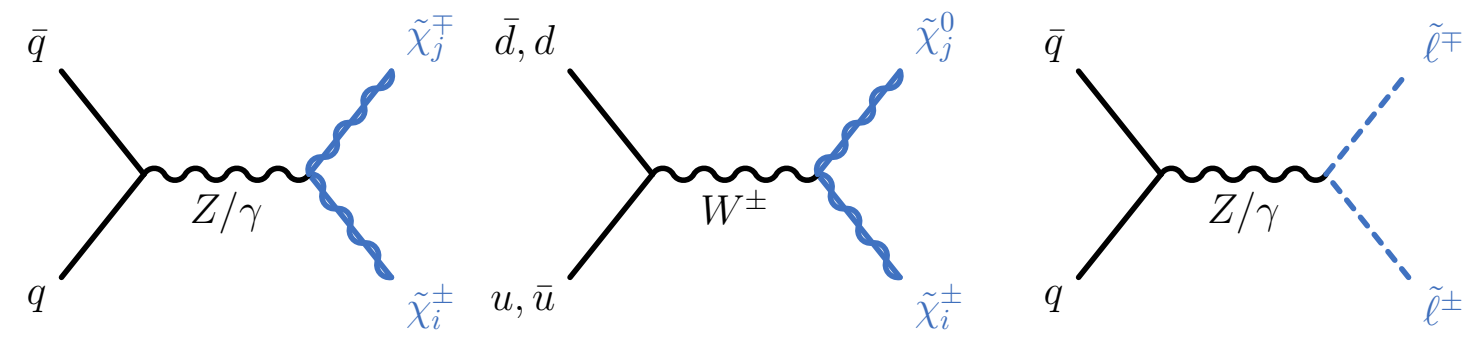

(a)
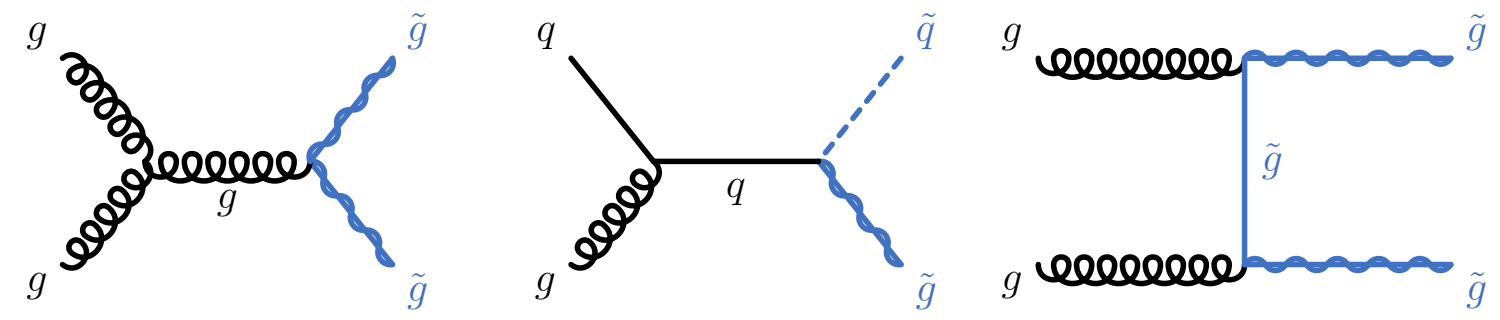

(b)

Figure 3.2: Example production processes of sparticles at the $L H C$ at (a) the EW scale and (b) the QCD scale. For a complete list of SUSY production modes, see Reference [14].

masses of order $1 \mathrm{TeV}$ [43]. In this scenario, EW sparticle production (i.e. direct gaugino or slepton pair-production) is more likely at the LHC, and hence it is the main focus of this thesis.

\subsection{Final States}

All SUSY particles except for the LSP are expected to be unstable, and so undergo decay chains at the interaction point (even before reaching the ATLAS detector). A final state refers to a state where all of the unstable particles have decayed to a stable state. These decays involve SM particles that the ATLAS detector is capable of 


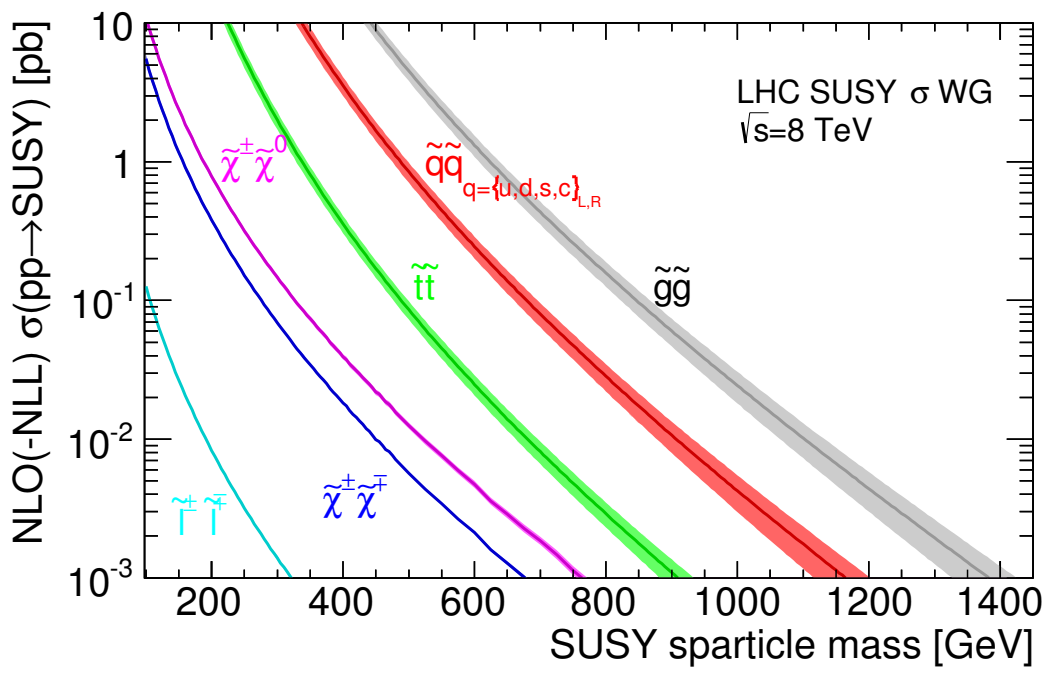

Figure 3.3: Production cross sections of sparticles as a function of the average mass of produced sparticles. Figure based on Reference [42].

identifying. The pair-produced sparticles as depicted in Figure 3.2 can form distinct final states depending on the decay processes involved.

Charginos and neutralinos decay via EW processes, usually involving leptons or gauge bosons. Following the Lagrangians for gauge multiplets in Equation 3.7 and Equation 3.9, Feynman diagrams can be drawn for EW interactions, as presented in Figure 3.4. If sleptons are light (which is required by so-called natural SUSY theories that predict the sparticles to be detectable at the $\mathrm{LHC}$ scale, i.e. below $\mathcal{O}(1 \mathrm{TeV}))$ [44], the sleptons almost always decay to a lepton and a LSP (i.e. $\left.\tilde{\ell} \rightarrow \ell \tilde{\chi}_{1}^{0}\right)$.

Combining diagrams from Figures 3.2 and 3.4 will form the complete picture of the physical processes for expected EW SUSY interaction at the LHC. In particular, Figure 3.5 shows the processes with two final state leptons (so-called dilepton final states), where the hashed circle represents the combination of possible EW processes from Figure 3.2a. In all cases, due to R-parity conservation, the final state involves 

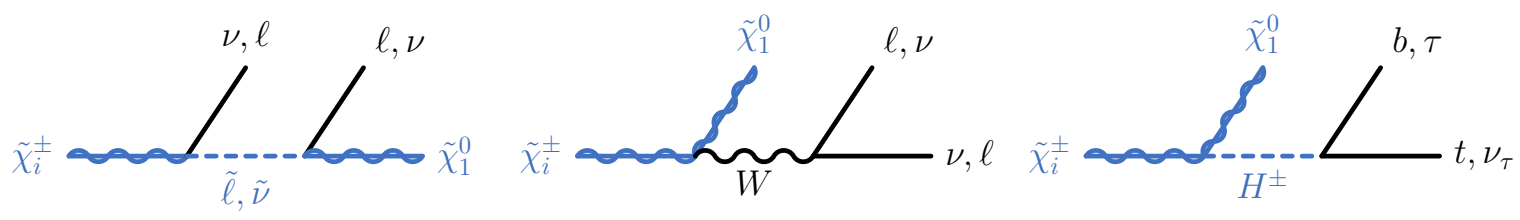

(a)
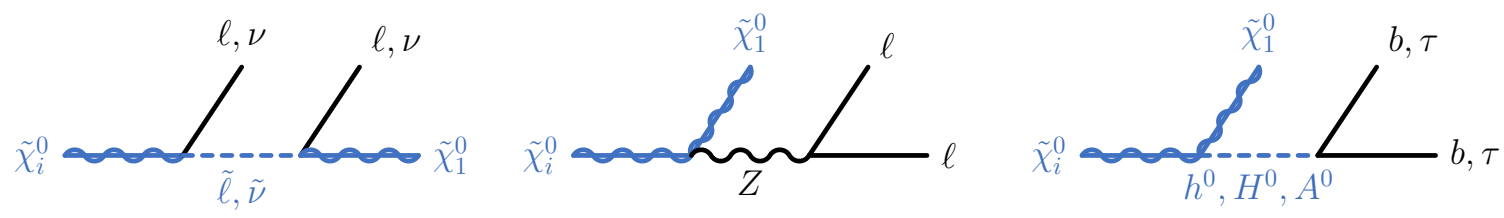

(b)

Figure 3.4: Decay chains of (a) charginos and (b) neutralinos. Figures based on Reference [14].

LSP that are stable and non-interacting. As such, they leave the detector unnoticed, and create an imbalance in momentum sum in the transverse plane (also known as missing energy - further discussion can be found in Section 4.2.4). Neutrinos are weakly interacting SM particles, and so they also produce extra missing energy. In some cases, quarks are involved in the final state (for example Figure 3.5d). The quarks hadronize and then interact with detector material, producing hadronic activity in the detector and leaving a signature known as a jet.

Depending on the topology of the events, the strategy to identify the possible signal SUSY events or SM events that mimic the SUSY signal may also be different. For example in Figure 3.5d, the fact that both leptons originate from a $Z$ boson can be exploited to select appropriate candidate signal events. For this thesis work, the leptons refer to either electrons $e$ or muons $\mu$. The identifications of tau leptons require special treatment, and therefore are not considered. Its analysis can be found in Reference [45]. 


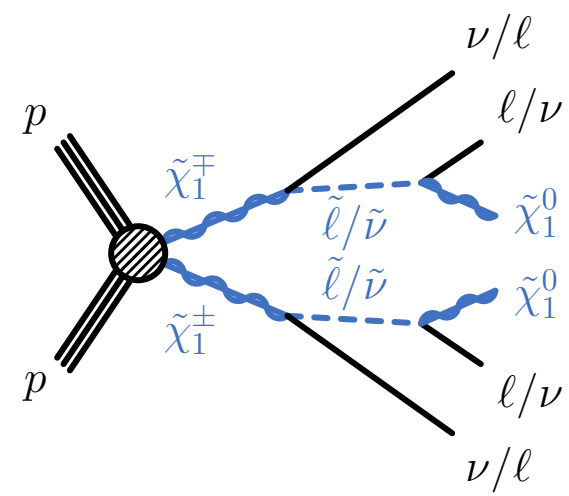

(a)

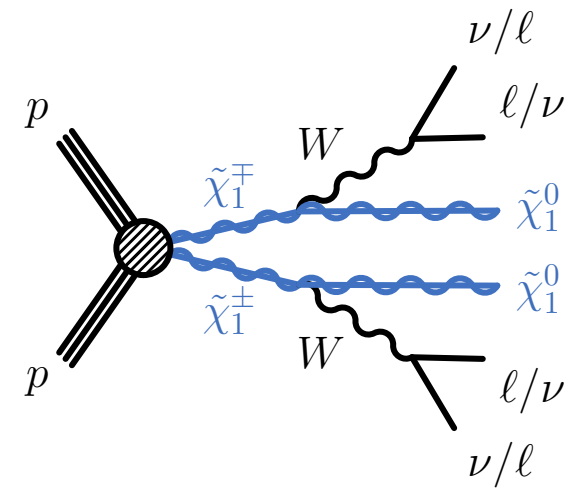

(c)

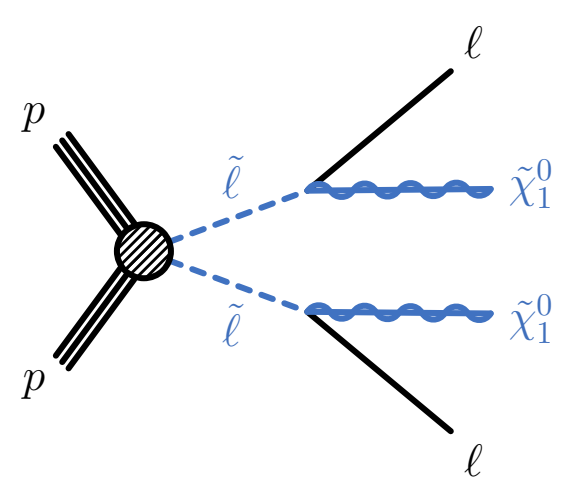

(b)

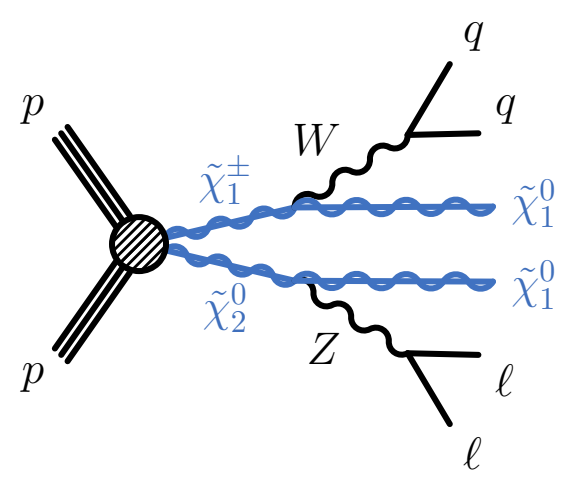

(d)

Figure 3.5: Signal topology for EW SUSY production mode resulting in a final state with two leptons and a missing energy.

\subsection{SUSY Signal Models}

For this thesis work, two different SUSY models are considered: phenomenological MSSM (pMSSM) for the interpretation of the results (detailed in Chapter 6); and Simplified Model for the optimization study of the signal regions (detailed in Chap- 
ter 5). The details of these two models are given in the following subsections.

\subsection{1 pMSSM}

The MSSM introduces over 100 new parameters to the SM, most of which arise from soft SUSY breaking terms from Section 3.1.2. However, the number of parameters that are relevant to the phenomenology at the LHC is much smaller. These free parameters could be reduced by assuming charge-parity (CP) conservation. This will eliminate all the complex phases resulting in $\mathrm{CP}$ violation analogous to the SM. Another possible simplification is to eliminate trilinear couplings and Yukawa couplings for 1st and 2nd generation sfermions, and assume these sfermions have degenerate masses. These simplifications lead to the pMSSM [46].

The remaining 19 parameters are manageable enough to be systematically searched for at the LHC. They are:

- 6 squark mass parameters: $M_{x, i i}$, where $x=Q, u, d$ and the index $i$ represents the $i$ th squark generation, with the first two generations being mass-degenerate

- 4 slepton mass parameters: $M_{x, i i}$, where $x=L, e$ and the index $i$ represents the $i$ th slepton generation, with the first two generations being mass-degenerate

- 3 gaugino mass parameters: $M_{1}, M_{2}$, and $M_{3}$

- 3 trilinear couplings: $A_{t}, A_{b}$, and $A_{\tau}$

- 3 Higgs(-ino) parameters: $\mu, m_{A}$, and $\tan \beta$

Within the pMSSM, two separate types are considered, and for each type, additional assumptions are enforced.

\section{Direct gaugino production with decays involving sleptons}

This corresponds to Figure 3.5a, where only the right-handed sleptons are included. 
The masses of the sleptons are assumed to be $\frac{1}{2}\left(m_{\tilde{\chi}_{1}^{ \pm}}+m_{\tilde{\chi}_{2}^{ \pm}}\right)$and the masses of squarks are set to $2 \mathrm{TeV}$ to suppress their contributions. The value of $\tan \beta$ is set to 6 . This low value ensures a good mix of selectrons, smuons, and staus. The value of $m_{A}$ is set to $500 \mathrm{GeV}$, and only the stop trilinear coupling is non-zero. These assumptions are put in place by the SUSY working group within the ATLAS collaboration, and they ensure that the lightest higgs mass $h^{0}$ to be in the range of newly discovered boson [1].

\section{Direct gaugino production with decays with no sleptons}

This corresponds to Figures 3.5c/d. For this type, the parameter choices are the same as above, except that the masses of the sleptons are also set to $2 \mathrm{TeV}$ to suppress their contributions. The value of $\tan \beta$ is also different, and is set to 10 .

\subsubsection{Simplified Model}

The Simplified Model has minimal particle content to produce SUSY-like events, and parameterized by sparticle masses and their branching ratios. For each of the diagrams considered in Figure 3.5, the masses of all sparticles other than those appearing in the diagram are set to $\mathcal{O}(100 \mathrm{TeV})$ to suppress any contribution. Both $\tilde{\chi}^{0}$ and $\tilde{\chi}^{ \pm}$are assumed to be purely wino-like except for direct slepton production case (remember that gauginos are mixtures of gauge superfields).

For each of the topologies in Figure 3.5, additional assumptions are enforced.

\section{$\tilde{\chi}_{1}^{ \pm} \tilde{\chi}_{1}^{ \pm}$Production with intermediate $\tilde{\ell}$ (Figure $3.5 \mathrm{a}$ )}

This assumes that each $\tilde{\chi}_{1}^{ \pm}$decays via sleptons or sneutrinos with a branching ratio of $50 \%$. The slepton/sneutrino masses are degenerate and have values of $\frac{1}{2}\left(m_{\tilde{\chi}_{1}^{ \pm}}+m_{\tilde{\chi}_{1}^{0}}\right)$. 
The lowest mass splitting between $\tilde{\chi}_{1}^{ \pm}$and $\tilde{\chi}_{1}^{0}$ is $35 \mathrm{GeV}$.

$\tilde{\ell} \tilde{\ell}$ Production (Figure 3.5b)

The masses of all sparticles except for sleptons (excluding staus) and LSP are set to 2.5 TeV to suppress any other contributions than the diagram of interest. The LSP is assumed to be purely bino-like. The sleptons can be left- or right-handed, and they are assumed to be mass-degenerate.

\section{$\tilde{\chi}_{1}^{ \pm} \tilde{\chi}_{1}^{ \pm}$Production with no intermediate $\tilde{\ell}$ (Figure $3.5 \mathrm{c}$ )}

This assumes that $\tilde{\chi}_{1}^{ \pm}$decay to $W$ boson has a $100 \%$ branching ratio, and consider only the leptonic decays of the $W$ bosons. The mass splitting between $\tilde{\chi}_{1}^{ \pm}$and $\tilde{\chi}_{1}^{0}$ larger than the $W$ boson mass is assumed.

$\tilde{\chi}_{1}^{ \pm} \tilde{\chi}_{2}^{0}$ Production with intermediate $W$ and $Z$ bosons (Figure $3.5 \mathrm{~d}$ )

This assumes that $\tilde{\chi}_{1}^{ \pm}$decay to $W$ boson and $\tilde{\chi}_{2}^{0}$ decay to $Z$ boson have $100 \%$ branching ratios by setting the slepton/sneutrino mass to be high. It considers only the leptonic decays of the $Z$ bosons, and only the cases with hadronic decays of the $W$ bosons are examined. The mass splitting between the $\tilde{\chi}_{1}^{ \pm}$and $\tilde{\chi}_{1}^{0}$ and between the $\tilde{\chi}_{2}^{0}$ and $\tilde{\chi}_{1}^{0}$ are assumed to be larger than $50 \mathrm{GeV}$ and $12.5 \mathrm{GeV}$, respectively.

\subsection{Standard Model Backgrounds}

The ATLAS detector can identify visible final states, but not the intermediate states or non-interacting particles, such as the LSP. For each of the signal topologies in Figure 3.5, there exist SM processes with identical or similar final states, but with different intermediate states (without SUSY processes). These SM events will mimic 

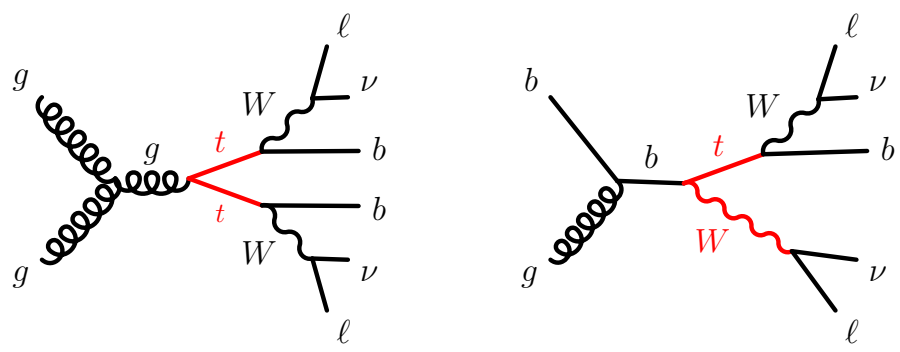

Figure 3.6: Examples of SM top production. Left is the top pair production via gluon fusion. Right is the associated single-top production with the $W$ boson. The particles that characterize this SM background are highlighted in red.

SUSY events and they could be misidentified as SUSY events (which would imply new physics), although in reality they have no new physical implications. These events are called backgrounds, and the ability to identify and reject these events is important.

Of the SM backgrounds presented below, the top, the WW, and ZV backgrounds are the dominant ones for the dilepton EW SUSY signatures.

\subsubsection{Top}

Top backgrounds refer to either top pair-production $(t \bar{t})$ or associated single-top production with the $W$ boson $(W t)$. The dominant production mode and the final states involving two leptons can be seen in Figure 3.6. The similarity in final states between the SM top and SUSY events can be seen by comparing with Figure 3.5.

The top quark decays to a $W$ boson and a $b$ quark with $91 \%$ probability [3]. The unique behaviour of the $b$ quark, in particular the fact that the B hadrons are stable enough to produce a displaced vertex that is measurable with a detector, could be used to identify the top backgrounds. 

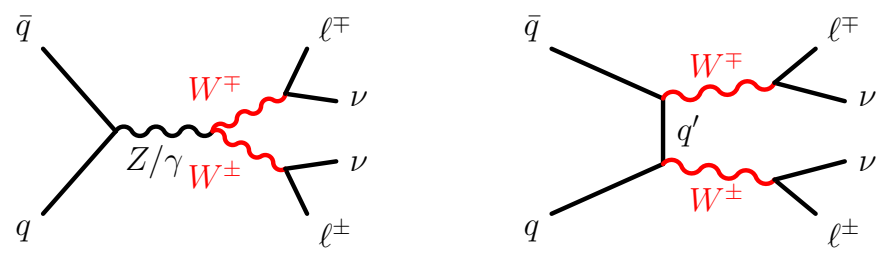

Figure 3.7: Examples of $S M W W$ production. Left is the s-channel process. Right is the t-channel process. The $W$ bosons that characterize this SM background are highlighted in red.

\subsubsection{WW}

SM $W W$ production is another form of SM events that has a very similar final state to the expected signal, as it can be seen from Figure 3.7. The only difference in the final state between Figure 3.7 and Figure 3.5c, for example, is the LSP, which is invisible to the ATLAS detector, and depending on the direction of the LSP, the missing energy contribution could either add or cancel. Because of this, the $W W$ background is more difficult to separate from the signal SUSY events.

\subsubsection{ZV $(\mathrm{ZZ} / \mathrm{ZW})$}

SM $Z V$ (where $V$ is a vector boson, i.e. either the $W$ or $Z$ boson) events are similar to the $W W$ process, but involve $Z$ bosons. They could also mimic signal SUSY events as it can be seen in Figure 3.8. As an example, one of the $Z$ bosons produces two hard leptons with enough momentum to be triggered (see Section 4.1.2 for the $p_{\mathrm{T}}$ threshold), while the other $Z$ or $W$ boson could produce soft jets or soft leptons with $p_{\mathrm{T}}<10 \mathrm{GeV}$ and therefore unidentified by the detector.

If both leptons come from the $Z$ boson, their invariant mass should be the $Z$ 

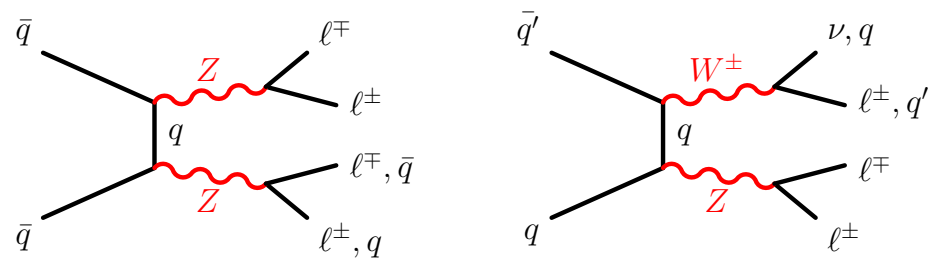

Figure 3.8: Examples of $S M Z V$ production. Left is $Z Z$ production, while right is $Z W$ production. The $Z$ and $W$ bosons that characterize this $S M$ background are highlighted in red.
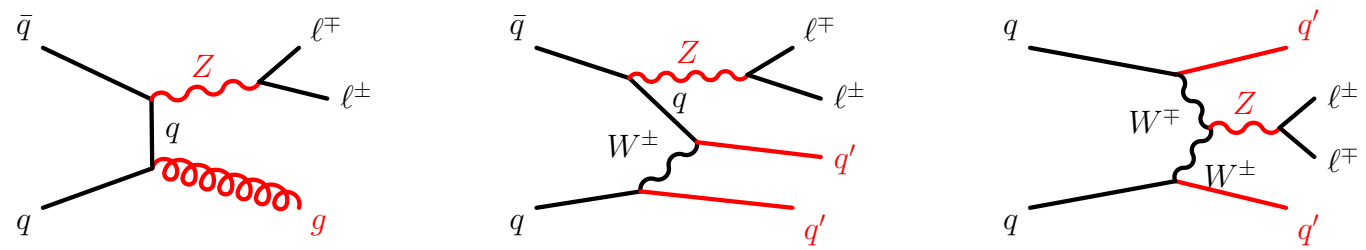

Figure 3.9: Examples of $S M Z+$ jets production. Left diagram shows $Z$ with hard-scattered gluon, middle is the $Z$ bremsstrahlung, and right shows the vectorboson fusion (VBF) process. The particles that characterize this SM background are highlighted in red, where the quarks and gluons produce jet signatures.

boson mass, $m_{Z}$. This fact could be used to identify these $Z V$ events.

\subsubsection{ZX (Z+jets)}

SM $Z X$ events are similar to $Z V$, but with the absence of the extra vector boson, and instead have hadronic activity resulting from either another QCD object as a result of the hard-scatter, or processes such as vector boson fusion (VBF) or $Z$-boson bremsstrahlung. Examples of such cases are depicted in Figure 3.9. 

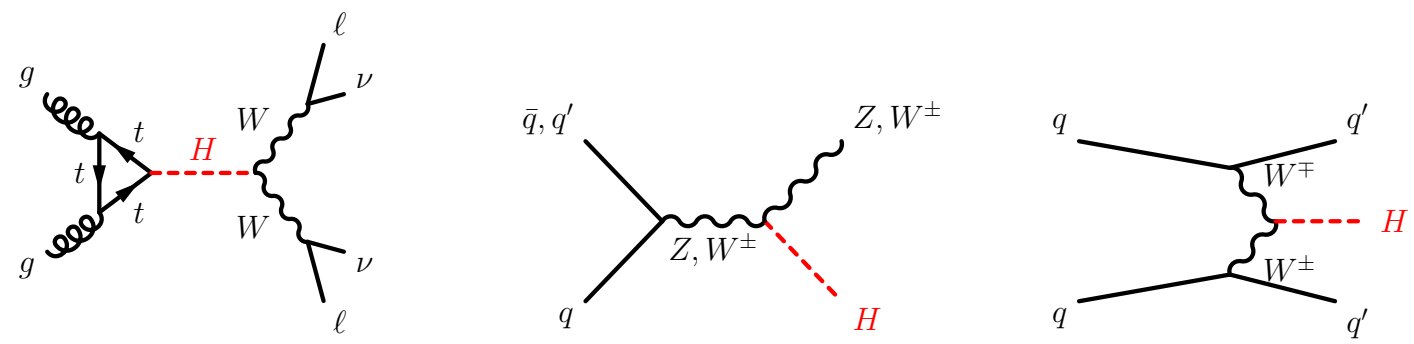

Figure 3.10: Examples of SM Higgs production. Left diagram shows the Higgs production via gluon fusion, then decay via $W W$, middle is the higgsstrahlung, and right is the VBF process. The Higgs boson that characterizes this SM background is highlighted in red.

\subsubsection{Higgs}

Some SM Higgs have decay processes very similar to the SUSY events of interest. This mainly comes from $H \rightarrow W W$ channel from gluon fusion, but could also come from VBF or higgsstrahlung, as depicted in Figure 3.10. In the first case, the final state is very similar to $W W$ background in Section 3.5.2, and thus difficult to separate from the SUSY signal. However, due to the relatively low cross section, these Higgs events are not a significant source of background for the analysis.

\subsubsection{Fake Leptons}

Fake leptons are other SM processes that mimic leptonic behaviour that could be misclassified as leptons by the detector. This could come from misidentified jets, leptons from heavy-flavour decay (non-prompt leptons) or photon-conversions. This could lead to the possibility of misidentifying a seemingly innocuous process without two final state leptons as a signal event. 


\section{METHODOLOGY}

The hunt for EW SUSY signatures with the ATLAS detector at the LHC is a complex, multi-step process. This is due to the fact that the proton collisions at the LHC involve many particle interactions, not all of which are relevant to the SUSY signal of interest. Due to the weakly-interacting nature of the postulated stable SUSY particle, the LSP, the ATLAS detector is not capable of identifying it. As well, it cannot easily distinguish SUSY signal final states from SM processes that mimic these SUSY interactions as outlined in Section 3.5.

The goal of the search is to observe an excess of events above the SM expectation, and it was performed by a small team within the SUSY working group of the ATLAS collaboration. I was involved as one of the members of the team that focused on the EW SUSY signature with two final-state leptons and missing energy. I took on responsibilities of some key components of the analysis which will be presented in later chapters.

The methodology used for the analysis is outlined in this chapter. First, collision data and simulated data used for this analysis are introduced. Then, the definitions 
of objects, and their selection criteria are detailed. The strategy of the analysis is subsequently outlined. Finally, a discussion of systematic uncertainties concludes this methodology chapter.

\subsection{Data and Monte Carlo Samples}

Signatures for SUSY processes could be observable at LHC energies by taking real collision data, and comparing their event properties to the expected signatures from simulated SM and SUSY data. The details of these two types of data are outlined in the following subsections.

\subsubsection{Data Collection}

For this thesis work, $p p$ collision data taken with the ATLAS detector between March 2012 and December 2012 are used. These data correspond to a total integrated luminosity of $20.3 \mathrm{fb}^{-1}$ at the centre-of-mass energy of $\sqrt{s}=8 \mathrm{TeV}$ (see Table 2.1).

The collision data are processed into an end-user analysis format called D3PD as described in Section 2.2.1. The D3PD version used for collision data is p1542. The data D3PD then go through a series of quality checks to ensure they do not contain poor-quality events. These checks are described below.

\section{Good Runs List (GRL)}

A GRL is a list of a subset of the collision data collection (called lumiblocks) when all detector components were operational without any abnormalities. It is produced centrally by the data preparation group within the ATLAS collaboration and is tailored for specific categories of physics analyses since some analysis may use different 
detector components. Any lumiblocks that are not included in the GRL will likely have unreliable detector information, and therefore may bias the analysis. This requirement rejects approximately $4 \%$ of the total data.

\section{Detector-based quality criteria}

These criteria are checked after the GRL, and further reject events that may lead to unreliable event information due to detector effects. They include: Tile calorimeter cleaning, which rejects events with jets pointing to a space where misbehaving read-out channels were improperly masked; checks for possible incomplete events corresponding to a lumiblock right after a trigger-timing control (TTC) was reset in middle of a data run; and checks for noise bursts within LAr calorimeter. Together with the GRL check, approximately $5 \%$ of the total data are rejected after this stage.

\section{Event-based quality criteria}

After the detector-based quality checks, each event is further scrutinized for its quality: checks for events with jets that are labeled as VeryLooseBad by the jet identification algorithm, or jets pointing to a problematic calorimeter segment; checks to ensure that the event has a primary vertex with at least five tracks associated with it; and checks for muons that are potentially mis-measured or those identified as muons from cosmic rays. Together with the GRL and the detector-based quality checks, approximately $7 \%$ of the total data are rejected after this stage.

\subsubsection{Triggers}

This thesis focuses only on the final states with two leptons. Only a subset of all $p p$ collisions are triggered as events of interest in order to reduce the number of input 


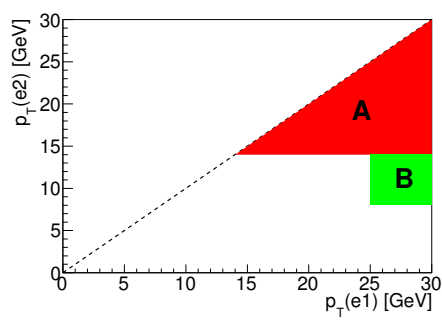

(a) ee channel

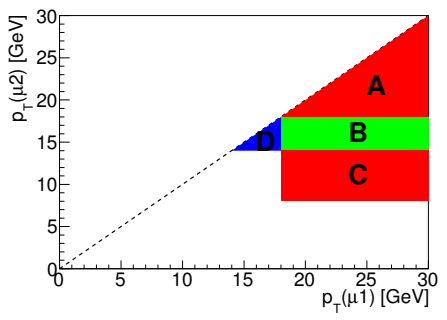

(b) $\mu \mu$ channel

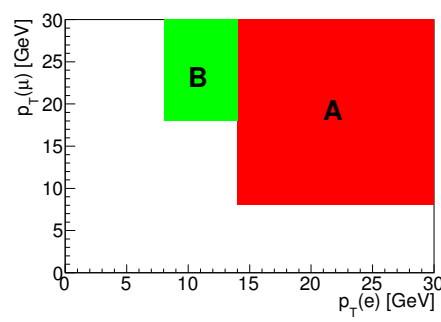

(c) $e \mu$ channel

Figure 4.1: A summary of trigger regions in the dilepton transverse momentum $\left(p_{\mathrm{T}}\right) 2 D$ parameter space for ee (a), $\mu \mu(b)$ and e $\mu$ (c) channels. For (a) and (b), the $x$-axis (y-axis) is the $p_{\mathrm{T}}$ of the leading (subleading) lepton, therefore the trigger regions are only defined within the lower half of the diagonal dashed line.

events (to $400 \mathrm{~Hz}$ as described in Section 2.2.1) since many $p p$ collisions include other events that do not involve leptons.

The trigger system selects events based on a transverse momentum $p_{T}$ (or energy $\left.E_{T}\right)$ of an object. Event Filter (EF) triggers are used, where an offline reconstruction algorithm identifies an object, and matches it with an object identified by the level1 (L1) trigger. In this analysis, dilepton triggers are used, where two leptons are simultaneously triggered. Since the final-state leptons are either electrons or muons, there are three different combinations: electron-electron $(e e)$, electron-muon $(e \mu)$ or muon-muon $(\mu \mu)$. Depending on these combinations, and depending on the $p_{T}$ of the leptons, different triggers are used. The lowest possible $p_{T}$ threshold of the triggers were selected in order to collect the maximal amount of input events. An additional "offline" $p_{T}$ threshold is applied a few GeV above the trigger $p_{T}$ to ensure good trigger efficiency. Figure 4.1 and Table 4.1 summarizes the triggers used. 


\begin{tabular}{|c|l|l|l|l|}
\hline \multicolumn{2}{|c|}{} & $\begin{array}{l}p_{T}(\ell 1) \\
{[\mathrm{GeV}]}\end{array}$ & $\begin{array}{l}p_{T}(\ell 2) \\
{[\mathrm{GeV}]}\end{array}$ \\
\hline \multirow{2}{*}{$e e$ channel } & Region A & EF_2e12Tvh_loose1 & 14 & 14 \\
& Region B & EF_e24vh_medium1_e7_medium1 & 25 & 8 \\
\hline$\mu \mu$ channel & Region A & EF_mu18_tight_mu8_EEFS & 18 & 8 \\
& Region B & EF_mu18_tight_mu8_EEFS or EF_2mu13 & $18(14)$ & $8(14)$ \\
& Region C & EF_mu18_tight_mu8_EEFS & 18 & 8 \\
& Region D & EF_2mu13 & 14 & 14 \\
\hline \multirow{2}{*}{$e \mu$ channel } & Region A & EF_e12Tvh_medium1_mu8 & 14 & 8 \\
& Region B & EF_mu18_tight_e7_medium1 & 18 & 8 \\
\hline
\end{tabular}

Table 4.1: Summary of triggers used for the analysis. The trigger follows the following nomenclature (separated by an underscore "—"): The first "EF" denotes the trigger used is EF trigger; triggering lepton (e or $\mu$ ) followed by an online $p_{\mathrm{T}}$ threshold. Additional flags may be appended ("T" indicates the small difference in L1 and EF threshold, " $v$ " indicates $\eta$-dependent threshold at L1, and " $h$ " indicates an upper threshold of energy in hadronic calorimeter at L1); and lepton identification criteria. $p_{\mathrm{T}}$ values in the last two columns (where $\ell 1$ and $\ell 2$ are leading and subleading leptons, respectively) represent transverse momentum threshold values for offline objects.

\subsubsection{Monte Carlo Samples}

Monte Carlo (MC) samples are used to estimate the expected number of events for a particular type of initiating particle or its interaction. These simulated samples are important since collision data do not easily distinguish one type of interaction from another. MC simulation in general consists of three steps: event generation that simulates the initial production of particles of interest through $p p$ collisions; hadronization that simulates the particle interaction before entering the detector material; and detector simulation that simulates the particle interaction with the detector material and decay process. Depending on the underlying process, different types of event generation and hadronization models are used (see Table 4.2 and Table 4.3). The detector simulation is done either by the full detector simulation using GEANT4 [47], or through a fast, parameterized simulation called ATLFAST- 
II [48].

As with the collision data samples, the MC samples are processed into a D3PD format using version p1512. Event-based quality criteria as described in Section 4.1.1 are applied and triggered as described in Section 4.1.2. In addition, a pile-up distribution reweighting and a MC-specific event weight are necessary to properly simulate the $p p$ collisions at the LHC.

\section{SM Background MC Samples}

The SM background categories were introduced in Section 3.5. For each category, dedicated MC samples are used for specific processes, and they are summarized in Table 4.2.

\section{Signal MC Samples}

As described in Section 3.4, there are two types of signal models considered for this analysis. Simplified Models are diagram-based, therefore for each diagram in Figure 3.5, a dedicated MC sample is generated. For pMSSM models, separate MC samples are used for direct gaugino production with decays involving sleptons and without sleptons. As these models have free parameters as explained in Section 3.4, numerous samples are produced for each model, each with different combinations of masses to form a "grid" in this mass parameter space. This enables the scanning of the model through the grid. The signal MC samples are generated with HERWIG $++[59]$ and the hadronization is modeled with UEEE3 [60]. Detector simulation is done with ATLFAST-II. Table 4.3 summarizes the signal MC samples. 


\begin{tabular}{|c|c|c|c|}
\hline Category & Process & MCID & Generator / Hadronization \\
\hline \multirow[t]{2}{*}{ Top } & $t \bar{t}$ & 105200 & MC@NLO [49] / Jimmy [50] \\
\hline & $W t$ & 108346 & MC@NLO / Jimmy \\
\hline \multirow[t]{4}{*}{$W W$} & $W W$ & $126928-36$ & POWHEG [51] / Pythia8 [52] \\
\hline & $g g \rightarrow W W$ & $169471-9$ & gg2WW [53] / Jimmy \\
\hline & $W W+j j$ & $126988-9$ & SHERPA [54] \\
\hline & $V V \rightarrow l \nu q q$ & $157817-9$ & SHERPA \\
\hline \multirow[t]{6}{*}{$Z V$} & $W Z$ & $129477-94$ & POWHEG / Pythia8 \\
\hline & $Z Z \rightarrow 4 \ell$ & $126937-42$ & POWHEG / Pythia8 \\
\hline & $Z Z \rightarrow 2 \ell+2 \nu$ & $126949-51$ & POWHEG / Pythia8 \\
\hline & $g g \rightarrow Z Z$ & $116600-3$ & gg2ZZ [55] / Jimmy \\
\hline & $V V \rightarrow l l q q$ & $157814-6$ & SHERPA \\
\hline & $Z W W, Z Z Z$ & $167007-8$ & MADGRAPH [56] / Pythia6 [57] \\
\hline \multirow[t]{7}{*}{$Z X$} & $m_{l l}<40 \mathrm{GeV}(\mathrm{DY})$ & $173041-6$ & SHERPA \\
\hline & $m_{l l}<60 \mathrm{GeV}(\mathrm{DY})$ & $147770-2$ & SHERPA \\
\hline & $m_{l l}>60 \mathrm{GeV}(Z e e)$ & $117650-55$ & ALPGEN [58] / Pythia6 \\
\hline & $m_{l l}>60 \mathrm{GeV}(Z \mu \mu)$ & $117660-65$ & ALPGEN / Pythia6 \\
\hline & $m_{l l}>60 \mathrm{GeV}(Z \tau \tau)$ & $117670-75$ & ALPGEN / Pythia6 \\
\hline & $m_{l l}>60 \mathrm{GeV}(Z c c)$ & 110805-16 & ALPGEN / Pythia6 \\
\hline & $m_{l l}>60 \mathrm{GeV}(Z b b)$ & $110817-28$ & ALPGEN / Pythia6 \\
\hline \multirow[t]{4}{*}{ Higgs } & $W H$ & $\begin{array}{c}160255,160755 \\
161105\end{array}$ & Pythia8 \\
\hline & $Z H$ & $\begin{array}{c}160305,160805 \\
161155\end{array}$ & Pythia8 \\
\hline & $\mathrm{VBF}$ & $\begin{array}{c}160205,160705 \\
161055\end{array}$ & POWHEG / Pythia8 \\
\hline & $g g \rightarrow H$ & $\begin{array}{c}160155,160655 \\
161005\end{array}$ & POWHEG / Pythia8 \\
\hline
\end{tabular}

Table 4.2: List of SM background MC samples generated at $\sqrt{s}=8 \mathrm{TeV}$. The first column is the general SM process category, with the second column indicating the specific process within it. The third column lists the ID number for the MC samples corresponding to the process. The fourth column lists the model used for the event generation and hadronization.

\subsection{Object Definitions and Event Selection}

The ATLAS detector employs sophisticated algorithms to identify and distinguish different types of particle interactions using various components of the detector. The 


\begin{tabular}{c|r|r}
\hline Category & Process & \multicolumn{1}{c}{ MCID } \\
\hline Simplified & $\tilde{\chi}_{1}^{ \pm} \tilde{\chi}_{1}^{ \pm}$via sleptons (Figure 3.5a) & $144902-27,157969-86,176558-73$ \\
Model & $\tilde{\ell} \tilde{\ell}_{\text {production (Figure 3.5b) }}$ & $166501-658,175420-583,177423-496$ \\
& $\tilde{\chi}_{1}^{ \pm} \tilde{\chi}_{1}^{ \pm}$via W (Figure 3.5c) & $176322-479,164374-423$ \\
& $\tilde{\chi}_{1}^{ \pm} \tilde{\chi}_{2}^{0}$ via WZ (Figure 3.5d) & $164324-164373$ \\
\hline pMSSM & with sleptons & $164949-165740$ \\
& without sleptons & $186000-186099$ \\
\hline
\end{tabular}

Table 4.3: List of SUSY signal MC samples generated at $\sqrt{s}=8 \mathrm{TeV}$. The first column is the general signal SUSY model category, with the second column indicating the specific process within it. The third column lists the ID number for the MC samples corresponding to the process.

detector then reconstructs an "object" from a collection of these detector signals. The definitions of objects that are relevant to this analysis, their properties, and how they are identified by the ATLAS detector are described further in detail in this section.

These definitions are implemented in an offline analysis software package and selected on an event-by-event basis. The software package, called SUSYTools, is commonly used among all SUSY searches within the ATLAS collaboration. The version used for this thesis work is SUSYTools-00-03-04.

\subsubsection{Leptons}

In this analysis, leptons refer to either electrons or muons, although tau leptons are also identified to be vetoed in order to be consistent with the trilepton analysis [61] and ditau analysis [45]. These leptons are visible and can be identified using the ATLAS detector. The identification of leptons consists of two steps: first, baseline selection criteria are applied to choose candidate leptons; then a more stringent selection is made to select so-called signal leptons. Table 4.4 summarizes the definitions for leptons. 


\begin{tabular}{l|c|c|c}
\hline & Electron & Muon & Tau \\
\hline Identification & medium++ & STACO loose & Loose \\
$p_{\mathrm{T}}$ & $>10 \mathrm{GeV}$ & $>10 \mathrm{GeV}$ & $>20 \mathrm{GeV}$ \\
$|\eta|$ & $<2.47$ & $<2.40$ & $<2.47$ \\
\hline & \multicolumn{3}{|c}{ Signal } \\
\hline Identification & tight++ & STACO loose & Medium \\
$\left|d_{0}^{P V}\right|$ & $<5 \sigma_{d_{0}^{P V}}$ & $<3 \sigma_{d_{0}^{P V}}$ & - \\
$\left|z_{0}^{P V} \times \sin \theta\right|$ & $<0.4 \mathrm{~mm}$ & $<1 \mathrm{~mm}$ & - \\
$p_{\mathrm{T}}^{\text {cone } 30} / p_{\mathrm{T}}$ & $<16 \%$ & $<12 \%$ & - \\
$E_{T}^{\text {cone30 }} / p_{\mathrm{T}}$ & $<18 \%$ & - & - \\
\hline \hline
\end{tabular}

Table 4.4: Definitions of lepton objects.

\section{Electrons}

Electrons are identified using the ID and the calorimeter system. They are characterized by shower shapes in the EM calorimeter, energy leakage into the hadronic calorimeter, and tracking information in the ID [62]. Since electrons are charged particles, they leave a trail of hits (a "track") in the tracking detector. By the direction with which they bend, the charge can be identified (anti-electrons are normally called positrons).

A baseline electron requires that it is identified using medium++ criteria $^{1}$ using the official identification tool provided by the egamma performance group. The identification efficiency ${ }^{2}$ of medium++ is $80 \%$ to $95 \%$ depending on the $E_{\mathrm{T}}$ of the elctrons [63]. It must also require its transverse momentum $p_{\mathrm{T}}$ to be above $10 \mathrm{GeV}$, and within the $\eta$ region dedicated to precision measurements, $|\eta|<2.47$.

A signal electron requires the identification using tight++ criteria (where the $E_{\mathrm{T}}$-dependent identification efficiency is $65 \%$ to $90 \%$ [63]), and also on the impact

\footnotetext{
${ }^{1}$ the object identification criteria in ATLAS are labelled as loose, medium, tight, in increasing order of background rejection at expense of efficiency loss.

${ }^{2}$ background rejection factors are process dependent, therefore are not quoted.
} 


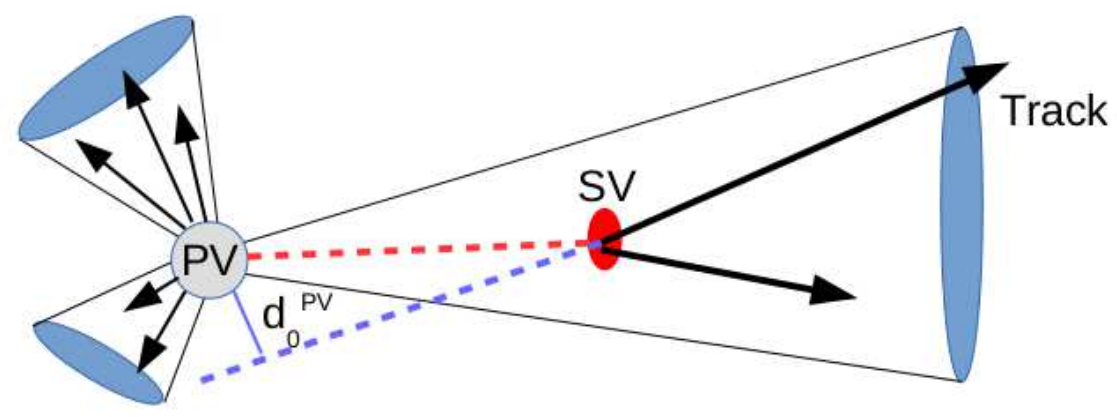

Figure 4.2: Illustration of impact parameter in the transverse plane. A particle originating from a secondary vertex (SV) will have a track displaced from a primary vertex $(P V)$ where the colliding beams cross perpendicular to the page, and the distance of closest approach in the transverse plane (denoted $d_{0}^{P V}$ ) is the impact parameter.

parameter designed to test the origin of the identified particle (see Figure 4.2 for an illustration) as well as isolation criteria. The impact parameters $d_{0}^{P V}$ and $z_{0}^{P V}$ describe the point of closest approach of the reconstructed track to the Primary Vertex (PV) in the transverse plane and along the beam-line, respectively. If the track belongs to particles created from the secondary vertex (SV), then the impact parameter is expected to be large (on the order of $\mathrm{mm}$ ). The requirements on the impact parameters are $\left|d_{0}^{P V}\right|<5 \sigma_{d_{0}^{P V}}$ (within 5 standard deviations of the impact parameter $\left.d_{0}^{P V}\right)$ and $\left|z_{0}^{P V} \times \sin \theta\right|<0.4 \mathrm{~mm}$, respectively. Isolation criteria include: a cut on $p_{\mathrm{T}}^{\text {cone30}}$, a scalar sum of transverse momentum $p_{\mathrm{T}}$ above $400 \mathrm{MeV}$ within a cone around the candidate with a size of $\Delta R<0.3$ (where $\Delta R=\sqrt{\Delta \phi^{2}+\Delta \eta^{2}}$ ), to be less than $16 \%$ of the $p_{\mathrm{T}}$ of the identified electron; and a cut on $E_{\mathrm{T}}^{\text {cone30 }}$, a sum of transverse energy deposit $E_{\mathrm{T}}$ in the surrounding calorimeter cluster within a cone around the candidate with a size of $\Delta R<0.3$, to be less than $18 \%$ of the $p_{\mathrm{T}}$ of the identified electron. 


\section{Muons}

Muons are identified using the ID and the muon spectrometer. As with electrons, the tracking is done with the ID, but also combined with the spectrometer using the STACO algorithm $[62,64]$. Due to the larger mass of the muons compared to the electrons, they do not deposit significant energy in the calorimeter system. Instead, their momentum is measured with the spectrometer.

For the baseline selection, STACO loose identification criteria are required as provided by the muon combined performance (MCP) group, where the identification efficiency is $\sim 99 \%$ [65]. The transverse momentum must be greater than $10 \mathrm{GeV}$, and within pseudorapidity region $|\eta|<2.40$.

A signal muon must also pass impact parameter requirements similar to electrons, with $\left|d_{0}^{P V}\right|<3 \sigma_{d_{0}^{P V}}$ and $\left|z_{0}^{P V} \times \sin \theta\right|<1 \mathrm{~mm}$. The isolation quantity similar to the electrons, $p_{T}^{\text {cone } 30}$, is required to be less than $12 \%$ of the $p_{T}$ of the identified muon.

\section{Taus}

Tau leptons are identified using a multivariate technique called Boosted Decision Tree (BDT) to distinguish between a tau and a jet (jetBDT) and between a tau and an electron (electronBDT) [66].

A baseline tau requires that the selection criteria from BDT to be loose for both jet and electron. It also requires $p_{T}$ greater than $20 \mathrm{GeV},|\eta|$ range within 2.47 , and the number of associated tracks either 1 or 3 (so-called one-pronged or three-pronged decays). For signal taus, medium selection is required for jetBDT. The identification efficiencies for loose and medium selections are $\sim 70 \%$ and $\sim 60 \%$ respectively [66]. 


\begin{tabular}{|c|c|c|c|}
\hline & \multicolumn{3}{|c|}{ Jets } \\
\hline & \multicolumn{3}{|c|}{ Baseline } \\
\hline Identification & \multirow{3}{*}{\multicolumn{3}{|c|}{ 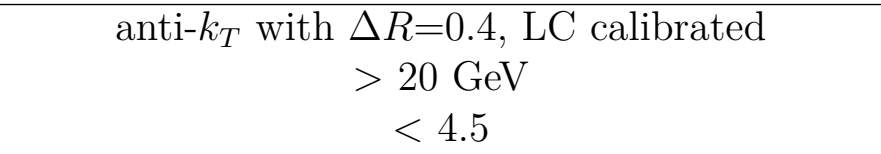 }} \\
\hline$p_{\mathrm{T}}$ & & & \\
\hline$|\eta|$ & & & \\
\hline & Light Jets & b-Jets & Forward Jets \\
\hline$p_{\mathrm{T}}$ & $>20 \mathrm{GeV}$ & $>20 \mathrm{GeV}$ & $>30 \mathrm{GeV}$ \\
\hline$|\eta|$ & $<2.4$ & $<2.4$ & $2.4<|\eta|<4.5$ \\
\hline$b-\operatorname{tag}(\mathrm{MV} 1)$ & $\leq 0.3511$ & $>0.3511$ & - \\
\hline JVF & $>0$ if $p_{\mathrm{T}}<50 \mathrm{GeV}$ & - & - \\
\hline
\end{tabular}

Table 4.5: Definitions of jet objects.

\subsubsection{Jets}

Jets refer to hadronic activity within the detector. These are identified using the ID and the calorimeter system, using the anti- $k_{T}$ algorithm with distance parameter of $\Delta R=0.4$ [67]. As with the leptons, baseline criteria are enforced before selecting signal jets. Baseline jets are the subset of identified jets with the transverse momentum greater than $20 \mathrm{GeV}$ where the local-cluster (LC) weighting calibration is employed [68], and fall within pseudorapidity region $|\eta|<4.5$. The identification efficiency of jets is $80 \%$ for $p_{\mathrm{T}} \sim 20 \mathrm{GeV}$ and $99 \%$ for $p_{\mathrm{T}}>30 \mathrm{GeV}[68]$.

For the signal jets, three sub-categories are identified depending on the behaviour and flavour of the jets. They are summarized in Table 4.5.

\section{Light Jets}

These refer to jets that are likely initiated by light flavoured quarks or gluons (i.e. not b-quarks - see the b-jets description below for the discriminating variable). Due to the limitation of b-jet identification, light jets are identified only within the central detector component $(|\eta|<2.4)$. In addition, the requirement of jet vertex fraction 


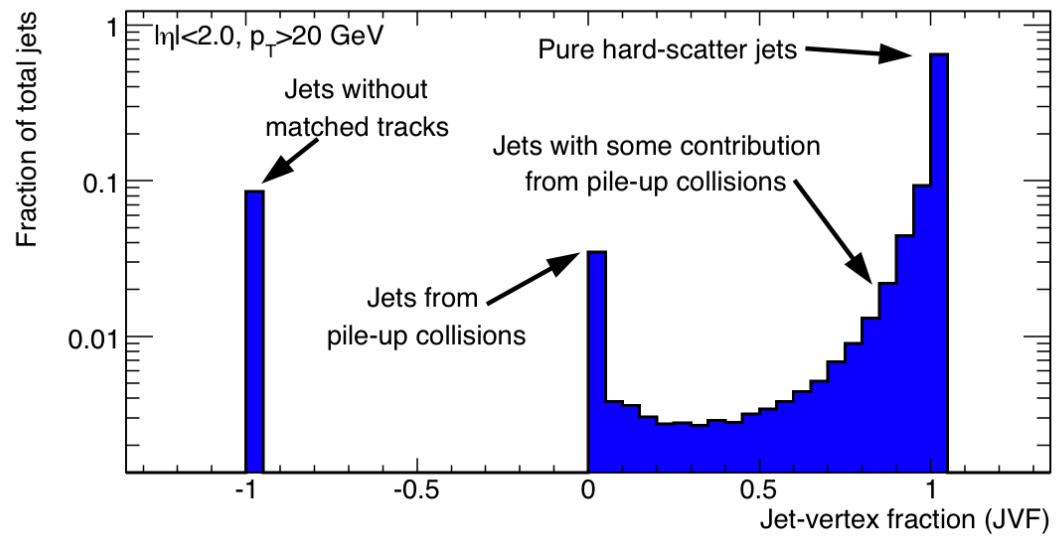

Figure 4.3: Distribution of JVF.

(JVF) is enforced. JVF is a quantity that is designed to distinguish between jets from hard-scattered events and pile-up jets, and it is defined as a fraction of track momenta from the PV compared to all of the momenta associated to the jet. An example JVF distribution is illustrated in Figure 4.3. The JVF requirement is found to be effective only if the jets are relatively low-energy $\left(p_{\mathrm{T}}<50 \mathrm{GeV}\right)$.

\section{b-Jets}

These refer to jets that are likely initiated by b-quarks. Since a B-hadron has a long decay time, it creates a displaced vertex before it decays. A neural-network of several b-tagging techniques is used to identify these processes, called MV1 [69]. This MV1 technique requires the jets to be within the range $|\eta|<2.4$ because it relies on tracking information. Jets within this $\eta$ range, and with MV1 score of greater than 0.35, corresponding to identification efficiency of $80 \%$, are categorized as b-jets, while light jets are categorized by scores of $\leq 0.35$. At this working point, the mis-tag rate (the probability that a light jet is mistakingly tagged as a b-jet) is between $1 \%$ and $5 \%$ depending on the jet $p_{\mathrm{T}}[70]$. 


\section{Forward Jets}

These refer to jets that are created in the forward region of the detector $(2.4<|\eta|<$ 4.5). Since tracking is not possible in this region, neither b-jet identification nor JVF requirement is enforced. Instead, the threshold $p_{\mathrm{T}}$ is increased to $30 \mathrm{GeV}$ to ensure the jet comes from a hard-scattered event.

\subsubsection{Overlap Removal of Leptons and Jets}

The baseline selection of leptons and jets provides a reasonable description of real objects associated to a physical process of interest. However, these objects could still be mislabeled or ambiguous if they are close to other identified objects. These overlapped objects could bias the signal selection. For this reason, these overlapped objects are removed before applying signal object selection criteria as this will ensure that these objects are well isolated. They are done in sequential order as follows (where $\Delta R=\sqrt{(\Delta \phi)^{2}+(\Delta \eta)^{2}}$ ):

1. If $\Delta R$ between two electrons is within 0.05 , remove the electron with lower transverse momentum,

2. If $\Delta R$ between a jet and an electron is within 0.2 , remove the jet,

3. If $\Delta R$ between a tau and either electron or muon is within 0.2 , remove the tau,

4. If $\Delta R$ between a jet and electron or muon is within 0.4 , remove the electron or the muon,

5. If $\Delta R$ between an electron and muon is within 0.01 , remove both the electron and the muon,

6. If $\Delta R$ between two muons is within 0.05 , remove both muons,

7. If $\Delta R$ between a jet and signal tau is within 0.2 , remove the jet. 
The above $\Delta R$ values are motivated by the respective identification algorithms. For example, the jet identification uses $\Delta R=0.4$ (see Table 4.5), and the different detector components used for lepton identifications lead to the differences in the $\Delta R$ values for the lepton overlaps.

In order to suppress low-mass backgrounds such as the Drell-Yan process and resonant production of $\Upsilon$ and $J / \psi$, a remaining baseline of oppositely-charged ee or $\mu \mu$ pairs with invariant mass $m_{\ell \ell}$ less than $12 \mathrm{GeV}$ is removed before application of signal lepton selection.

\subsubsection{Missing Energy}

Even within the realm of SM particles, there are weakly-interacting particles that escape the detector without being detected (namely the neutrinos). As mentioned in Chapter 3, the LSP is also weakly-interacting, and therefore escapes the detector. They are however essential component of a final-state, and therefore need to be identified.

Since there is no direct way to measure these particles, they are deduced by finding the imbalance in the sum of momenta in transverse plane, as depicted in Figure 4.4. The refined calibration method, or RefFinal, is used for missing energy computation. It consists of several components: leptons $(e, \mu, \tau)$ and photons with $p_{T}>10 \mathrm{GeV}$, jets with $p_{T}>20 \mathrm{GeV}$, and other clusters of energy within $|\eta|<4.9$, called the "soft-term" [71]. This imbalance is calculated in the $\mathrm{x}$ - and the $\mathrm{y}$-components independently, then combined together to form so-called missing transverse momentum, or equivalently called missing transverse energy when omitting directionality. It is 

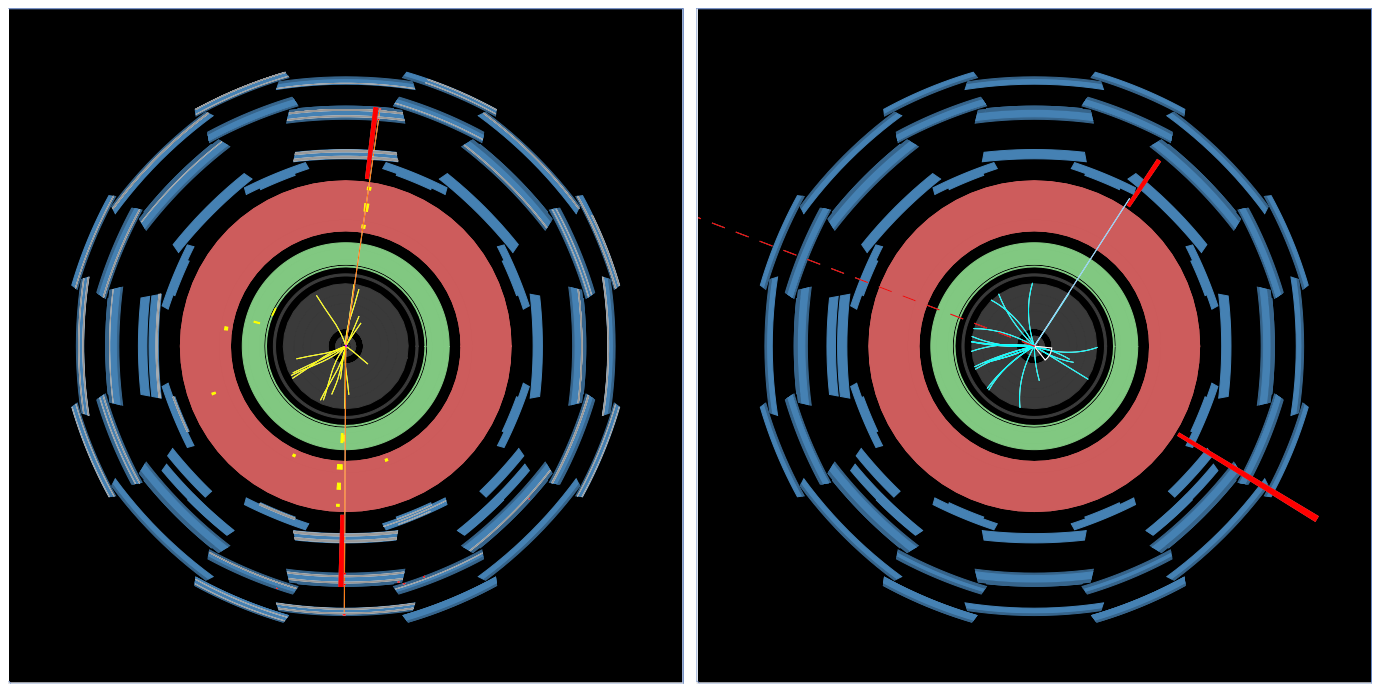

Figure 4.4: An event with balanced energy deposit in transverse plane of the detector (left). Event with unbalanced energy deposit (right). A red dash indicates the direction of the missing energy.

denoted as $E_{T}^{\text {miss }}$, as follows:

$$
\begin{gathered}
E_{x,(y)}^{\text {miss }}=E_{x,(y)}^{\text {miss,e }}+E_{x,(y)}^{\text {miss }, \mu}+E_{x,(y)}^{\text {miss }, \tau}+E_{x,(y)}^{\text {miss }, \gamma}+E_{x,(y)}^{\text {miss }, j e t s}+E_{x,(y)}^{\text {miss }, \text { soft }}, \\
E_{T}^{\text {miss }}=\sqrt{\left(E_{x}^{\text {miss }}\right)^{2}+\left(E_{y}^{\text {miss }}\right)^{2}} .
\end{gathered}
$$

This missing transverse energy is a sum of all invisible particles. For example, if a final-state consisted of both $\tilde{\chi}_{1}^{0}$ and $\nu$, then it will correspond to a sum of the two $p_{\mathrm{T}}$ of the invisible particles, and it is not possible to separate the individual contributions from the two invisible particles. In addition, the imbalance of energy could include energy loss due to the resolution of the detector, possibly skewing the measurement. 


\subsection{Analysis Strategy}

In this section, the major steps of the search strategies for EW SUSY signatures are outlined, where the responsibilities for each of these steps were divided among group members. Of the steps listed below, I have made personal contributions to signal region definition (Section 4.3.2) for $\mathrm{SR}-W W$, and statistical interpretation (Section 4.3.5) for the pMSSM models.

\subsubsection{Blinding of the Data}

A signature for a SUSY interaction can be found in the ATLAS detector by looking for an excess of events in collision data compared to the expected SM yield from the $\mathrm{MC}$ simulation in a region called a signal region ( $\mathrm{SR}$ - see next subsection). This $\mathrm{SR}$ definition can be biased if it is designed with a priori knowledge of the behaviour of the collision data in these regions. In order to avoid this potential bias, the collision data are ignored (or blinded), and the SR regions are designed by analyzing the simulated data only.

\subsubsection{Signal Region Definition}

The signal region (SR) is a region where the expected SUSY events are most likely to be enhanced and visible. That is, the SUSY signal is maximized while the SM background is minimized. Since some sparticle interactions are very similar to SM background events, the series of kinematic selections are not trivial and must be

optimized in order to separate the two types of processes. Depending on the types of SUSY interactions, three separate SR are independently designed $\left(\mathrm{SR}-m_{\mathrm{T} 2}, \mathrm{SR}-W W\right.$ 
and SR-Zjets). I have made personal contributions in designing of SR- $W W$ regions.

Details of the SR optimization are discussed further in Chapter 5.

\subsubsection{Estimation of the Backgrounds}

As mentioned earlier, SM background events can mimic SUSY signatures, and therefore understanding these backgrounds is a very important part of the analysis. Many types of interactions take place in real collision data, but there is no a priori knowledge of which processes will occur in any given event. By using theoretical models and simulated data, the compositions of different interactions can be disentangled. This is done by defining what is called a control region (CR) for each of the dominant backgrounds (as outlined in Section 3.5) found in a SR. As depicted in Figure 4.5, a CR is complimentary to a SR so that blinded data are excluded (remember that real collision data in the SR are blinded). By comparing the collision data and simulated data in a $\mathrm{CR}$, the behaviour and agreement of the modelling of these backgrounds can be checked. Then the yield in the $\mathrm{CR}$ is interpolated to the SR to obtain the expected yield for each background process in the SR.

\subsubsection{Unblinding of the Data}

Once the SR definitions are completed and SM background composition for these regions are determined, the collision data can then be unblinded and analyzed in the SR. At this point, various particle behaviours in the SR are checked, by plotting particle kinematics and comparing collision data with simulated data. Chapter 6 shows some of these kinematic distributions demonstrating the agreement between the data and the MC. 


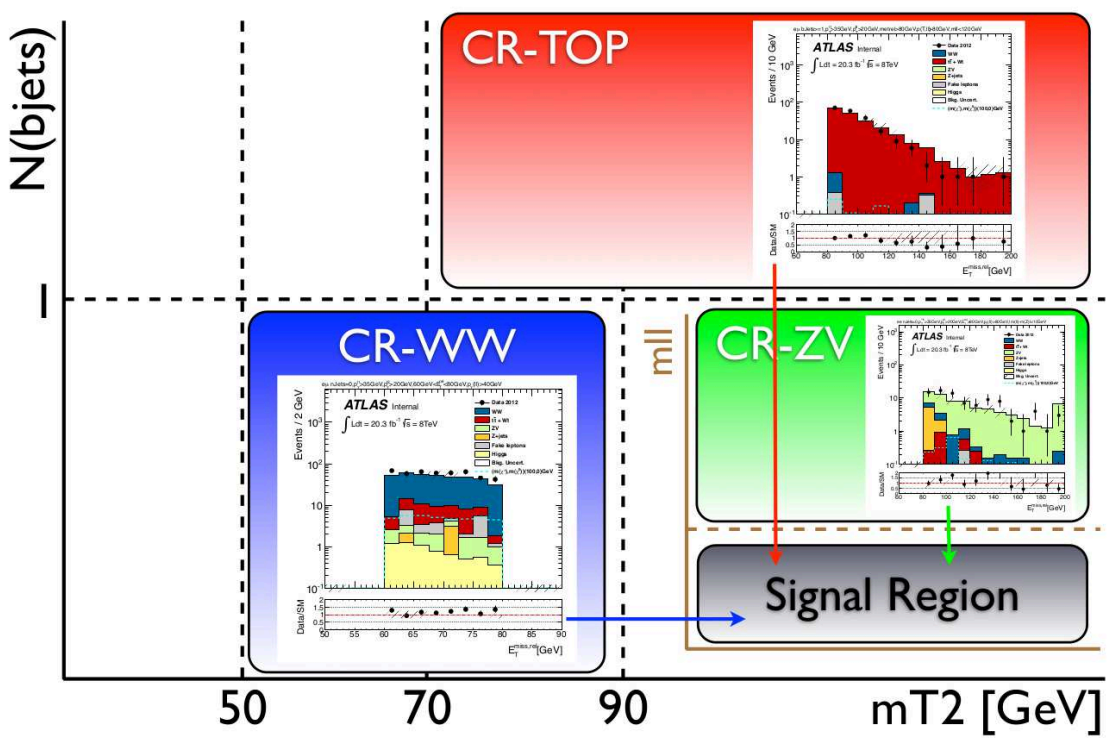

Figure 4.5: Illustration of control region (CR) definition. A CR is defined such that it is close to the $S R$, but without an overlap into the blinded region. $C R$ enhances a particular background yield. For example, decreasing the $m_{\mathrm{T} 2}$ requirement, $W W$ contribution is enhanced (labelled $C R$-WW), while increasing the number of b-jets enhances the top contribution (labelled CR-TOP). Finally, higher $m_{\ell \ell}$ requirement enhances $Z V$ contribution (labelled $C R-Z V$ ).

\subsubsection{Statistical Interpretation}

Once the data are unblinded in the SR, statistical methods can be employed to interpret the results to either validate or refute a SUSY model. This is achieved by means of hypothesis testing, and it is divided into three steps: backgroundonly testing to check the background composition in the SR as determined before unblinding of the data, a discovery test that will try to reject a hypothesis that no new physics beyond the SM exists, and exclusion test that will set upper limits to the parameters of a particular SUSY model (such as sparticle mass) should no excess be observed. See Chapter 6 for further details. 


\subsection{Systematic Uncertainties}

Systematic uncertainties on the measurement of particle properties affect the estimation of background and signal events in the SR. For some cases, there are also scale factors $(\mathrm{SF})$ applied to the MC to correct for the differences in the simulation to the data, or rescaling factors that account for the calibration of physical objects in data. These factors are also accompanied by their own systematic uncertainties and must be taken into account. These uncertainties also affect the statistical interpretation by altering the likelihood function (see Section 6.1.2) and therefore are important to be understood. These are independent of statistical uncertainties in the SR and arise from several sources, as outlined below. See Section 6.3.3 for the summary of values for the systematic uncertainties.

\subsubsection{Jet Uncertainties}

Energies of jets are calibrated using the ATLAS detector. The energy calibration (Jet Energy Scale or JES) is used to rescale the jet energies in the data, and its uncertainties, provided by the Jet/Etmiss performance group within the ATLAS collaboration, are applied to the $\mathrm{MC}$ and dependent on the momentum and direction of the jets [68]. The calibration also determines the energy resolution of the jets to be applied only to the MC (Jet Energy Resolution or JER). The uncertainties associated to it are applied by smearing the nominal jet energy according to a Gaussian distribution with a width given by momentum-dependent resolution function [72]. 


\subsubsection{Lepton Uncertainties}

Electron energy scale and resolution (EES and EER respectively) are analogous to the jet calibration (JES and JER), where the EES is used to rescale the electron energies in the data and EER is applied only to the MC. These factors and the uncertainties associated to them are provided by the egamma performance group within the ATLAS collaboration [73, 74]. Muon momentum calibration is derived from the muon spectrometer (MS) and ID components of momentum measurements (MMS and MID respectively), and they are used to rescale the muon momentum in the data. These factors and the uncertainties associated to them are provided by the muon combined performance (MCP) group within the ATLAS collaboration [75].

In addition, electron and muon identification efficiencies and trigger efficiencies are included as SF. These scale factors correct for the differences in efficiencies observed between the data and MC simulation and uncertainties on these SF are evaluated. The electron and muon identification SF and their uncertainties are provided by the respective performance groups, while the trigger efficiency uncertainty is obtained by varying the trigger requirements such as trigger matching radius and dependence on number of primary vertices [76].

Uncertainties due to tau reconstruction are found to be negligible by the other researchers contributing to the same analysis.

\subsubsection{Missing Energy Uncertainties}

Since the missing energy is derived from the imbalance of energies measured from leptons and jets, the uncertainties on the individual components are propagated as 
the uncertainty for the missing energy. In addition, the uncertainty on the energy scale of "soft jets" with transverse momentum $7 \mathrm{GeV}<p_{T}<20 \mathrm{GeV}$ is evaluated [71].

\subsection{4 b-tagging Uncertainties}

The identification of b-quark initiated jets uses complex multivariate techniques (as introduced in Section 4.2.2), therefore the uncertainty associated to b-tagging is provided by the b-tagging performance group within the ATLAS collaboration. The uncertainties include b-jet identification efficiency and rejection efficiency of the lightflavoured jets [69].

\subsubsection{Fake Lepton Uncertainties}

The description of the fake leptons was given in Section 3.5.6. The uncertainties come from data-driven methods used for estimating the contribution of these fake leptons as described in Reference [76]. These include an $\eta$ dependence on these fake leptons and statistical error.

\subsubsection{Modelling Uncertainty}

Modelling uncertainties come from the methods used to model particle interactions and theoretical predictions of particle properties. These include: generator uncertainties for MC samples, which are obtained by comparing with alternate generators (for example for top and WW samples, POWHEG and MC@NLO samples are compared); cross section uncertainties calculated for both background and signal processes; and uncertainties on parton distribution function (PDF) of the initial proton beams by using different PDF models. 


\subsubsection{Luminosity Uncertainty}

The uncertainty on the integrated luminosity depends on factors such as beam posi-

tion and behaviour, beam-beam interaction modelling, and pile-up conditions. It is calculated by the ATLAS luminosity group [77], and it is estimated to be $\pm 2.8 \%$. 


\section{Signal Region Optimization}

As described in Section 4.3.2, the optimization of signal regions (SR) is an important stage of the overall search strategy for SUSY signatures. In this analysis, three types of SR are designed to target the specific Simplified Model topologies that were introduced in Section 3.4.2: SR- $m_{\mathrm{T} 2}$ targets chargino pair production with subsequent decays involving intermediate sleptons and slepton pair production (Figure 3.5a/b); SR- $W W$ targets chargino pair production with subsequent decays into $W$ bosons (Figure 3.5c); and SR-Zjets targets chargino-neutralino production with $Z$ and $W$ decays (Figure 3.5d).

Of the three SR categories, the optimization of SR- $m_{\mathrm{T} 2}$ and SR-Zjets were independently conducted by the UCI group ${ }^{1}$ and the Stockholm group ${ }^{2}$, respectively, and therefore the optimization procedure for these SR will not be discussed here. The optimization of SR- $W W$ was conducted jointly by the Harvard group ${ }^{3}$, the CPPM group $^{4}$ and the Carleton group, and involves several components that are presented

\footnotetext{
${ }^{1}$ University of California Irvine, USA

${ }^{2}$ Stockholms Universitet, Sweden

${ }^{3}$ Harvard University, USA

${ }^{4}$ Centre de Physique des Particule de Marseille, France
} 
in detail in the next subsections.

\subsection{Optimization of SR- $W W$}

The optimization of SR- $W W$ was performed using a grid of separate Simplified Model signal samples with the specific topology of Figure 3.5c, with different gaugino mass configurations as listed in Table 4.3. All of the SM backgrounds listed in Section 3.5 are used for the background estimation, with the dominant backgrounds being the $W W$ and the top processes. As explained in Section 4.3, the ATLAS collision data are blinded, and therefore only MC events are used for both SM background and SUSY signal expectations. An integrated luminosity of $\mathcal{L}=21 \mathrm{fb}^{-1}$ at $\sqrt{s}=8 \mathrm{TeV}$ is assumed for the optimization.

The definition of SR-WW is designed and optimized based on a preselection of events. The preselection is designed to remove events that do not follow the general topology and final states of the signals as shown in Figure 3.5c, and its selection criteria are (refer to Section 4.2 for definitions of objects):

- Exactly two oppositely charged leptons with the transverse momentum $p_{\mathrm{T}}>$ $35 \mathrm{GeV}$ and $p_{\mathrm{T}}>20 \mathrm{GeV}$ for leading and subleading leptons, respectively. This ensures that the leptons are from a good hard-scattered event.

- Ignore events with at least one central light jet, forward jet, or $b$-tagged jet. Presence of any jet objects indicate the underlying process does not follow the topology of interest.

- Ignore events with a dilepton invariant mass $m_{\ell \ell}$ within $10 \mathrm{GeV}$ of the $Z$ boson mass (only for ee and $\mu \mu$ channels) 


\subsubsection{Kinematic Variable Choices}

Kinematic variables used for the SR- $W W$ optimization were chosen in two steps: the main cut variables that were motivated by the underlying physics and the additional cut variables that may assist in separating signal events from the background. Some kinematic distributions for these variables are presented in Section 6.2.

\section{Choice of Main Cut Variables}

The physical motivation for the SR- $W W$ is twofold:

1. $H \rightarrow W W$-like category: This category is inspired by the selection used in the ATLAS SM $H \rightarrow W W$ analysis [78] due to the similiarity of the signal topology as well as background composition between the two analyses (see Figure 5.1 for the comparison of the topology). This category targets the physics phasespace where the mass splitting of the chargino and neutralino is near the $W$ boson mass such that the $W$ bosons are likely produced on-shell. The principal kinematic variables used are $p_{\mathrm{T}, \ell \ell}$ and $E_{\mathrm{T}}^{\text {miss,rel }}$, where $p_{\mathrm{T}, \ell \ell}$ is the transverse momentum of the two-lepton system, and $E_{\mathrm{T}}^{\mathrm{miss}, \mathrm{rel}}$ is defined as:

$$
E_{\mathrm{T}}^{\mathrm{miss}, \mathrm{rel}}=\left\{\begin{array}{ll}
E_{\mathrm{T}}^{\mathrm{miss}} & \text { if } \Delta \phi_{\ell, j} \geq \pi / 2 \\
E_{\mathrm{T}}^{\mathrm{miss}} \times \sin \Delta \phi_{\ell, j} & \text { if } \Delta \phi_{\ell, j}<\pi / 2
\end{array},\right.
$$

where $\Delta \phi_{\ell, j}$ is the azimuthal angle between the direction of $\mathbf{p}_{\mathrm{T}}^{\mathrm{miss}}$ and that of the nearest reconstructed object. $E_{\mathrm{T}}^{\text {miss,rel }}$ is effective in suppressing events with mis-measured missing energy due to a nearby object, in which case the $\phi$ angle of $\mathbf{p}_{\mathrm{T}}^{\text {miss }}$ is correlated to that of the mismeasured object.

2. $m_{\mathrm{T} 2}$-region category: This category is inspired by the other SR (SR- $\left.m_{\mathrm{T} 2}\right)$, 


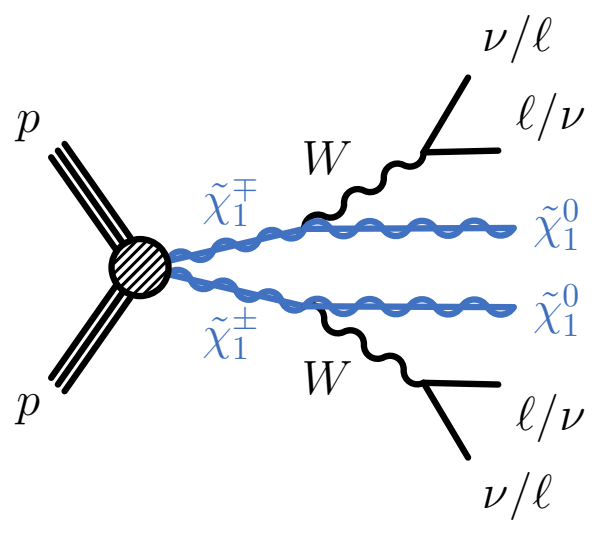

(a)

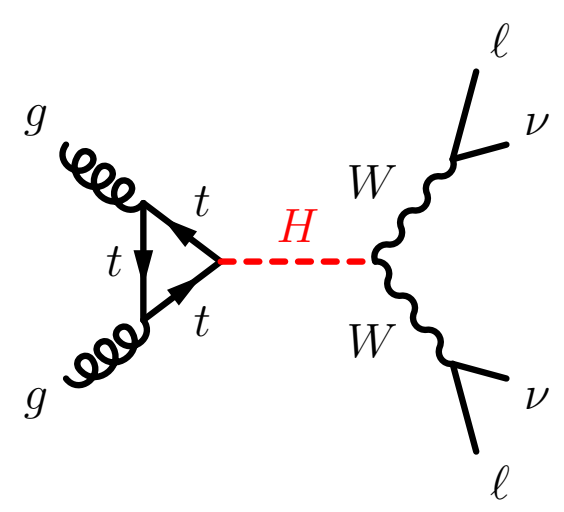

(b)

Figure 5.1: Signal topology for (a) EW SUSY production and (b) SM Higgs production, resulting in the same dilepton final state involving $W$-mediated decays.

which uses a kinematic variable called "stransverse mass" $\left(m_{\mathrm{T} 2}\right)$ [79] that is shown to have strong discriminating power for a signal topology involving a pair of particles that decay to an observable object (such as a lepton) and an unobservable object (such as an LSP). The variable $m_{\mathrm{T} 2}$ is defined as:

$$
m_{\mathrm{T} 2}=\min _{\mathbf{p}_{\mathrm{T}}^{\text {miss,total }}}\left[\max \left(m_{\mathrm{T}}\left(\mathbf{p}_{\mathrm{T}}^{\ell 1}, \mathbf{p}_{\mathrm{T}}^{\mathrm{miss}, \ell 1}\right), m_{\mathrm{T}}\left(\mathbf{p}_{\mathrm{T}}^{\ell 2}, \mathbf{p}_{\mathrm{T}}^{\mathrm{miss}, \ell 2}\right)\right)\right]
$$

where $\mathbf{p}_{\mathrm{T}}^{\text {miss,total }}=\mathbf{p}_{\mathrm{T}}^{\text {miss, } \ell 1}+\mathbf{p}_{\mathrm{T}}^{\text {miss, } \ell 2}$ is the observed missing transverse momentum, $\mathbf{p}_{\mathrm{T}}^{\ell 1}$ and $\mathbf{p}_{\mathrm{T}}^{\ell 2}$ are the transverse momenta of the two leptons, and $\mathbf{p}_{\mathrm{T}}^{\mathrm{miss}, \ell 1}$ and $\mathbf{p}_{\mathrm{T}}^{\text {miss, } \ell 2}$ are the transverse momenta of the unobservable object associated with the respective leptons. The transverse mass variable $m_{\mathrm{T}}[80]$ is defined as:

$$
m_{\mathrm{T}}\left(\mathbf{p}_{\mathrm{T}}, \mathbf{p}_{\mathrm{T}}^{\text {miss }}\right)=\sqrt{2\left(E_{T} E_{\mathrm{T}}^{\text {miss }}-\mathbf{p}_{\mathrm{T}} \cdot \mathbf{p}_{\mathrm{T}}^{\text {miss }}\right)} .
$$




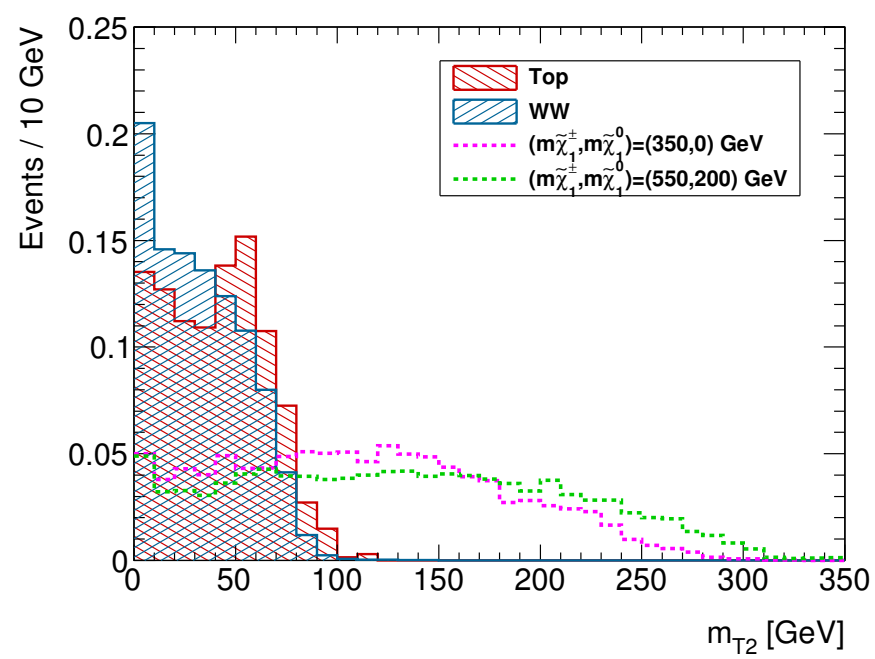

Figure 5.2: Example distribution of $m_{\mathrm{T} 2}$ variable for simulated top, $W W$ and two Simplified Model signals. The top and $W W$ have a cut off at around $W$-boson mass, while the signals have wider distribution extending this cut off.

For SM backgrounds such as $W W$ and $t \bar{t}$ events, the $m_{\mathrm{T} 2}$ distribution has an upper edge at the mass of $W$-boson, while the SUSY signal distribution extends this upper edge due to the missing energy contribution from undetected LSP (see Figure 5.2).

\section{Choice of Additional Cut Variables}

Additional kinematic variables are considered that exploit possible differences in the leptons' behaviour in signal and background events, as well as the correlation between the dilepton system and the missing energy $E_{\mathrm{T}}^{\mathrm{miss}}$. These are:

- Invariant mass of the dilepton system $m_{\ell \ell}$

- Azimuthal angle between the two leptons $\Delta \phi_{\ell, \ell}$

- Transverse momentum of the missing energy and the dilepton system $p_{\mathrm{T}}^{\mathrm{MET}, \ell \ell}$

- Azimuthal angle between the missing transverse momentum and the dilepton system $\Delta \phi_{\mathrm{MET}, \ell \ell}$ 


\subsubsection{Zn as a Figure of Merit}

In high energy physics, an approximation of the significance of signal over background in the form of $N_{s} / \sqrt{N_{b}}$ is widely used, where $N_{s}$ and $N_{b}$ represent the number of signal and background events, respectively (they are obtained from the respective MC samples as listed in Section 4.1.3). This is valid in the case where the expected signal count is much smaller than the expected background (i.e. $N_{s} \ll N_{b}$ ) [81]. However, this approximation no longer holds in the case where both $N_{s}$ and $N_{b}$ suffer from limited statistics. In addition, this ignores uncertainties on the background $\delta_{b}$ which may have drastic effects on the significance.

Due to the above argument, an alternate figure of merit $Z_{n}$ is used to measure the signal significance for this analysis, where $Z_{n}=1.64$ corresponds approximately to $95 \%$ confidence level (CL) for exclusion and a higher $Z_{n}$ value means stronger exclusion power (see Section 6.1.3 for discussion on confidence levels). $Z_{n}$ is expressed as:

$$
Z_{n}=\Phi^{-1}(1-p),
$$

where $\Phi^{-1}$ is the quantile of the standard Gaussian, and $p$ is the cumulative distribution function $(\mathrm{CDF})$ of the binomial distribution, given as:

$$
p\left(N_{s}, N_{b}, \delta_{b}\right)=\sum_{j=N_{s}+N_{b}}^{N_{s}+N_{b}+\frac{1}{\delta_{b}^{2}}} \mathcal{B}\left(j ; N_{s}+N_{b}+\frac{1}{\delta_{b}^{2}}, \frac{1}{1+1 /\left(N_{b} \delta_{b}^{2}\right)}\right) .
$$

The interpretation of Equation 5.5 is the probability of obtaining at least $N_{s}+N_{b}$ 'successful' events in $N_{s}+N_{b}+1 / \delta_{b}^{2}$ random trials, with the probability of success of each trial being $1 /\left(1+1 /\left(N_{b} \delta_{b}^{2}\right)\right)$. This interpretation holds for a background-only 
hypothesis where the observed data match the signal-plus-background expectation, and the uncertainty on the background comes from statistical fluctuation in the control region (CR) estimation [82].

For this optimization study, a constant uncertainty on the background $\delta_{b}=15 \%$ is used. This value is an approximate total uncertainty on the background from a study done in Reference [76]. The impact of this uncertainty on the significance is explored in Section 5.2.

\subsubsection{Cut Value Scan}

For each of the kinematic variables selected in Section $5.1 .1\left(p_{\mathrm{T}, \ell \ell}, E_{\mathrm{T}}^{\mathrm{miss}, \mathrm{rel}}, m_{\mathrm{T} 2}, m_{\ell \ell}\right.$, $\Delta \phi_{\ell, \ell}, p_{\mathrm{T}}^{\mathrm{MET}, \ell \ell}$, and $\left.\Delta \phi_{\mathrm{MET}, \ell \ell}\right)$, a systematic scan of cut values was performed in order to find the values that give the best significance using $Z_{n}$ as defined by Equation 5.4. This is accomplished by estimating $N_{s}$ and $N_{b}$ for each cut requirement for the grid of signal models, and identifing the cut that improves the significance in the majority of the signal points.

\section{Main Variable Cut Scan}

For the SM $H \rightarrow W W$-like category, identical lower cuts on $p_{\mathrm{T}, \ell \ell}$ and $E_{\mathrm{T}}^{\text {miss,rel }}$ were scanned from $50 \mathrm{GeV}$ to $150 \mathrm{GeV}$ in increments of $10 \mathrm{GeV}$. Figure 5.3 shows the optimal cut value (top) for each signal point. The corresponding $Z_{n}$ value is given for $e \mu$ channel only (middle) and combined $e e / e \mu / \mu \mu$ channel (bottom). The combined $Z_{n}$ is calculated as $Z_{n}^{\text {comb }}=\sqrt{Z_{n, e e}^{2}+Z_{n, e \mu}^{2}+Z_{n, \mu \mu}^{2}}$.

For the $m_{\mathrm{T} 2}$-region category, the lower cut on $m_{\mathrm{T} 2}$ was scanned from $60 \mathrm{GeV}$ to $110 \mathrm{GeV}$ in increments of $5 \mathrm{GeV}$. Figure 5.4 shows the optimal cut value (top) for each signal point. The corresponding $Z_{n}$ value is given for $e \mu$ channel only (middle) 
and combined $e e / e \mu / \mu \mu$ channel (bottom).

By comparing the significance map for the $H \rightarrow W W$-like and $m_{\mathrm{T} 2}$-region categories (bottom plots of Figures 5.3 and 5.4, where the red regions indicate higher sensitivity), one can see that $H \rightarrow W W$-like category has overall better sensitivity at lower $\tilde{\chi}_{1}^{ \pm}$mass region, while $m_{\mathrm{T} 2}$-region category has better sensitivity elsewhere. In order to have good sensitivity across the whole mass plane, three sub-SR are defined: SR-WWa to target the low $\tilde{\chi}_{1}^{ \pm}$mass region $\left(m\left(\tilde{\chi}_{1}^{ \pm}\right)<130 \mathrm{GeV}\right)$, with a lower identical

cut of $p_{\mathrm{T}, \ell \ell}$ and $E_{\mathrm{T}}^{\mathrm{miss}, \mathrm{rel}}$ placed at $70 \mathrm{GeV}$; SR- $W W \mathrm{~b}$ to target the middle $\tilde{\chi}_{1}^{ \pm}$mass region $\left(130 \mathrm{GeV}<m\left(\tilde{\chi}_{1}^{ \pm}\right)<180 \mathrm{GeV}\right)$, with a lower $m_{\mathrm{T} 2}$ cut of $90 \mathrm{GeV}$; and SR$W W$ c to target the high $\tilde{\chi}_{1}^{ \pm}$mass region $\left(m\left(\tilde{\chi}_{1}^{ \pm}\right)>180 \mathrm{GeV}\right)$, with a lower $m_{\mathrm{T} 2}$ cut of $100 \mathrm{GeV}$. The proposed cut values correspond to the values that give the best sensitivity in the region of interest (see Figures 5.3 (top) and 5.4 (top)).

\section{Additional Variables Cut Scan}

Additional kinematic variables $\left(m_{\ell \ell}, \Delta \phi_{\ell, \ell}, p_{\mathrm{T}}^{\mathrm{MET}, \ell \ell}\right.$, and $\left.\Delta \phi_{\mathrm{MET}, \ell \ell}\right)$ are scanned for possible improvements. For each variable, a separate scan was performed for each sub-SR (SR- $W W \mathrm{a}, \mathrm{b}, \mathrm{c})$, in the three different lepton flavour combinations $(e e, e \mu, \mu \mu)$ in order to identify the optimal cut values.

Figures 5.5 to 5.8 show the results of the scans (only the $e \mu$ channel is shown for illustrative purpose). For all plots, each bin along the $x$-axis shows the distributions of the ratio of significance $Z_{n}$ compared to the nominal value (without the cut requirement) for each signal points considered. Therefore the first bin is always equal to unity with no width.

Figure 5.5 shows the result of the scan of the $m_{\ell \ell}$ variable. The scan reveals that the upper cut of $120 \mathrm{GeV}$ and $170 \mathrm{GeV}$ improves the overall significance by $\sim 10 \%$ 

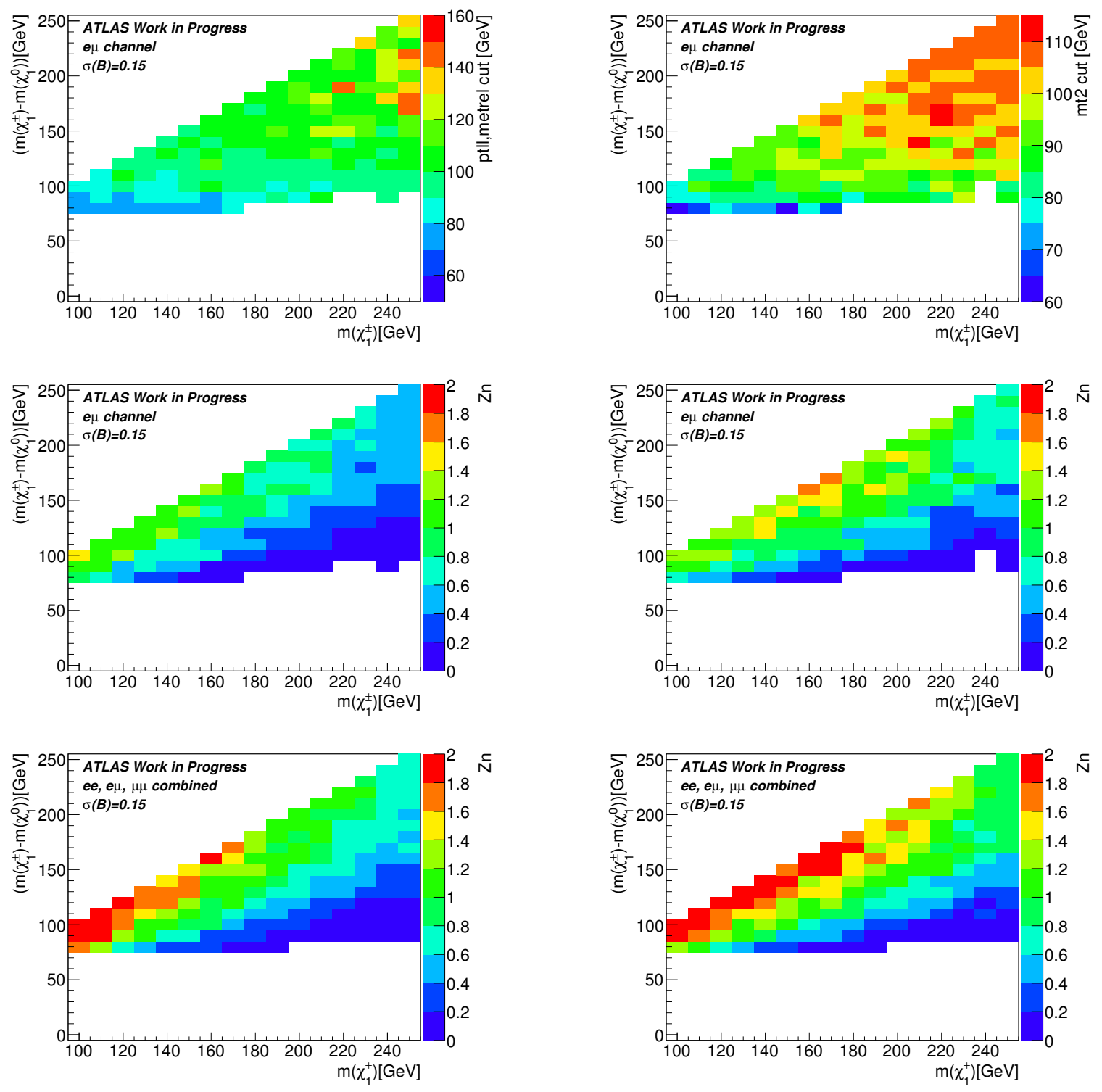

Figure 5.3: The optimal cut value on $p_{\mathrm{T}, \ell \ell}$ and $E_{\mathrm{T}}^{\text {miss,rel }}$ (top) resulting in the

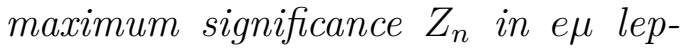
ton flavour combination (middle) and combined flavour channel (bottom) for the $H \rightarrow W W$-like category.

Figure 5.4: The optimal cut value on $m_{\mathrm{T} 2}$ (top) resulting in the maximum significance $Z_{n}$ in e $\mu$ lepton flavour combination (middle) and combined flavour channel (bottom) for the $m_{\mathrm{T} 2}-$ region category. 
to $20 \%$ for SR- $W W$ a and SR- $W W \mathrm{~b}$, respectively. The same cut requirements were chosen for all lepton flavour combinations for simplicity, since the distributions of $Z_{n}$ are in general similar across the three channels. In SR- $W W$ c region, no improvement on the significance was found.

Figures 5.6, 5.7, and 5.8 show the results of the scans of $\Delta \phi_{\ell, \ell}, p_{\mathrm{T}}^{\mathrm{MET}, \ell \ell}$, and

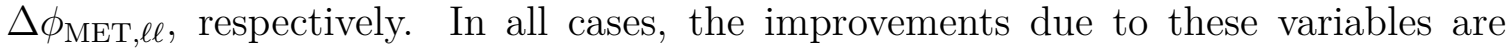
minimal or non-existent, so no cut requirements are imposed for these variables. 

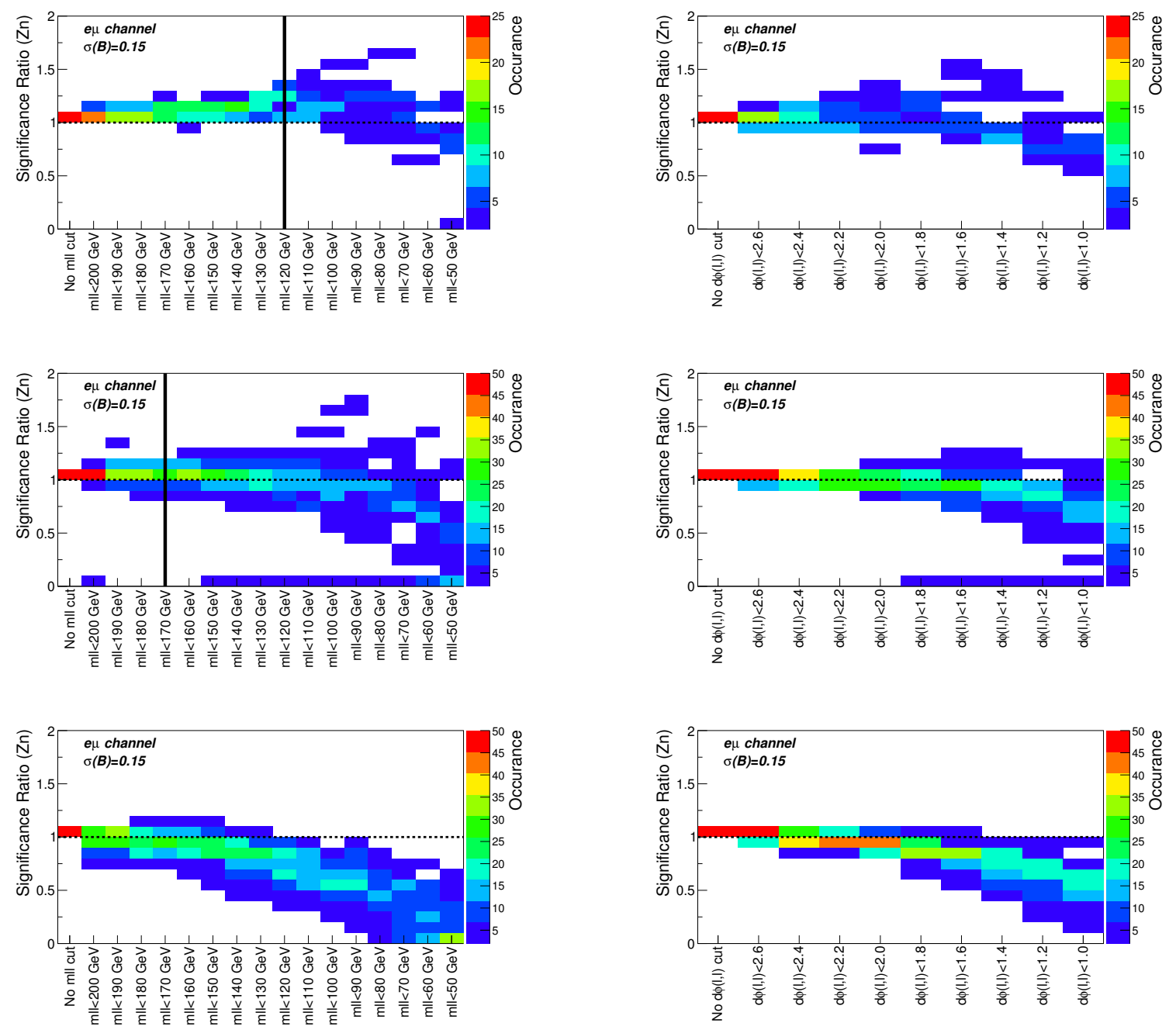

Figure 5.5: Scan of optimal $m_{\ell \ell}$ cut values for $S R-W W$ a (top), $S R-W W b$ (middle) and $S R-W W c$ (bottom). For each cut value (bins along the $x$-axis), the "Occurance" refers to the number of signal grid points falling within the $Z_{n}$ bin (along the $y$-axis). The significance ratio is the ratio of $Z_{n}\left(m_{\ell \ell}\right.$ cut) over $Z_{n}$ (no $m_{\ell \ell}$ cut). Only the e $\mu$ flavour channel is shown for illustrative purpose.

Figure 5.6: Scan of optimal $\Delta \phi_{\ell, \ell}$ cut values for $S R-W W a$ (top), $S R$ $W W b$ (middle) and $S R-W W c$ (bottom). For each cut value (bins along the $x$-axis), the "Occurance" refers to the number of signal grid points falling within the $Z_{n}$ bin (along the y-axis). The significance ratio is the ratio of $Z_{n}\left(\Delta \phi_{\ell, \ell}\right.$ cut) over $Z_{n}\left(\right.$ no $\Delta \phi_{\ell, \ell}$ cut). Only the e $\mu$ flavour channel is shown for illustrative purpose. 

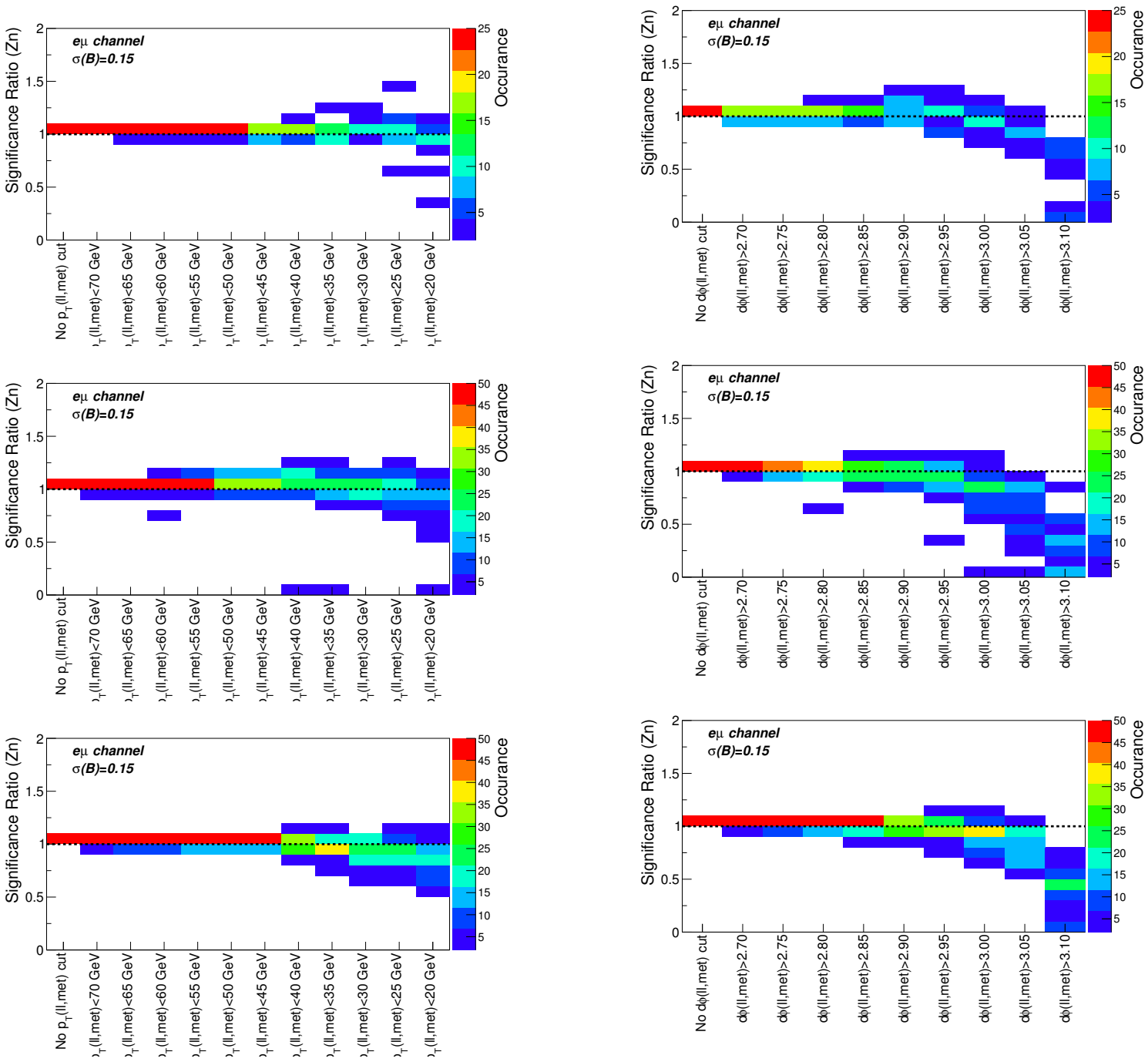

Figure 5.7: Scan of optimal $p_{\mathrm{T}}^{\mathrm{MET}, \ell \ell}$ cut values for $S R-W W a$ (top), SR$W W b$ (middle) and $S R-W W c$ (bottom). For each cut value (bins along the $x$-axis), the "Occurance" refers to the number of signal grid points falling within the $Z_{n}$ bin (along the y-axis). The significance ratio is the ratio of $Z_{n}\left(p_{\mathrm{T}}^{\mathrm{MET}, \ell \ell} \quad c u t\right)$ over $Z_{n}\left(\right.$ no $p_{\mathrm{T}}^{\mathrm{MET}, \ell \ell}$ cut). Only the e $\mu$ flavour channel is shown for illustrative purpose.

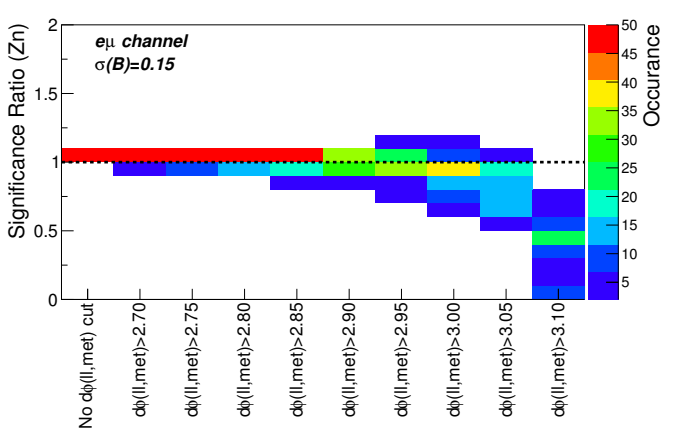

Figure 5.8: Scan of optimal $\triangle \phi_{\mathrm{MET}, \ell \ell}$ cut values for $S R$-WWa (top), $S R-W W b$ (middle) and $S R$ $W W c$ (bottom). For each cut value (bins along the $x$-axis), the "Occurance" refers to the number of signal grid points falling within the $Z_{n}$ bin (along the $y$-axis). The significance ratio is the ratio of $Z_{n}\left(\Delta \phi_{\mathrm{MET}, \ell \mathrm{le}}\right.$ cut $)$ over $Z_{n}$ (no $\Delta \phi_{\text {MET,el }}$ cut). Only the e $\mu$ flavour channel is shown for illustrative purpose. 


\subsection{Impact of Systematic Uncertainties on the Ex- pected Sensitivity}

The optimization study described in Section 5.1 was completed under the assumption that the total uncertainty on the background is constant at $15 \%$ (descriptions of individual systematic uncertainties were given in Section 4.4). It was also assumed that the MC perfectly predicts each of the background components (i.e. no scale factors correcting for the observed discrepancies between data and $\mathrm{MC}$ were applied). However, from the study performed in Reference [76], the total background uncertainties for SR- $W W \mathrm{a} / \mathrm{b} / \mathrm{c}$ were found to be $12 \%, 17 \%$, and $20 \%$ respectively. In the same study, the background due to the SM $W W$ process had a data-to-MC scale factor of 1.2 while other SM backgrounds had scale factors of 1.0. For the purpose of the baseline comparison, these factors were taken into account, and the sensitivity was recalculated.

The results can be seen in Figure 5.9. An overall slight decrease in significance is seen in most regions, due to the higher background uncertainties for $\mathrm{SR}-W W \mathrm{~b}$ and SR- $W W$ c regions. As well, the $W W$ scale factor slightly increases the background estimate $N_{b}$. However, a slight improvement in significance is observed for low $\tilde{\chi}_{1}^{ \pm}$ mass region, where $\mathrm{SR}-W W$ a is expected to have the best performance. Figure $5.9 \mathrm{c}$ depicts the impact on the result of these shifts in the significance.

Figure 5.10 shows the approximation of $95 \% \mathrm{CL}$ on the cross section for the SR$W W$ regions for the case $\mathrm{m}\left(\tilde{\chi}_{1}^{0}\right)=0 \mathrm{GeV}$. The approximation was done by calculating $2 / Z_{n}$, which is a conservative evaluation on the upper limit $\left(Z_{n}=1.64\right.$ corresponds to $95 \% \mathrm{CL})$. The potential to exclude $\mathrm{m}\left(\tilde{\chi}_{1}^{ \pm}\right)<180 \mathrm{GeV}$ can be seen, with the most 


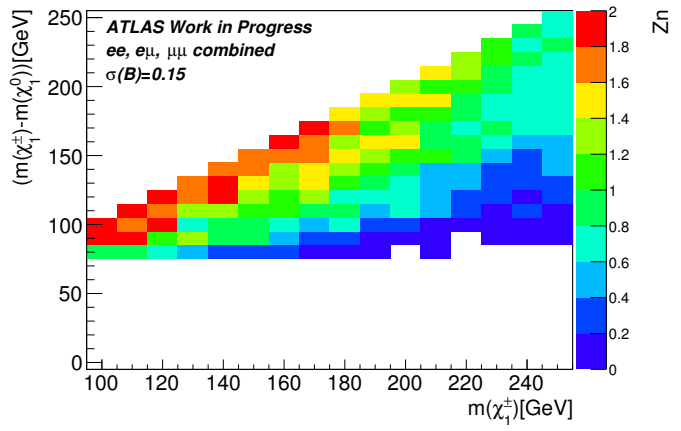

(a)

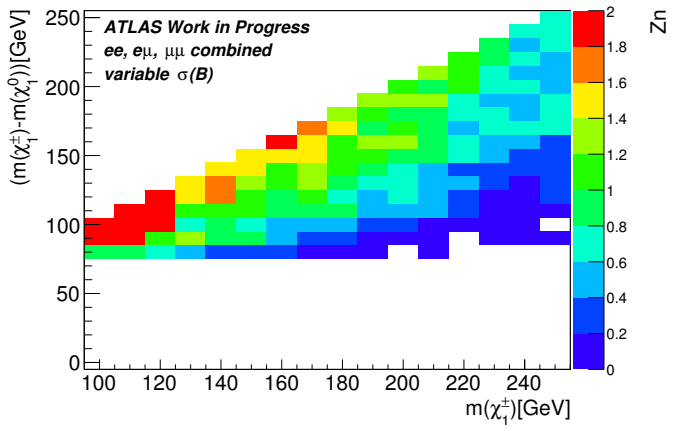

(b)

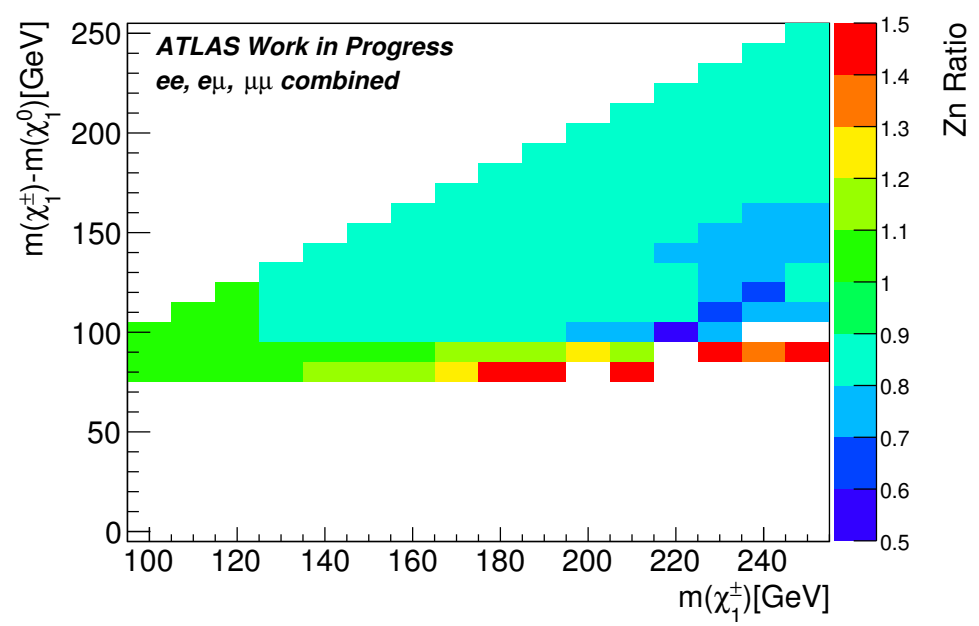

(c)

Figure 5.9: $\quad Z_{n}$ significance of the signal models considered for the optimization for the ee, $\mu \mu$, e $\mu$ combined channel. Constant background uncertainty of $15 \%$ and no scale factor $(S F)$ is applied for (a), while variable uncertainty values described in the text and SF was applied for (b). The shift in $Z_{n}$, expressed as a ratio $(b) /(a)$, is shown in (c).

sensitive point being $\mathrm{m}\left(\tilde{\chi}_{1}^{ \pm}\right)=100 \mathrm{GeV}$. 


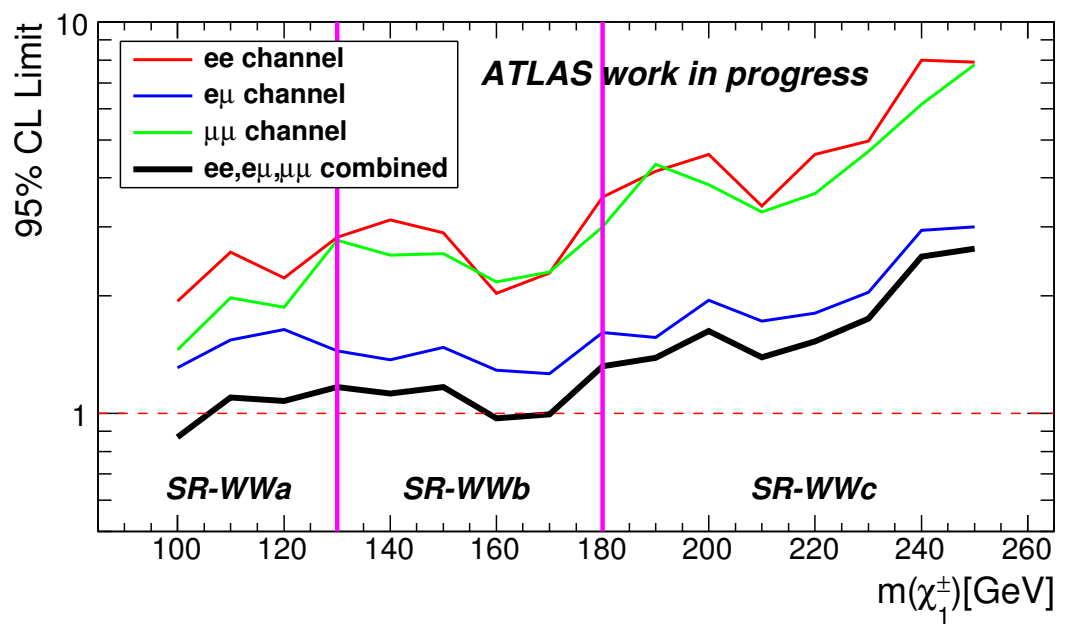

(a)

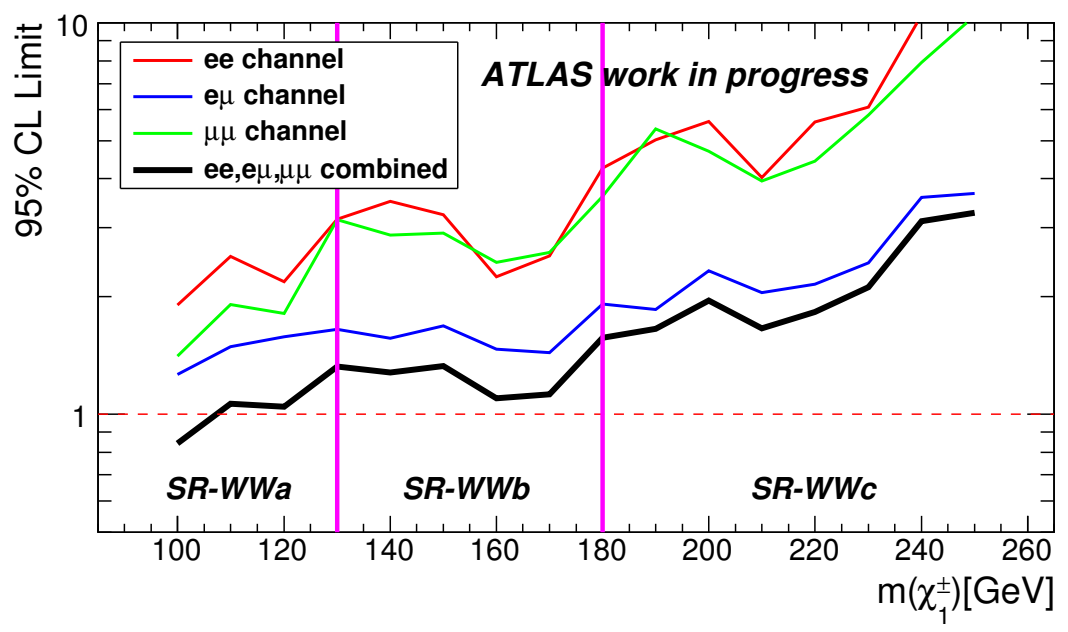

(b)

Figure 5.10: Approximation of 95\% confidence limit on the cross section of a new physical process compared to the SM cross section (y-axis) as a function of $m\left(\tilde{\chi}_{1}^{ \pm}\right)$ for the case $m\left(\tilde{\chi}_{1}^{0}\right)=0 \mathrm{GeV}$. The approximation was done by calculating $2 / Z_{n}$. The overlaid boundaries indicate regions where the three $S R$-WW subregions are expected to perform the best. A total uncertainty of $15 \%$ is assumed for background estimate, and no scale factors (SF) are applied for (a) and variable background uncertainty and SF are applied for (b). 


\begin{tabular}{|c|c|c|c|c|c|}
\hline \multirow[t]{2}{*}{ Signal region } & \multicolumn{3}{|c|}{ SR- $W W$} & \multirow{2}{*}{$\begin{array}{c}\mathrm{SR}-m_{\mathrm{T} 2} \\
\mathrm{a} / \mathrm{b} / \mathrm{c}\end{array}$} & \multirow[t]{2}{*}{ SR-Zjets } \\
\hline & $\mathrm{a}$ & $\mathrm{b}$ & c & & \\
\hline $\begin{array}{r}\text { lepton flavour } \\
\text { lepton } p_{T}[\mathrm{GeV}]^{5} \\
\left|m_{\ell \ell}-m_{Z}\right|^{6}\end{array}$ & \multicolumn{4}{|c|}{$\begin{aligned} & e e, \mu \mu, e \mu \\
> & (35,20) \\
& >10\end{aligned}$} & $\begin{aligned} & e e, \mu \mu \\
> & (35,20) \\
& <10\end{aligned}$ \\
\hline $\begin{array}{r}\text { central light jets } \\
\text { signal } b \text {-jets } \\
\text { signal forward jets }\end{array}$ & \multicolumn{4}{|c|}{$\begin{array}{l}=0 \\
=0 \\
=0\end{array}$} & $\begin{array}{l}\geq 2 \\
=0 \\
=0\end{array}$ \\
\hline $\bar{E}_{\mathrm{T}}^{\text {miss,rel }}[\mathrm{GeV}]$ & $>80$ & - & - & - & $>80$ \\
\hline$p_{T, l l}[\mathrm{GeV}]$ & $>80$ & - & - & - & $>80$ \\
\hline$m_{\mathrm{T} 2}[\mathrm{GeV}]$ & - & $>90$ & $>100$ & $>90 / 120 / 150$ & - \\
\hline$m_{\ell \ell}[\mathrm{GeV}]$ & $<120$ & $<170$ & - & - & - \\
\hline$\Delta R_{\ell \ell}{ }^{7}$ & - & - & - & - & {$[0.3,1.5]$} \\
\hline$m_{j j}[\mathrm{GeV}]$ & - & - & - & - & {$[50,100]$} \\
\hline
\end{tabular}

Table 5.1: Summary of $S R-m_{\mathrm{T} 2}, S R-W W$ and $S R-Z j$ jets signal region definitions.

\subsection{Summary of the Signal Regions}

Independent optimization studies were done on the three SR (SR- $W W, \mathrm{SR}-m_{\mathrm{T} 2}$, and SR-Zjets) for this analysis. The final SR definitions are summarized in Table 5.1.

Note that SR-Zjets focuses on topology involving final-state quarks (see Figure $3.5 \mathrm{~d}$ ), and therefore require the selection of at least 2 central light jets, as well as a cut on jet kinematic variables such as dijet invariant mass $m_{j j}$.

\footnotetext{
${ }^{5}\left(p_{T}^{\ell 1}, p_{T}^{\ell 2}\right)$

${ }^{6}$ Applied for $e e, \mu \mu$ only

${ }^{7} \Delta R_{\ell \ell}=\sqrt{\Delta \eta_{\ell \ell}^{2}+\Delta \phi_{\ell \ell}^{2}}$
} 


\section{RESULTS AND INTERPRETATION}

In Chapter 4, general methodology to search for EW SUSY signatures with 2 finalstate leptons was outlined. This included definitions of relevant objects, criteria for event selection in order to identify such processes, introduction of data and Monte-Carlo (MC) samples used to model the SM and SUSY processes, as well as a description of systematic uncertainties. In Chapter 5, the optimization process of SR was detailed and it defined seven SR for this analysis that will maximize the chance to observe signal events, if they exist.

In this chapter, results from the analysis [28] for which I was personally responsible are presented. Firstly, some key probability and statistical tools are reviewed. Secondly, selected kinematic distributions in various regions, as well as some visualizations of candidate signal events are shown. Results from the three different fitting procedures (background-only, model-independent and model-dependent) are subsequently presented. The combination of results with the trilepton analysis concludes this chapter. 


\subsection{Probability and Statistics}

In particle physics (in fact in most natural sciences), statistical analysis plays a very important role in either proving or disproving a theory or a hypothesis. Utilizing statistical techniques, one can test whether observed data are in agreement with the predicted probabilities from an underlying theory, or result from random chance. A statistical test, called hypothesis testing, is widely used to search for new physics beyond the SM at the ATLAS experiment.

\subsubsection{Hypothesis Testing}

A hypothesis refers to a predicted probability distribution of a vector of data $\mathbf{x}$. A null hypothesis, $H_{0}$, is traditionally referred to as a hypothesis under consideration, while an alternate hypothesis, $H_{1}$, is tested against the null hypothesis. These hypotheses will define the probability density functions (pdf), $g\left(\mathbf{x} \mid H_{0}\right)$ and $g\left(\mathbf{x} \mid H_{1}\right)$, respectively.

A test is performed by defining a region of $\mathbf{x}$-space called the critical region, $w$, within a significance level $\alpha$. If the observed data are within this critical region, that is $P\left(\mathbf{x} \in w \mid H_{0}\right) \leq \alpha$, then the null hypothesis $H_{0}$ is rejected in favour for the alternate hypothesis $H_{1}$ (see Figure 6.1 for an example illustration). Often in highenergy physics, $\mathbf{x}$ represents the measured properties of the candidate events with mean $\mu$, and $w$ is defined by the signal region definitions (see Chapter 5). Instead of using the full measurement of $\mathbf{x}$, a test-statistic, $t(\mathbf{x})$, is often used.

Neyman-Pearson lemma states that the ratio of pdf of $H_{0}$ and $H_{1}$ :

$$
\lambda(\mathbf{x})=\frac{g\left(\mathbf{x} \mid H_{1}\right)}{g\left(\mathbf{x} \mid H_{0}\right)}
$$




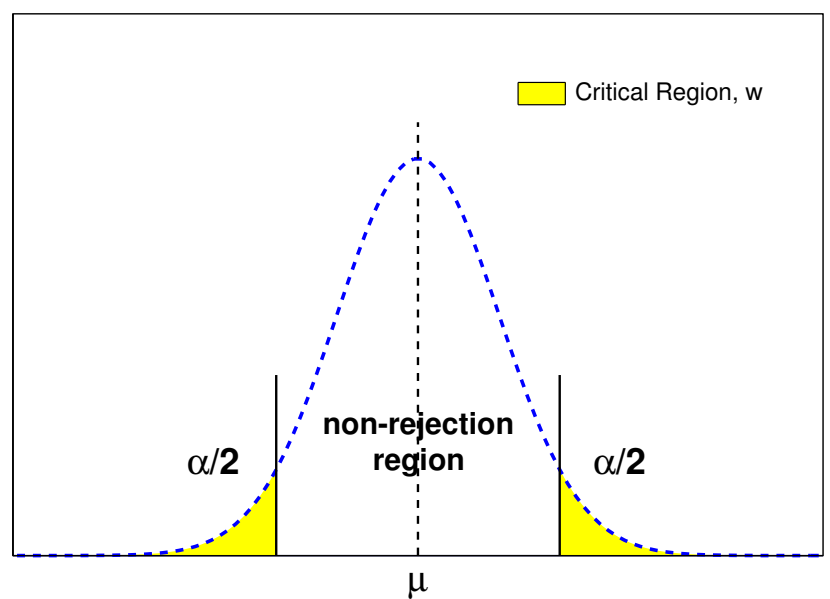

Figure 6.1: An example illustration of a critical region in a simple Gaussian distribution with mean $\mu$. The hypothesis is rejected if observed data are within a significance level $\alpha$ corresponding to the critical region represented in the yellow shaded region.

gives the most powerful test for rejection of the null hypothesis $H_{0}[3,83]$. Equivalently, Equation 6.1 is the optimal test statistic where the critical region $w$ is defined by a single cut on $\lambda$. The likelihood ratio is often used as the ratio of the pdf in Equation 6.1:

$$
Q=\frac{L(n \mid s+b)}{L(n \mid b)}
$$

where $L(n \mid x)$ is the likelihood function $\left(=\frac{(x)^{n}}{n !} e^{-(x)}\right.$ for Poisson distributed $\left.n\right)$, which is the likelihood of observing $n$ events given the hypothetical mean value of $x$. Therefore, Equation 6.2 is the likelihood ratio of hypothesis with existence of SUSY signal, $s$, to the hypothesis with only the SM backgrounds, $b$.

The distribution of a likelihood ratio is often difficult to determine, so it is often modified to a log-likelihood ratio:

$$
t(\mathbf{x})=-2 \ln (Q)
$$


since the minimum of this function is easier to obtain for the case with multiple parameters.

\subsubsection{Profile Likelihood Ratio}

The likelihood ratio (Equation 6.2) disregards any uncertainties associated with the data. However, systematic uncertainties affect the estimation of both background and signal yields, and therefore affect the test statistic introduced above. These systematic uncertainties are incorporated to the test statistic as nuisance parameters $\nu$. The full likelihood function is written as:

$$
L\left(\mu_{s}, \nu\right)=P\left(n \mid \mu_{s} s+\sum_{m}^{N_{b k g}} \mu_{b} b\right) \times N(\tilde{\nu} \mid \nu),
$$

where $\mu_{b}$ is the background normalization factors for each background category to account for the difference in data to $\mathrm{MC}$, and $\mu_{s}$ is the signal strength parameter, with $\mu_{s}=0$ corresponding to the background-only hypothesis and $\mu_{s}=1$ corresponding to the signal+background hypothesis. $N(\tilde{\nu} \mid \nu)$ is the systematic uncertainty pdf which is modelled by Normal distributions as a function of nuisance parameters. These nuisance parameters interfere with the construction of a test statistic, but they can be effectively removed by defining a profile likelihood ratio:

$$
\lambda(\mu)=\frac{L(\mu, \hat{\hat{\nu}}(\mu))}{L(\hat{\mu}, \hat{\nu})}
$$

where the numerator is called the profile likelihood, with $\hat{\hat{\nu}}$ corresponding to the value of $\nu$ that maximizes the likelihood for a given $\mu$, and $\hat{\mu}$ and $\hat{\nu}$ in the denominator are the true maximum likelihood estimators [3]. This is used in place of Equation 6.2 as 
the test statistic. HistFitter [84] is used for the computation, and the version used was HistFitter-00-00-29.

Within the HistFitter environment, the goodness of fit is given by an "estimated distance to minimum" (EDM). A successful fit would have EDM value of $\mathcal{O}\left(10^{-4}\right)$.

\subsubsection{Confidence Levels and the $C L_{s}$ Method}

Confidence levels for excluding the alternate hypothesis (hypothesis that SUSY particle production, $s$, is present along with the SM production, $b$ ) can be determined by finding the $p$-value, $p_{\mu}$ :

$$
p_{\mu}=C L_{s+b}=P\left(t \leq t_{o b s} \mid s+b\right),
$$

where the subscript $\mu$ denotes the hypothesis with the existence of SUSY signal, and the right hand side indicates the probability that the test statistic $t(\mathbf{x})$ is less than the observed data assuming the presence of both SUSY and the SM (the yellow area in Figure 6.2). Correspondingly, the confidence level (CL) for exclusion is equal to $\left(1-C L_{s+b}\right)$. In typical SUSY searches, a model is excluded when $p_{\mu} \leq 5 \%$. This is equivalent to the model being excluded at 95\% CL.

If a simple Poisson statistic is assumed as a test statistic, and in the absence of any systematic uncertainties, this probability is simply the sum of Poisson probabilities (with the subscript obs indicating the observed quantity):

$$
P\left(n \leq n_{o b s} \mid s+b\right)=\sum_{n=0}^{n_{o b s}} \frac{(s+b)^{n}}{n !} e^{-(s+b)} .
$$

The calculation of the confidence level using $C L_{s+b}$ may be erroneous in the case 


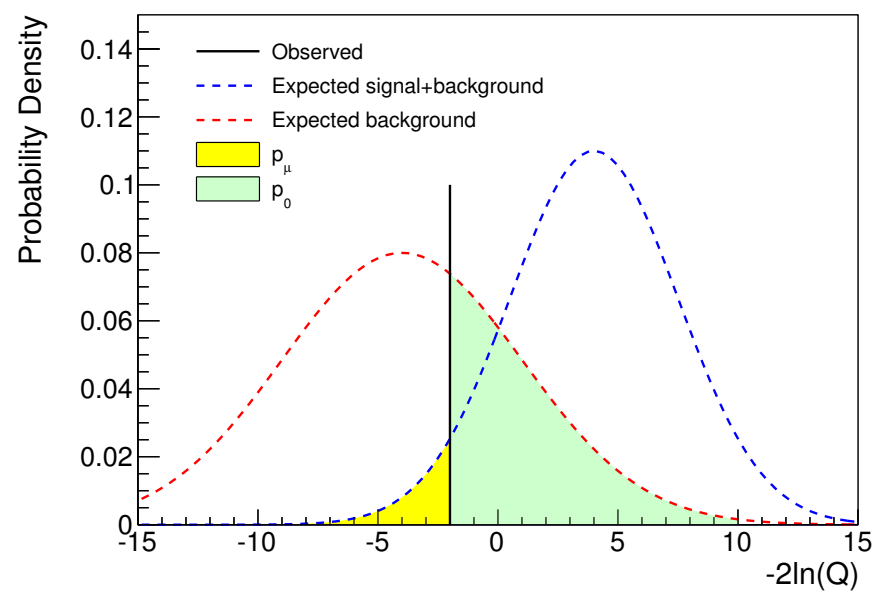

Figure 6.2: Illustration of probability densities. The two distributions represent the pdf of null (red) and alternate (blue) hypothesis, and the black line represent the observed value of the test-statistic. The green (yellow) area corresponds to the p-value of the null (alternate) hypothesis, denoted as $p_{0}\left(p_{\mu}\right)$.

of low sensitivity (i.e. $s<<b$ and the data fluctuate below $b$ ). An example of low sensitivity is if the expected background and signal count are $b=10$ and $s=1$, respectively, and the observed count is $n=8$. This often occurs in SUSY searches, where a broad range of a model's parameter space is to be covered by a small number of SR.

The above can be remedied by penalizing $p_{\mu}$ by an amount that counters the trend of $C L_{s+b}$ :

$$
1-p_{0}=C L_{b}=P\left(t \leq t_{o b s} \mid b\right),
$$

where $p_{0}$ is the $p$-value of the null hypothesis (green area in Figure 6.2). In the simple case of Poisson statistic without any systematic uncertainties, this becomes:

$$
P\left(n \leq n_{o b s} \mid b\right)=\sum_{n=0}^{n_{\text {obs }}} \frac{b^{n}}{n !} e^{-b}
$$


The effective $p$-value of the exclusion is the ratio of Equation 6.6 and Equation 6.8:

$$
C L_{s}=\frac{C L_{s+b}}{C L_{b}}=\frac{p_{\mu}}{1-p_{0}}
$$

This $C L_{s}$ technique is used widely for searches in particle physics, and is used for the exclusion limits in this analysis (see Section 6.5). The computation of $C L_{s}$ is done by HistFitter-00-00-29.

\subsection{Kinematic Distributions in Signal Regions}

As discussed in Chapter 4, one of the major analysis steps is to verify the kinematic distributions of the candidate events in the SR for agreement of data to the MC expectations as well as background compositions.

At the preselection stage, as well as for each SR defined in Chapter $5\left(\mathrm{SR}-m_{\mathrm{T} 2}\right.$, SR- $W W$ and SR-Zjets), some relevant kinematic distributions are shown to depict the agreement of data to the total SM background. Visualization of some candidate events in the SR are also presented in the last subsection.

\subsubsection{Kinematic Distributions in the Preselection Region}

Before inspecting the kinematic distributions in the SR, it is important to verify the properties of the candidate events in the preselection region. The preselection region selects general, two-lepton signal-like signatures and is better suited to evaluate the data to MC agreement due to the larger sample size than in the SR. The definition of a preselection was given in Section 5.1.

Figure 6.3 shows the kinematic distribution of $E_{\mathrm{T}}^{\text {miss,rel }}$ (defined in Equation 5.1) 
and the dilepton invariant mass $m_{\ell \ell}$ for $e e$ and $\mu \mu$ channels. It can be seen that the data (black points) agree within the total uncertainties of the SM background estimated by the MC (filled histograms). In the $m_{\ell \ell}$ distributions, the effect of the $Z$ boson mass veto is clearly visible. In addition, the similarity in the distribution shapes between the ee and $\mu \mu$ channels is evident. This is because the branching ratio for a SUSY or a SM particle to electron and muon are nearly identical (for example, $\mathrm{BR}(W \rightarrow e \nu) / \mathrm{BR}(W \rightarrow \mu \nu)=10.75 \% / 10.57 \%$ and $\operatorname{BR}(Z \rightarrow e e) / \operatorname{BR}(Z \rightarrow \mu \mu)$ $=3.363 \% / 3.366 \%[3])$. For this reason, these two channels will be combined and hereafter referred to as the same-flavour (SF) channel.

Figure 6.4 shows the same kinematic distribution for the $e \mu$ channel (hereafter referred to as the different-flavour, or DF, channel). As shown, the shape and the background compositions of the distribution differ from the SF channel, but good data to MC agreement is observed. 

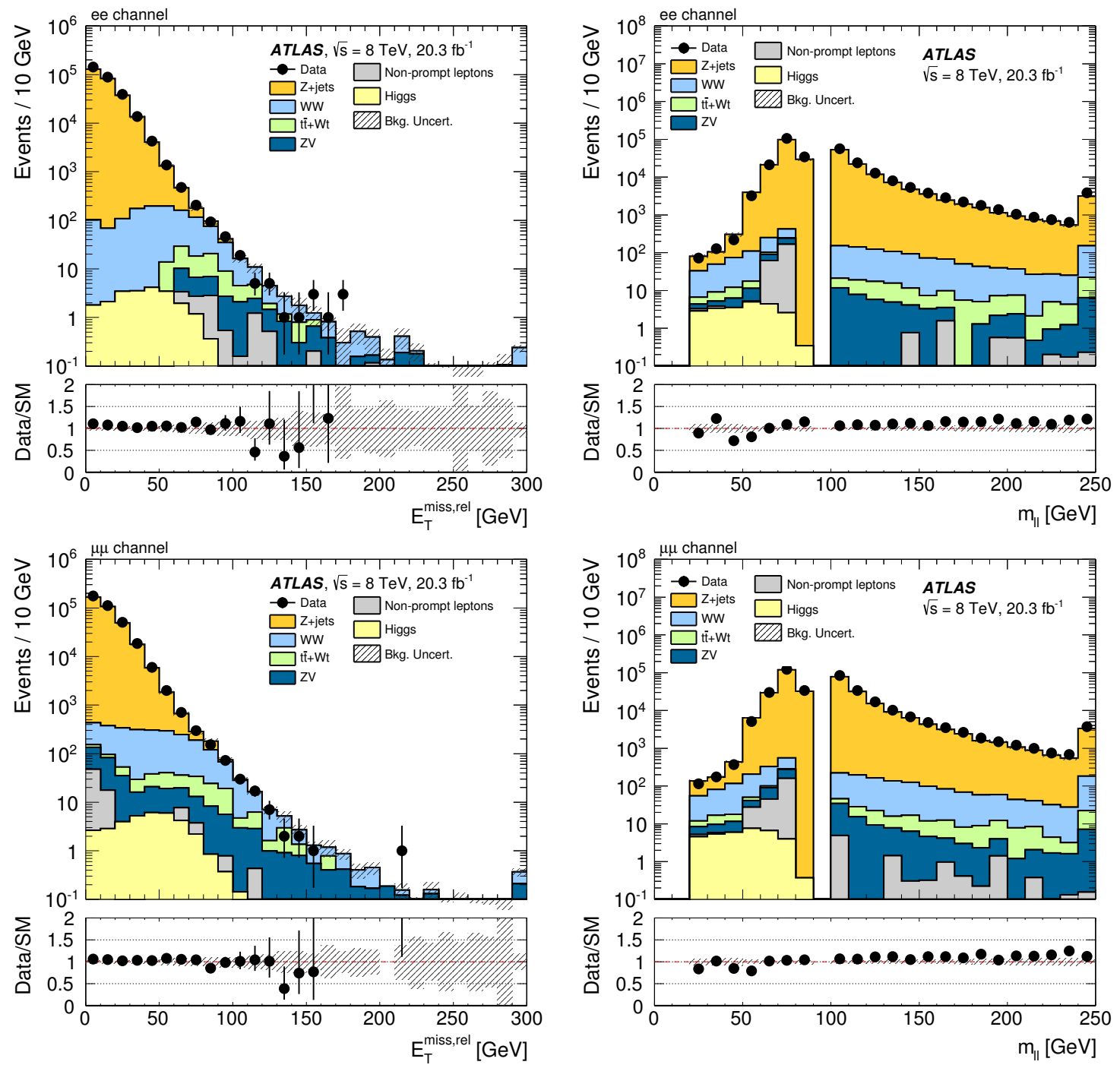

Figure 6.3: Kinematic distributions of $E_{\mathrm{T}}^{\mathrm{miss}, \mathrm{rel}}$ (left) and $m_{\ell \ell}$ (right) in the preselection region for the ee (top) and $\mu \mu$ (bottom) channel. These channels are combined and referred to as the same-flavour (SF) channel. The error bars on the data points are statistical uncertainty, while the hashed region includes all systematic uncertainties described in Section 4.4 . 

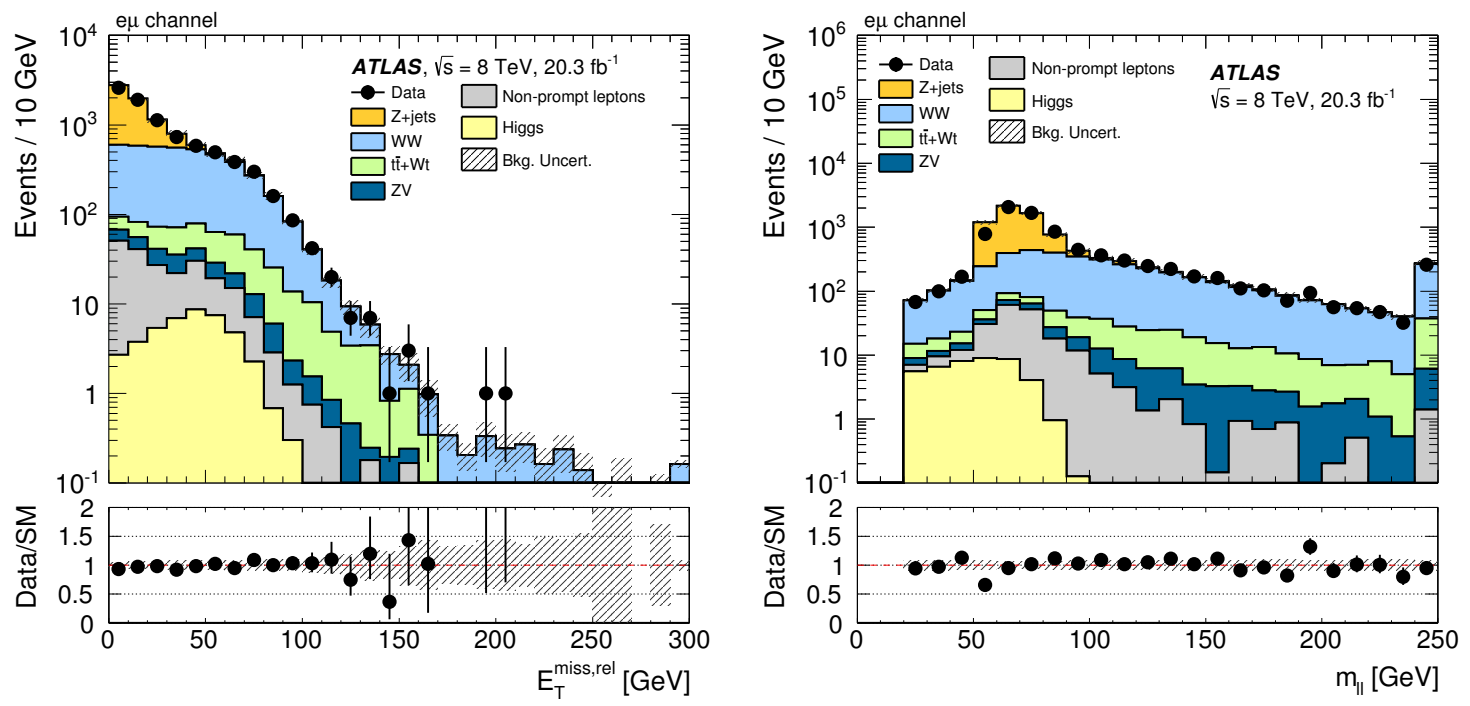

Figure 6.4: Kinematic distributions of $E_{\mathrm{T}}^{\mathrm{miss}, \mathrm{rel}}$ (left) and $m_{\ell \ell}$ (right) in the preselection region for the e $\mu$ channel. This channel is also referred to as the different-flavour (DF) channel. The error bars on the data points are statistical uncertainty, while the hashed region includes all systematic uncertainties described in Section 4.4. 

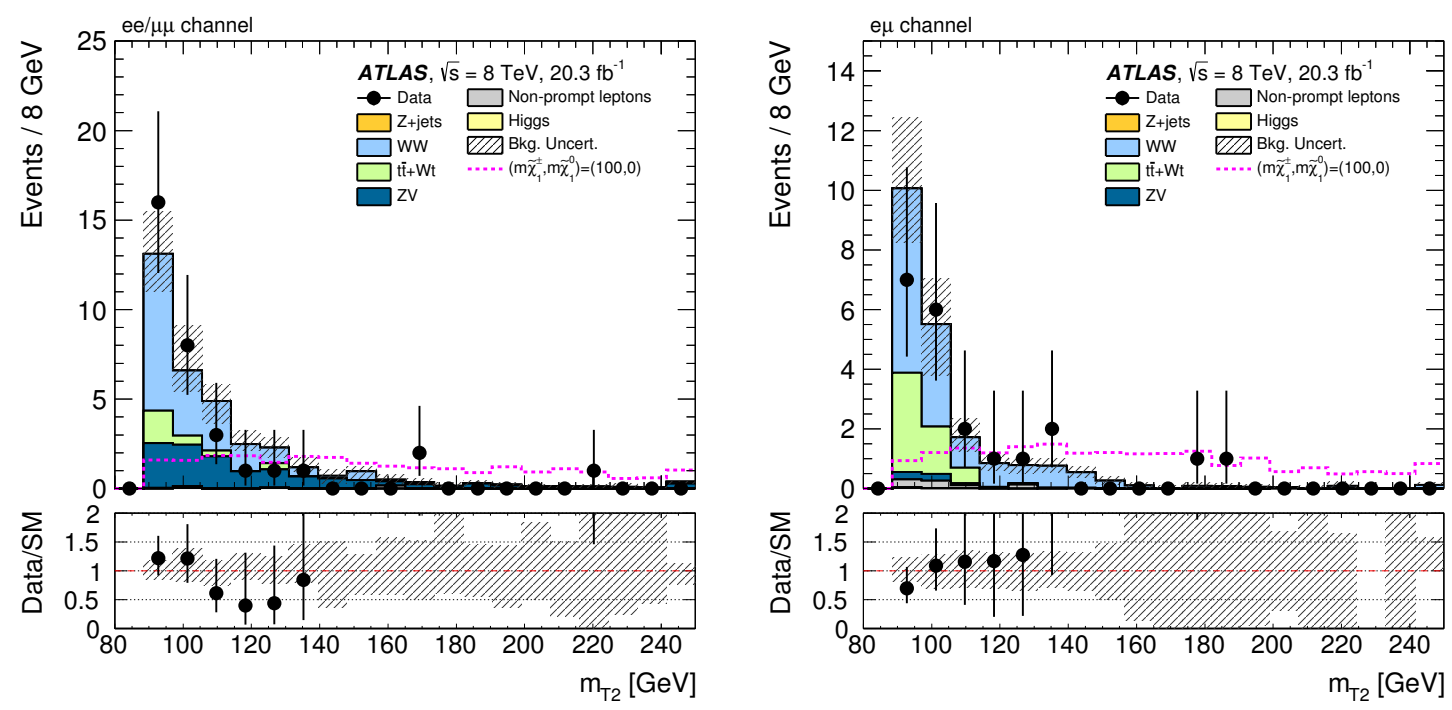

Figure 6.5: Kinematic distributions of $m_{\mathrm{T} 2}$ in the $S R-m_{\mathrm{T} 2}$ a region for the $S F$ channel (left) and the DF channel (right). An example signal point corresponding to the Simplified Model $\tilde{\chi}_{1}^{ \pm} \tilde{\chi}_{1}^{ \pm} \rightarrow \tilde{\ell} \tilde{\ell}$ with $\left(m_{\tilde{\chi}_{1}^{ \pm}}, m_{\tilde{\chi}_{1}^{0}}\right)=(100,0)$ GeV is overlaid.

\subsubsection{Kinematic Distributions in SR- $m_{\mathrm{T} 2}$}

Figures 6.5 through 6.7 show the distribution of the $m_{\mathrm{T} 2}$ variable (defined in Equation 5.2) in the three SR- $m_{\mathrm{T} 2} \mathrm{a} / \mathrm{b} / \mathrm{c}$ regions (summarized in Table 5.1) for the $\mathrm{SF}$ and the DF channels. In all of the plots, an example signal distribution corresponding to the Simplified Model $\tilde{\chi}_{1}^{ \pm} \tilde{\chi}_{1}^{ \pm} \rightarrow \tilde{\ell} \tilde{\ell}$ with $\left(m_{\tilde{\chi}_{1}^{ \pm}}, m_{\tilde{\chi}_{1}^{0}}\right)=(100,0) \mathrm{GeV}$ is overlaid.

It can be seen in all of the plots that the data (in black points) agree with the MC expectations (filled histograms) within the systematic uncertainties, with the exception of the two events at high $m_{\mathrm{T} 2}$ region in the DF channel (the three events in the SF channel are within the systematic uncertainties). Initially, these events seem to agree with the signal distribution, however they are likely statistical fluctuations, the reason being that the signal prediction (dotted histogram) indicates an expected excess of events uniformly for the entire high $m_{\mathrm{T} 2}$ region over the SM, and the observed 

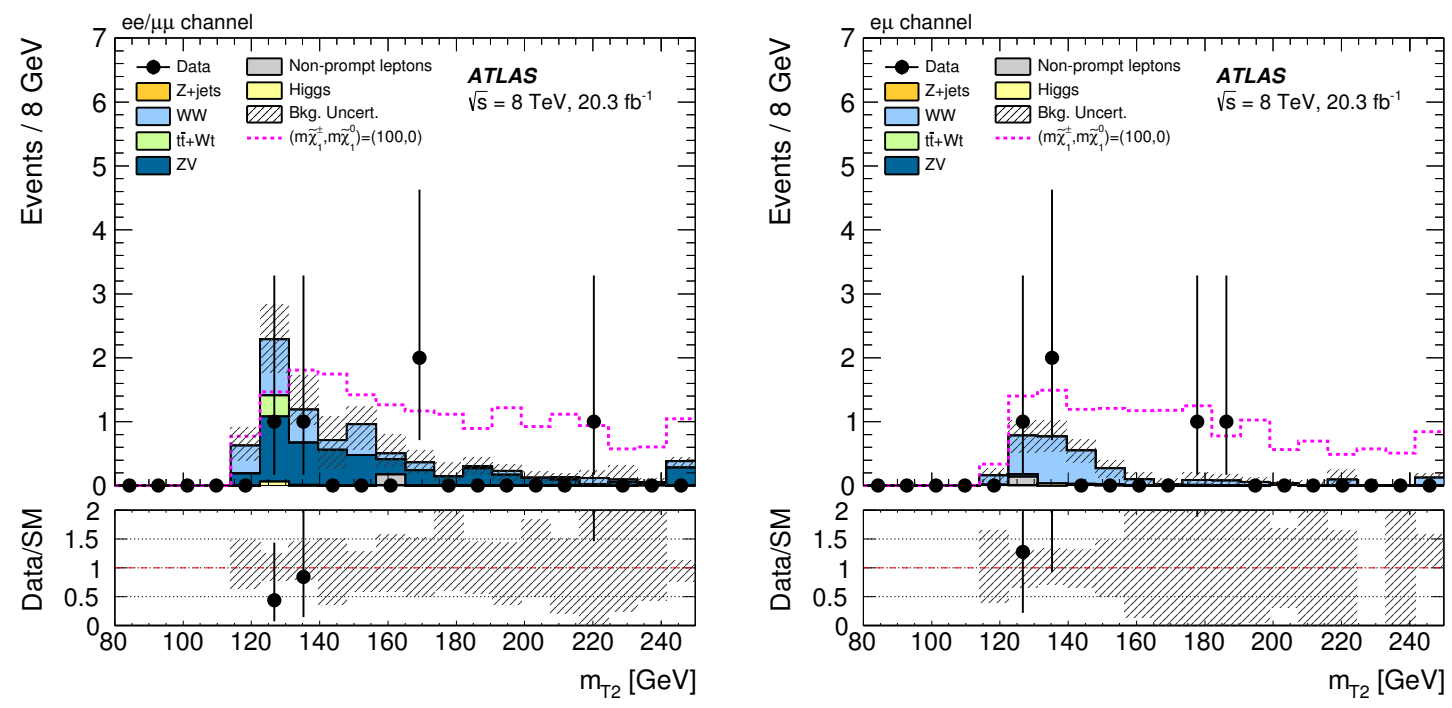

Figure 6.6: Kinematic distributions of $m_{\mathrm{T} 2}$ in the $S R-m_{\mathrm{T} 2} b$ region for the $S F$ channel (left) and the DF channel (right). An example signal point corresponding to the Simplified Model $\tilde{\chi}_{1}^{ \pm} \tilde{\chi}_{1}^{ \pm} \rightarrow \tilde{\ell} \tilde{\ell}$ with $\left(m_{\tilde{\chi}_{1}^{ \pm}}, m_{\tilde{\chi}_{1}^{0}}\right)=(100,0)$ GeV is overlaid.
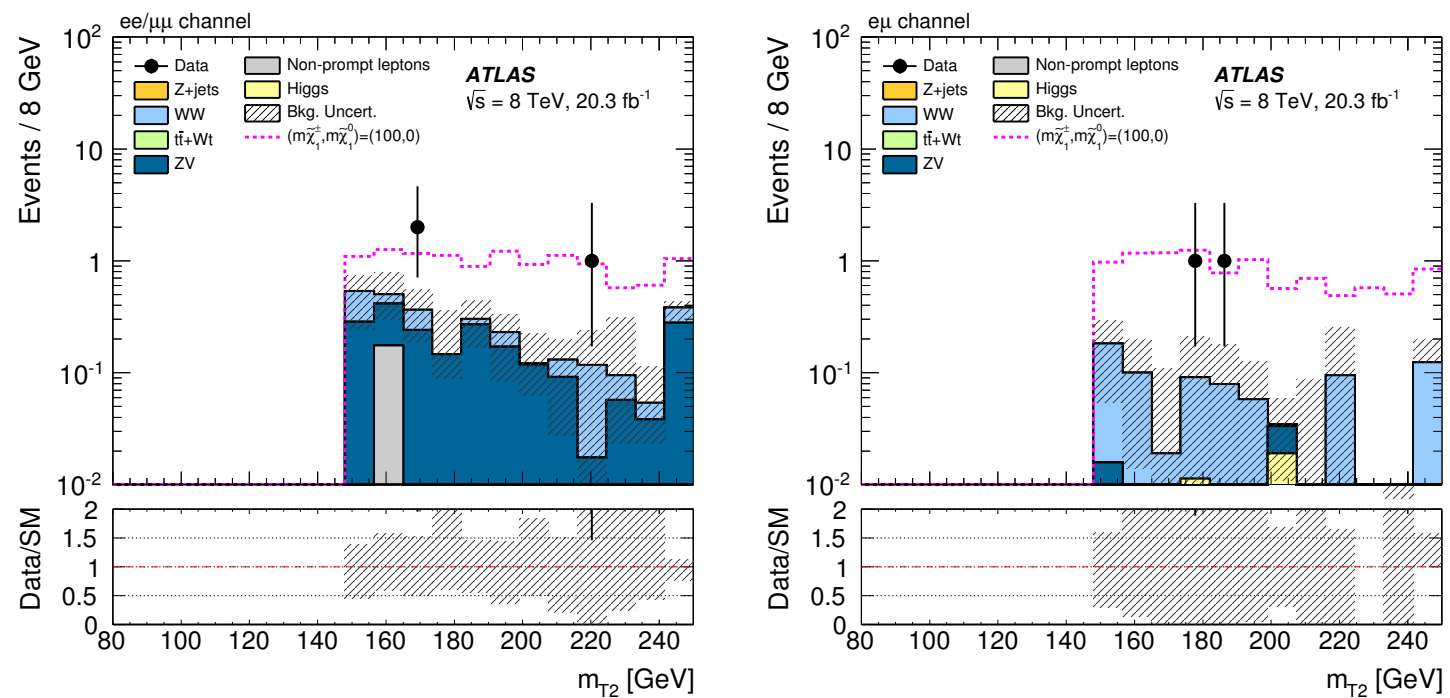

Figure 6.7: Kinematic distributions of $m_{\mathrm{T} 2}$ in the $S R-m_{\mathrm{T} 2}$ c region for the $S F$ channel (left) and the DF channel (right). An example signal point corresponding to the Simplified Model $\tilde{\chi}_{1}^{ \pm} \tilde{\chi}_{1}^{ \pm} \rightarrow \tilde{\ell} \tilde{\ell}$ with $\left(m_{\tilde{\chi}_{1}^{ \pm}}, m_{\tilde{\chi}_{1}^{0}}\right)=(100,0)$ GeV is overlaid.

data seem to contradict this expectation in this SR.

Due to the more stringent selection criteria of SR- $m_{\mathrm{T} 2} \mathrm{c}$, the statistics in this $\mathrm{SR}$ 

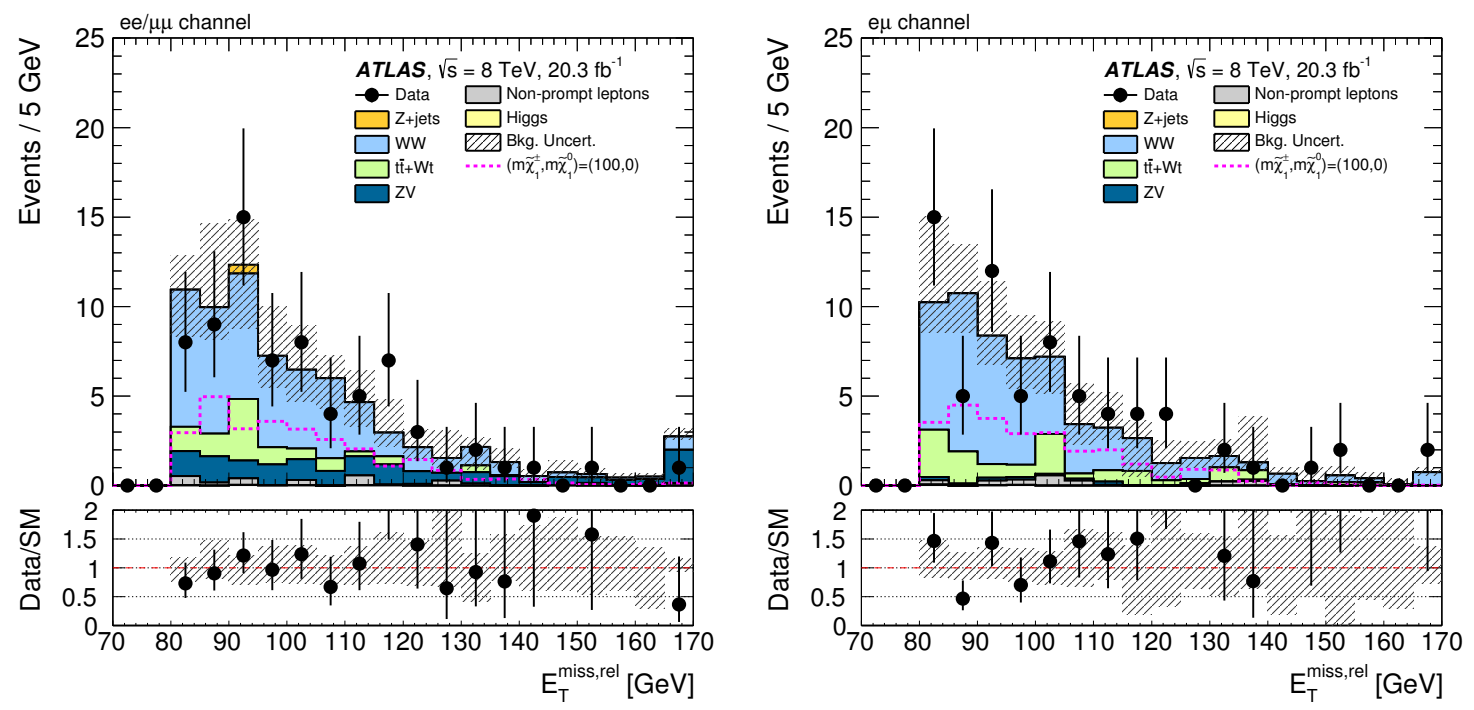

Figure 6.8: Kinematic distributions of $E_{\mathrm{T}}^{\mathrm{miss}, \mathrm{rel}}$ in the $S R$-WWa region for the $S F$ channel (left) and the DF channel (right). An example signal point corresponding to the Simplified Model $\tilde{\chi}_{1}^{ \pm} \tilde{\chi}_{1}^{ \pm} \rightarrow W W$ with $\left(m_{\tilde{\chi}_{1}^{ \pm}}, m_{\tilde{\chi}_{1}^{0}}\right)=(100,0)$ GeV is overlaid.

is poor (observed total 3 events and 2 events in the SF and DF channel, respectively).

Hence the distribution for this region is depicted with a logarithmic scale.

\subsubsection{Kinematic Distributions in SR- $W W$}

Figures 6.8 through 6.10 show the distributions of the $E_{\mathrm{T}}^{\text {miss,rel }}$ variable in the three SR- $W W$ a/b/c regions (summarized in Table 5.1) for the SF and the DF channels. For this SR, an example signal point of the Simplified Model $\tilde{\chi}_{1}^{ \pm} \tilde{\chi}_{1}^{ \pm} \rightarrow W W$ with $\left(m_{\tilde{\chi}_{1}^{ \pm}}, m_{\tilde{\chi}_{1}^{0}}\right)=(100,0) \mathrm{GeV}$ is overlaid.

Similar to the SR- $m_{\mathrm{T} 2}$ regions, the data (in black points) agree with the MC predictions (filled histograms) within the systematic uncertainties. The example signal distribution (dotted histogram) shows similar behaviour to the SM for the $E_{\mathrm{T}}^{\mathrm{miss}, \mathrm{rel}}$ variable in this SR. Hence, to first order, the data appear to agree with the 

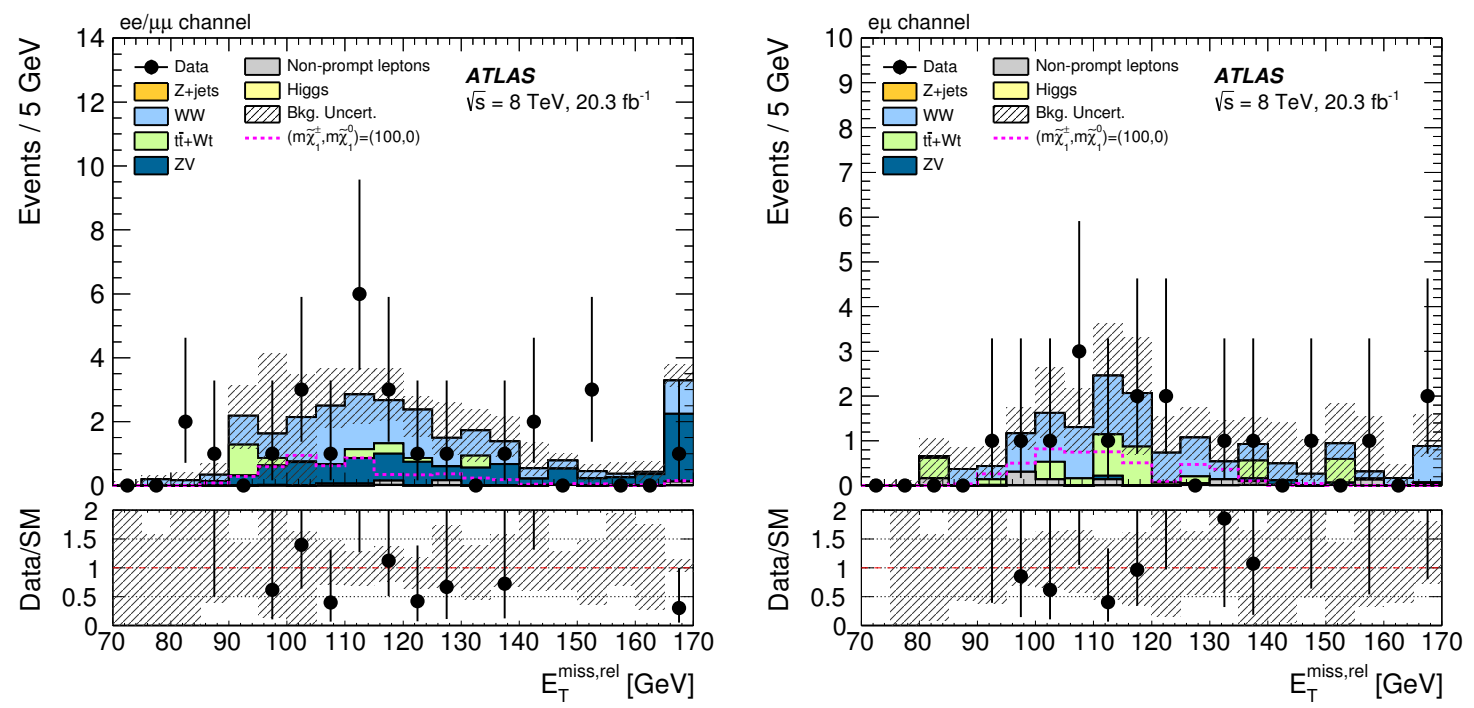

Figure 6.9: Kinematic distributions of $E_{\mathrm{T}}^{\mathrm{miss}, \mathrm{rel}}$ in the $S R-W W b$ region for the $S F$ channel (left) and the DF channel (right). An example signal point corresponding to the Simplified Model $\tilde{\chi}_{1}^{ \pm} \tilde{\chi}_{1}^{ \pm} \rightarrow W W$ with $\left(m_{\tilde{\chi}_{1}^{ \pm}}, m_{\tilde{\chi}_{1}^{0}}\right)=(100,0)$ GeV is overlaid.
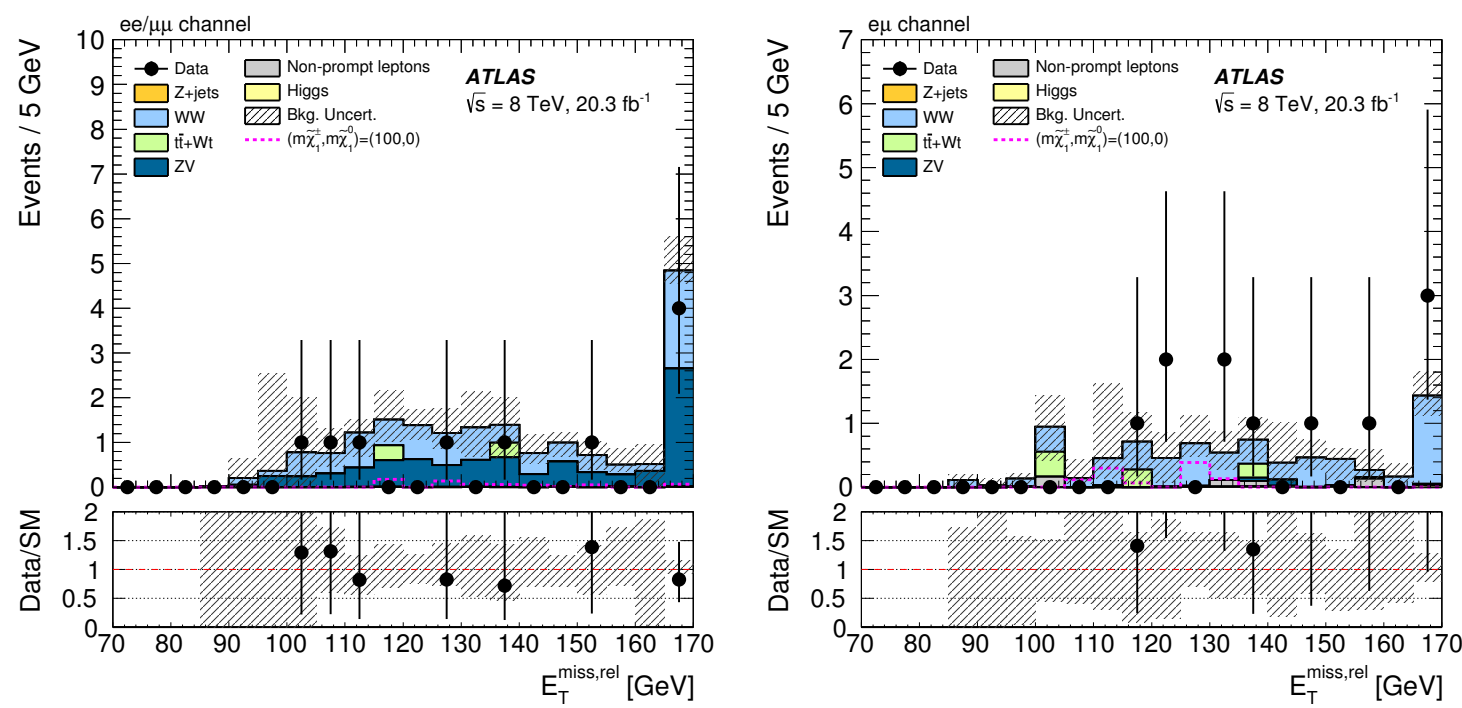

Figure 6.10: Kinematic distributions of $E_{\mathrm{T}}^{\mathrm{miss}, \mathrm{rel}}$ in the $S R$-WW c region for the $S F$ channel (left) and the DF channel (right). An example signal point corresponding to the Simplified Model $\tilde{\chi}_{1}^{ \pm} \tilde{\chi}_{1}^{ \pm} \rightarrow W W$ with $\left(m_{\tilde{\chi}_{1}^{ \pm}}, m_{\tilde{\chi}_{1}^{0}}\right)=(100,0)$ GeV is overlaid. 


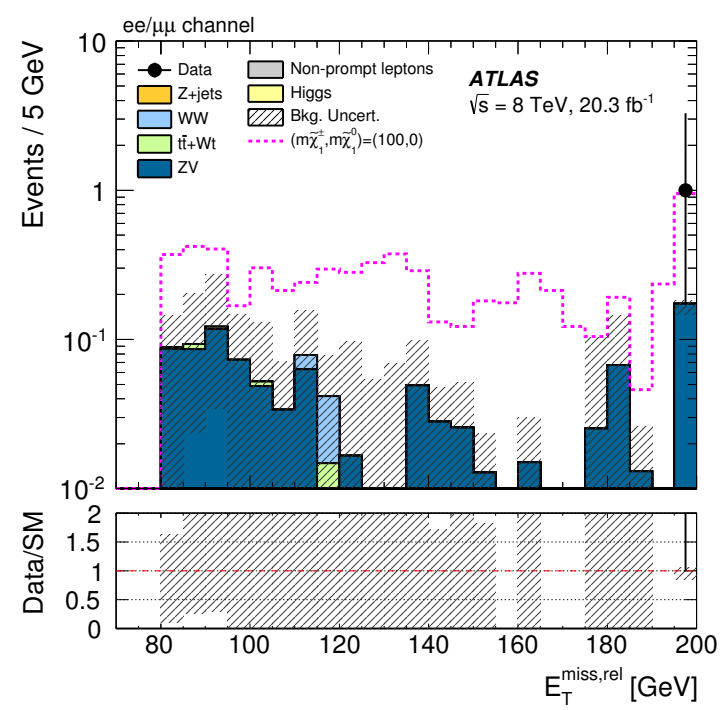

Figure 6.11: Kinematic distribution of $E_{\mathrm{T}}^{\mathrm{miss}, \mathrm{rel}}$ in the SR-Zjets region for the $S F$ channel. An example signal point corresponding to the Simplified Model $\tilde{\chi}_{1}^{ \pm} \tilde{\chi}_{2}^{0} \rightarrow Z W$ with $\left(m_{\tilde{\chi}_{1}^{ \pm}}, m_{\tilde{\chi}_{2}^{0}}\right)=(100,0)$ GeV is overlaid.

prediction without the SUSY signal.

\subsubsection{Kinematic Distributions in SR-Zjets}

Figure 6.11 shows the distribution of the $E_{\mathrm{T}}^{\text {miss,rel }}$ variable in the SR-Zjets region (summarized in Table 5.1). Since SR-Zjets is only defined in the SF channel, only one distribution is presented. For this SR, an example signal point of the Simplified Model $\tilde{\chi}_{1}^{ \pm} \tilde{\chi}_{2}^{0} \rightarrow Z W$ with $\left(m_{\tilde{\chi}_{1}^{ \pm}}, m_{\tilde{\chi}_{2}^{0}}\right)=(100,0) \mathrm{GeV}$ is overlaid.

Due to the strict selection criteria, only one observed event survives the selection. By noting the difference between the SM background (filled histogram) and the SUSY signal (dotted histogram), it can be preliminarily concluded that the data seem to follow the case without the SUSY signal. 


\subsubsection{Event Visualization of Candidate Events}

In this section, visualizations of an example candidate event in each of the SR are presented, which depict the particle trajectories within the ATLAS detector. These visualizations help to show that the methodology followed in this thesis selects wellidentified objects that are clean and isolated, as well as to show the clear imbalance of transverse momentum that is one of the predicted signatures of an EW SUSY event. The visualization is created using VP1 software [85] using the Athena framework version 17.2.8.13.

Figures $6.12,6.13$, and 6.14 are example candidate events in the $e e, \mu \mu$, and $e \mu$ channels, selected by the SR- $m_{\mathrm{T} 2} \mathrm{a}$, SR-Zjets, and SR- $W W$ a, respectively. For each visualization, the large yellow towers represent the electrons depositing energy in the calorimeter cells, the orange lines represent the muons which were detected by the muon chambers (represented by the green and the purple blocks), and the red dotted lines represent the direction of the missing momentum in the transverse plane. 


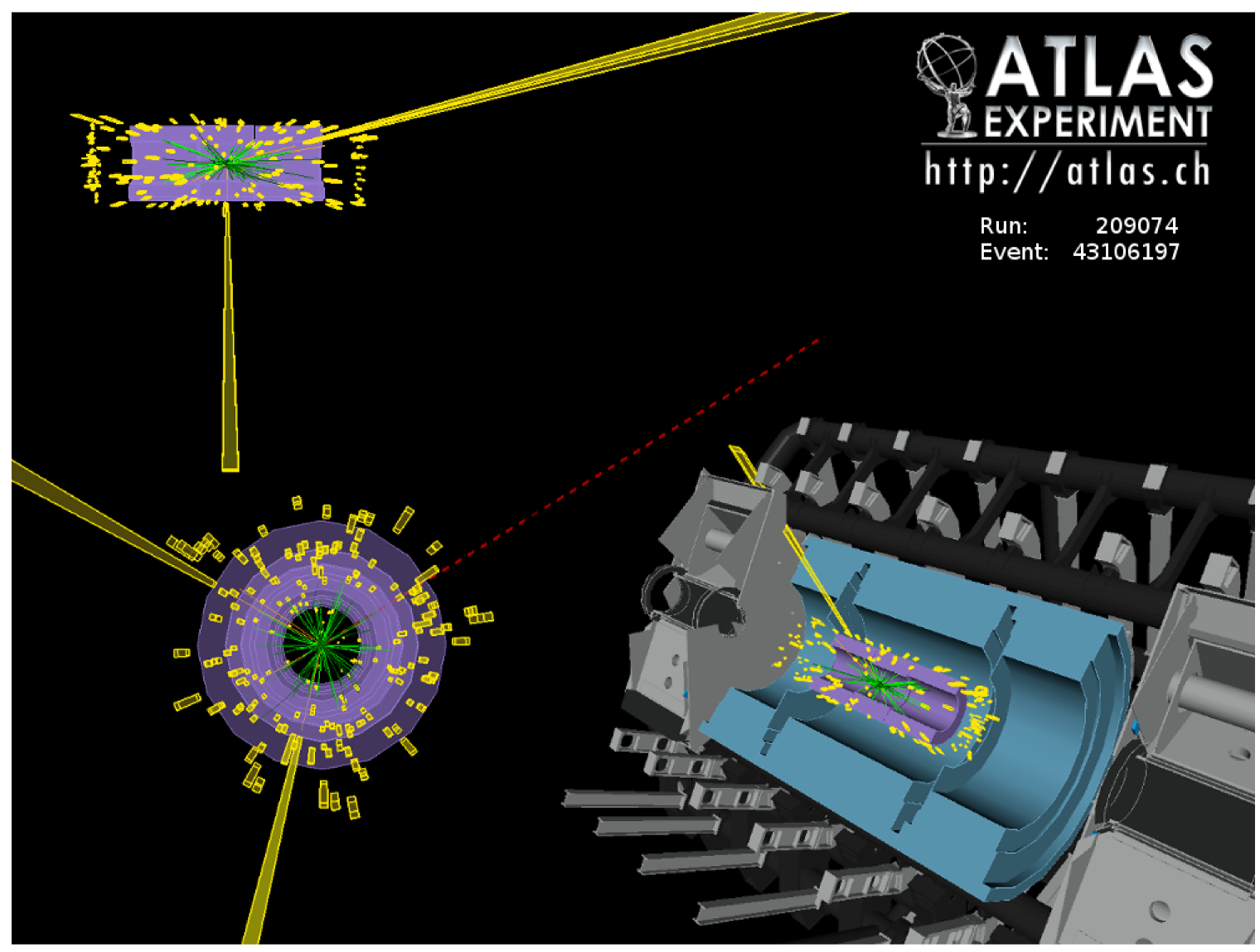

Figure 6.12: Visualization of an event in the ee channel. This event corresponds to Run \# 209074 and selected by $S R-m_{\mathrm{T} 2} a$. The cross sectional view in the transverse plane (side view) are drawn on the bottom (top) left of the picture, while the 3-D visualization is represented on the bottom right. 


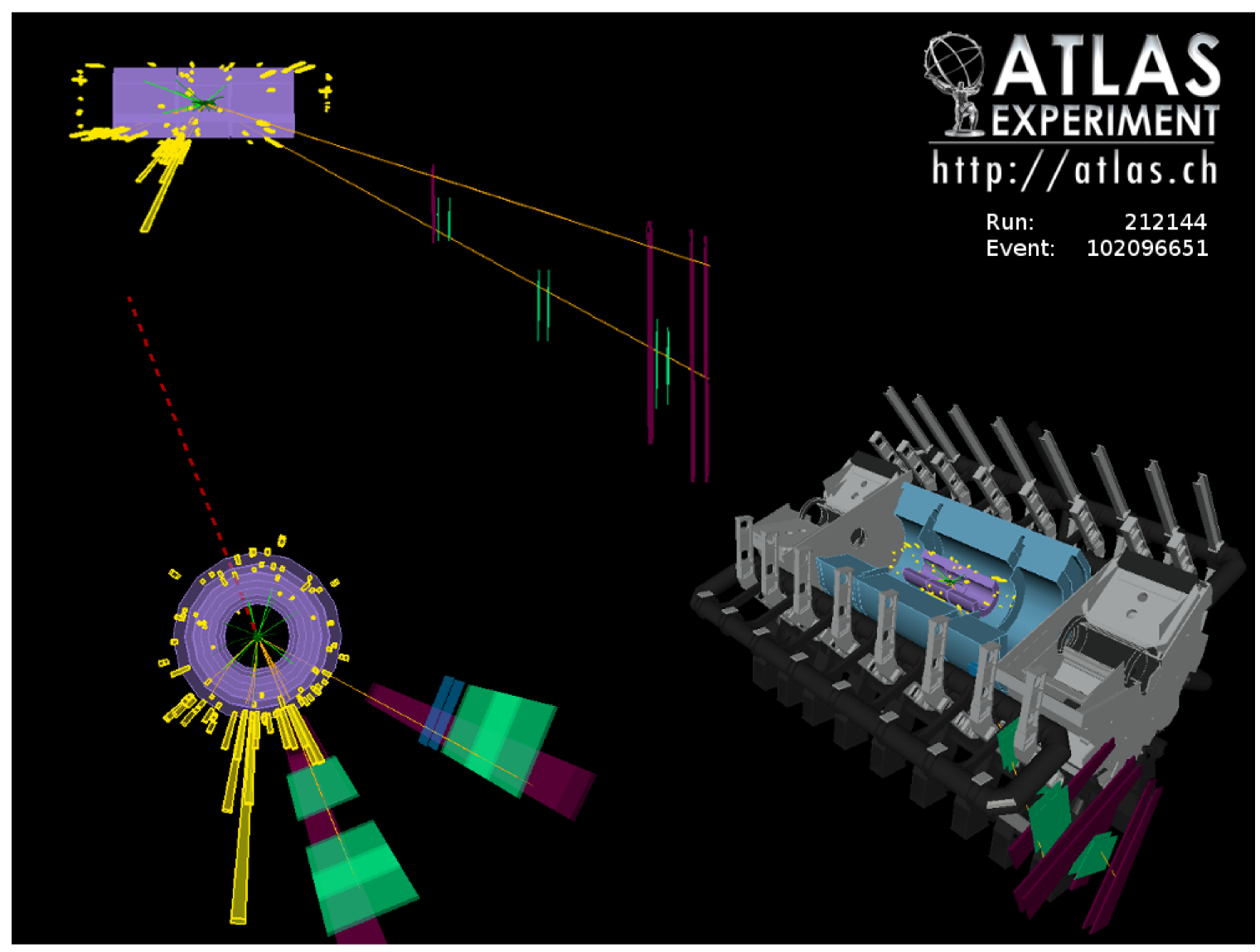

Figure 6.13: Visualization of an event in the $\mu \mu$ channel. This event corresponds to Run \# 212144 and selected by SR-Zjets. The cross sectional view in the transverse plane (side view) are drawn on the bottom (top) left of the picture, while the 3-D visualization is represented on the bottom right. 


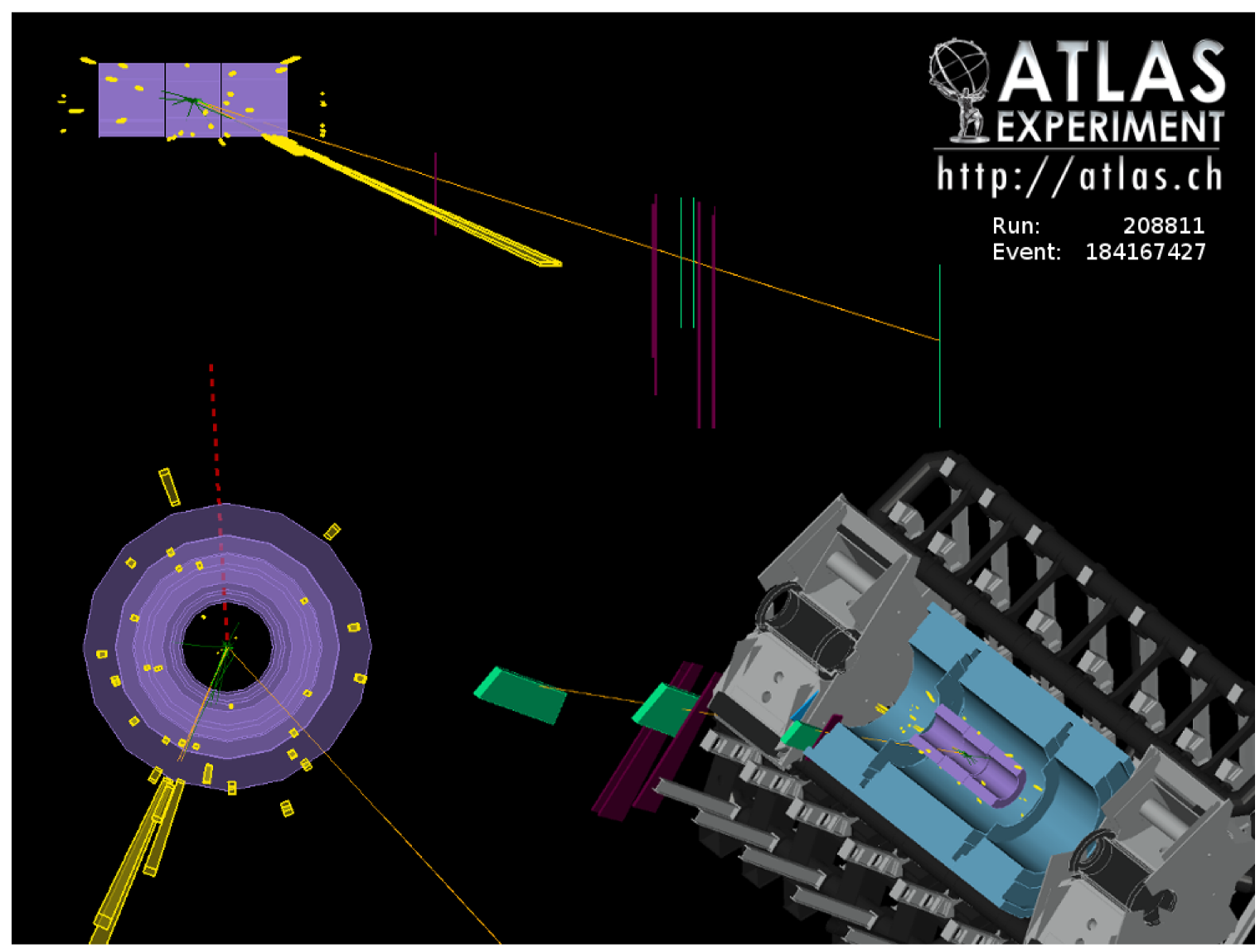

Figure 6.14: Visualization of an event in the e $\mu$ channel. This event corresponds to Run \# 208811 and selected by $S R-W W$ a. The cross sectional view in the transverse plane (side view) are drawn on the bottom (top) left of the picture, while the 3-D visualization is represented on the bottom right. 


\subsection{Background-Only Fit}

The purpose of the background-only fit is to establish the null hypothesis (i.e. absence of the signal events). This is done by performing a fit of the dominant SM background to the data in the control regions (CR) using the profile likelihood ratio (Equation 6.5), and determining the normalization of these backgrounds which are the free parameters to the fit (the background normalization factors $\mu_{b}$ in Equation 6.4). In practice, this normalization corrects for the mismodelling of MC samples compared to data. The fit is performed simultaneously on all CR defined for each of the dominant background components.

\subsubsection{Fit Results in the Control Regions}

Table 6.1 shows the result of the fit in the CR associated to the SR- $m_{\mathrm{T} 2}$ signal region in the DF channel for illustrative purposes. In the table, the "MC exp" corresponds to the raw event counts from the MC samples, the "fitted" corresponds to the event counts after undergoing the simultaneous fitting procedure, and "observed" is the raw data counts. As it can be seen, each CR is dominated by one background component of interest, and the fitted yields agree well with the observed data in each of the CR. There is no surviving $Z X$ event in these $\mathrm{CR}$, mainly due to the requirement of $\mathrm{DF}$ leptons. Figure 6.15 shows the effect of the fit, where the discrepancy in data and $\mathrm{MC}$ is visible before the fit and corrected after the fit. This is most visible in the $W W \mathrm{CR}$, where the $W W$ is scaled up by $\sim 14 \%$ (which is consistent with the scale factor quoted in Reference [76] and used for optimization study in Section 5.2).

The normalization factors are essentially the ratio of fitted events to the expected 


\begin{tabular}{lrrr}
\hline channel & Top CR & WW CR & ZV CR \\
\hline Observed events & 804 & 1061 & 94 \\
\hline Fitted bkg. events & $804.10 \pm 28.36$ & $1060.98 \pm 32.81$ & $94.01 \pm 9.77$ \\
\hline Fitted top events & $792.33 \pm 28.45$ & $110.43 \pm 19.20$ & $0.02_{-0.02}^{+2.41}$ \\
Fitted WW events & $11.18 \pm 1.57$ & $898.04 \pm 40.52$ & $4.73 \pm 0.50$ \\
Fitted ZV events & $0.43 \pm 0.17$ & $20.75 \pm 2.55$ & $89.19 \pm 9.79$ \\
Fitted ZX events & $0.00 \pm 0.00$ & $0.00 \pm 0.00$ & $0.00 \pm 0.00$ \\
Fitted fakes events & $0.00 \pm 0.00$ & $19.72 \pm 10.69$ & $0.00 \pm 0.00$ \\
Fitted higgs events & $0.15 \pm 0.06$ & $12.03 \pm 1.67$ & $0.07 \pm 0.03$ \\
\hline MC exp. SM events & 789.03 & 946.98 & 90.70 \\
\hline MC exp. top events & 778.67 & 108.51 & 0.65 \\
MC exp. WW events & 9.79 & 786.70 & 4.14 \\
MC exp. ZV events & 0.42 & 19.97 & 85.83 \\
MC exp. ZX events & 0.00 & 0.00 & 0.00 \\
MC exp. fakes events & 0.00 & 19.76 & 0.00 \\
MC exp. higgs events & 0.15 & 12.03 & 0.07 \\
\hline
\end{tabular}

Table 6.1: Background fit results for the top $C R, W W C R$, and $Z V$ CR for $S R-m_{\mathrm{T} 2}$ in the DF channel, for an integrated luminosity of $20.3 \mathrm{fb}^{-1}$. The errors shown are statistical plus systematic uncertainties.

\begin{tabular}{|c|c|c|}
\hline Top $\left(\mu_{b}^{\text {top }}\right)$ & $W W\left(\mu_{b}^{W W}\right)$ & $Z V\left(\mu_{b}^{Z V}\right)$ \\
\hline $1.017 \pm 0.0424$ & $1.138 \pm 0.0514$ & $1.028 \pm 0.1127$ \\
\hline
\end{tabular}

Table 6.2: Fitted background normalization factors for top $C R, W W C R$, and $Z V$ $C R$ for $S R-m_{\mathrm{T} 2}$ in the DF channel. These normalization factors are carried over to the $S R$.

MC events, evaluated for each CR simultaneously (3 parameter fit). Table 6.2 summarizes the normalization factors obtained from the fit. These normalization factors are then used to estimate the background compositions in the SR (in this case, SR- $\left.m_{\mathrm{T} 2}\right)$. 

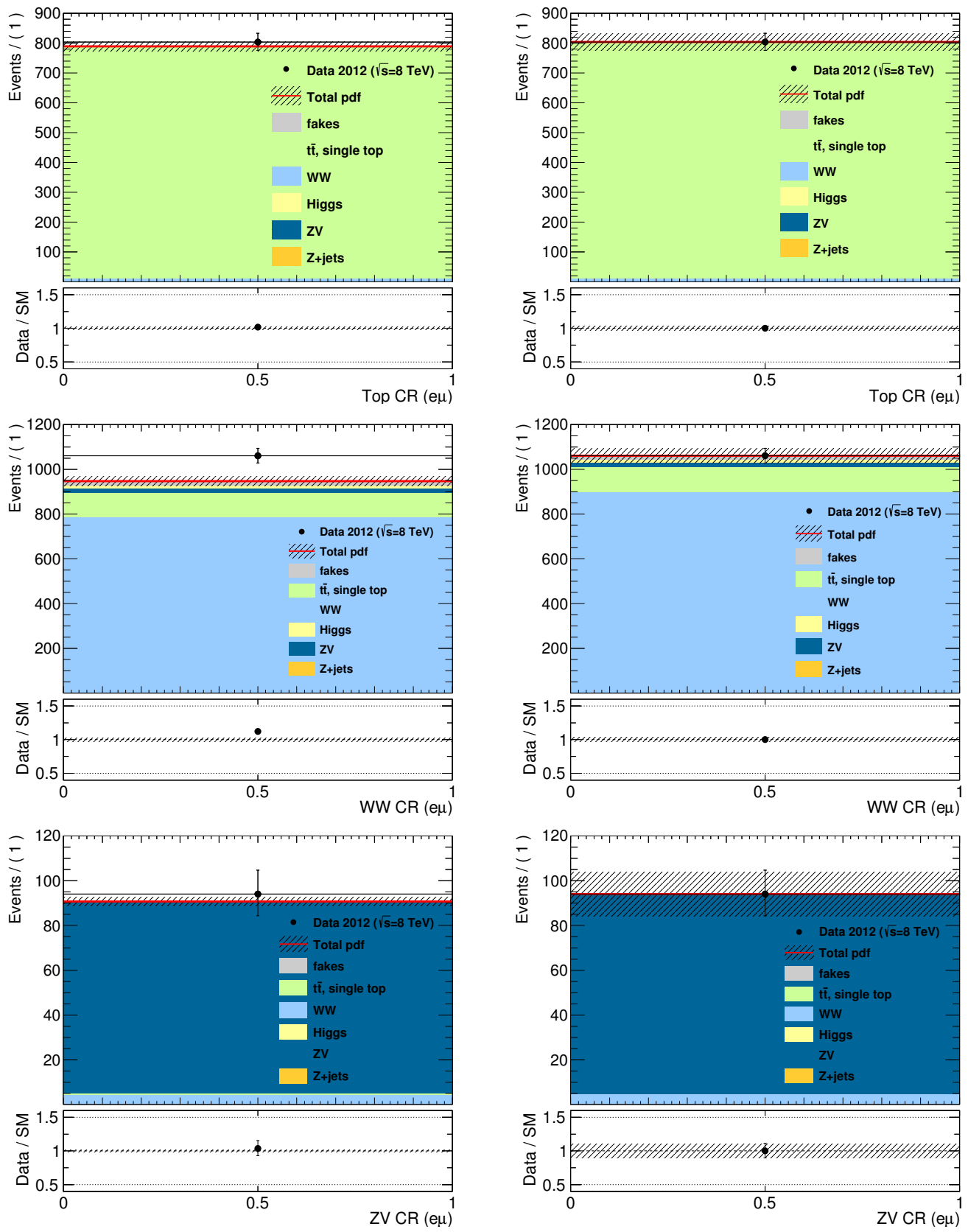

Figure 6.15: Stacked histograms of the SM background contributions before (left) and after (right) the simultaneous fit in the top CR (top), WW CR (middle) and $Z V$ $C R$ (bottom) for $S R-m_{\mathrm{T} 2}$. 


\begin{tabular}{|c|c|c|c|c|c|c|}
\hline & \multicolumn{2}{|c|}{ SR-mT2a } & \multicolumn{2}{|c|}{ SR-mT2b } & \multicolumn{2}{|c|}{ SR-mT2c } \\
\hline & $\mathrm{SF}$ & DF & $\mathrm{SF}$ & DF & $\mathrm{SF}$ & DF \\
\hline Top & $3.04 \pm 1.74$ & $5.53 \pm 1.87$ & $0.33_{-0.33}^{+0.36}$ & $0.00 \pm 0.00$ & $0.00 \pm 0.00$ & $0.00 \pm 0.00$ \\
\hline WW & $22.05 \pm 4.26$ & $16.18 \pm 3.21$ & $3.49 \pm 1.29$ & $3.29 \pm 1.19$ & $0.98 \pm 0.53$ & $0.90 \pm 0.46$ \\
\hline ZV & $12.85 \pm 2.23$ & $0.76 \pm 0.22$ & $4.94 \pm 1.62$ & $0.15 \pm 0.08$ & $2.15 \pm 0.49$ & $0.03 \pm 0.02$ \\
\hline ZX & $0.00_{-0.00}^{+0.01}$ & $0.00 \pm 0.00$ & $0.00 \pm 0.00$ & $0.00 \pm 0.00$ & $0.00 \pm 0.00$ & $0.00 \pm 0.00$ \\
\hline Fakes & $0.00 \pm 0.00$ & $0.59 \pm 0.55$ & $0.00 \pm 0.00$ & $0.01_{-0.01}^{+0.09}$ & $0.05_{-0.05}^{+0.12}$ & $0.00 \pm 0.00$ \\
\hline Higgs & $0.18 \pm 0.06$ & $0.19 \pm 0.05$ & $0.09 \pm 0.03$ & $0.10 \pm 0.04$ & $0.02 \pm 0.01$ & $0.04 \pm 0.03$ \\
\hline Total Bkg & $\overline{38} . \overline{1} \overline{ \pm} \overline{5} . \overline{0} 8$ & $2 \overline{3} . \overline{2} \pm 3.7 \overline{1}$ & $8 . \overline{8} \overline{\mathbf{E}} \overline{2} . \overline{0} \mathrm{8}$ & $3.5 \overline{5} \pm \overline{1} . \overline{2} 0$ & $3 . \overline{19} \pm 0 . \overline{7} 2$ & $\overline{0} . \overline{9} \pm 0 . \overline{4}$ \\
\hline Observed & 33 & 21 & 5 & 5 & 3 & 2 \\
\hline
\end{tabular}

Table 6.3: Result of the fitted background yield and the observed data count in SR$m_{\mathrm{T} 2}$ for SF and DF channels. The total systematic and statistical uncertainties are quoted.

\subsubsection{Fit Results in the Signal Regions}

The normalization factors obtained from the fit result in the CR are applied to each of the SR to identify the background composition in the SR. Figure 6.16 shows the fitted agreement of the data and the $\mathrm{MC}$ in the $\mathrm{CR}$ (top, WW, and ZV backgrounds are fitted simultaneously in their respective CR and correspond to the stacked histograms in Figure 6.15) as well as for the $\mathrm{SR}$ in the $\mathrm{SR}-m_{\mathrm{T} 2}$ regions. In the $\mathrm{SR}$, the pull plots are also shown. The pull, defined as $\left(n_{o b s}-n_{\text {exp }}\right) / \sigma_{\text {tot }}$, where $n_{o b s}$ and $n_{\text {exp }}$ are the observed and expected yields in the $\mathrm{SR}$, and $\sigma_{\text {tot }}$ is the total uncertainty, depicts the overall correction of the MC expectation in the SR due to the fitting done in the CR.

Table 6.3 shows the fitted background composition with the total systematic and statistical uncertainties (see Section 6.3.3 for the break-down), along with the observed data counts in the SR-mT2 signal regions. Overall good agreement between the fitted MC estimation and the observed data can be seen, suggesting no SUSY signal has been observed. 

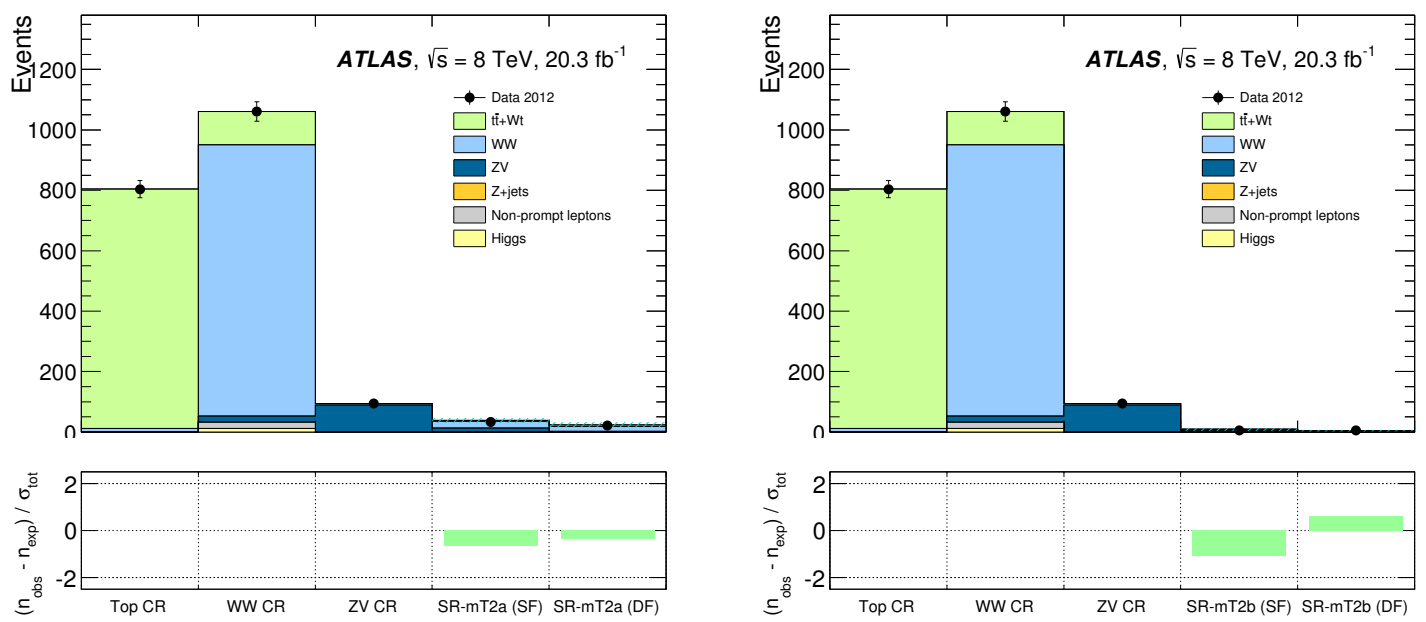

(a)

(b)

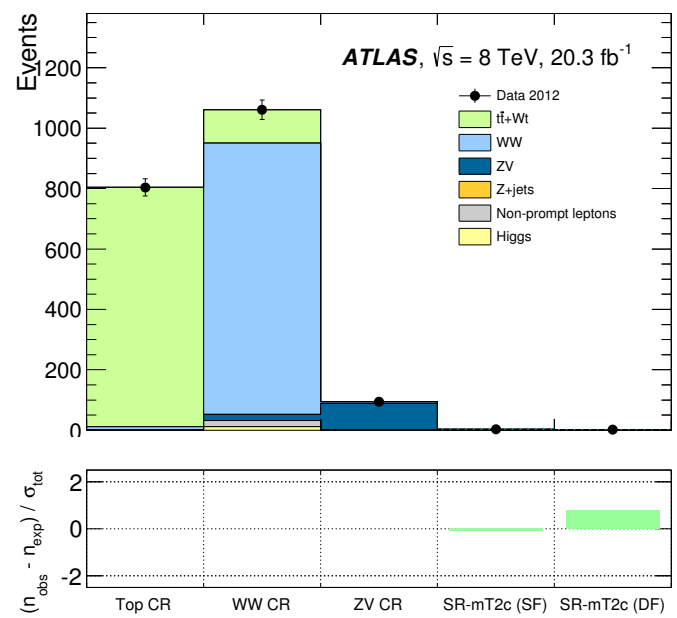

(c)

Figure 6.16: The fitted agreement in the top, $W W$, and $Z V C R$ and in $S R$ $m_{\mathrm{T} 2} a(a), S R-m_{\mathrm{T} 2} b(b), S R-m_{\mathrm{T} 2} c$ (c). The error bars on the data points are the statistical uncertainty, while the hashed region is the total uncertainty on the background estimate. The pull plot for the $S R$ is shown in the bottom pad.

Figure 6.17 shows the fitted agreement of the data and the MC in the $\mathrm{CR}$ as well as for the $\mathrm{SR}$ in the $\mathrm{SR}-W W$ regions. Note that the top, WW and ZV backgrounds 


\begin{tabular}{|c|c|c|c|c|c|c|}
\hline & \multicolumn{2}{|c|}{ SR-WWa } & \multicolumn{2}{|c|}{ SR-WWb } & \multicolumn{2}{|c|}{ SR-WWc } \\
\hline & $\mathrm{SF}$ & DF & $\mathrm{SF}$ & DF & $\mathrm{SF}$ & DF \\
\hline Top & $9.17 \pm 3.51$ & $11.55 \pm 4.30$ & $2.37 \pm 1.67$ & $4.31 \pm 1.60$ & $0.62_{-062}^{+1.15}$ & $0.94 \pm 0.83$ \\
\hline WW & $57.75 \pm 5.45$ & $58.21 \pm 5.99$ & $16.44 \pm 2.53$ & $12.30 \pm 2.00$ & $10.38 \pm 2.65$ & $7.29 \pm 1.93$ \\
\hline $\mathrm{ZV}$ & $16.31 \pm 3.50$ & $1.82 \pm 0.48$ & $10.91 \pm 1.91$ & $0.56 \pm 0.18$ & $9.21 \pm 2.12$ & $0.41 \pm 0.16$ \\
\hline ZX & $0.49_{-0.49}^{+0.68}$ & $0.00 \pm 0.00$ & $0.00_{-0.00}^{+0.01}$ & $0.00 \pm 0.00$ & $0.00 \pm 0.00$ & $0.00 \pm 0.00$ \\
\hline Non-prompt & $2.51 \pm 1.31$ & $1.72 \pm 1.10$ & $0.14_{-0.14}^{+0.31}$ & $0.72 \pm 0.55$ & $0.00 \pm 0.00$ & $0.26_{-0.26}^{+0.32}$ \\
\hline Higgs & $0.29 \pm 0.09$ & $0.29 \pm 0.07$ & $0.18 \pm 0.06$ & $0.19 \pm 0.05$ & $0.12 \pm 0.04$ & $0.14 \pm 0.04$ \\
\hline Total B $\bar{k}$ g & $\overline{8} 6 . \overline{5} \overline{ \pm} \overline{7} . \overline{3} 3$ & $\overline{73} . \overline{60} \pm \overline{7} . \overline{86}$ & $\overline{30} . \overline{0} 4 \pm 3.52$ & $\overline{18.07 \pm 2.59}$ & $\overline{2} 0 . \overline{34} \pm \overline{3} . \overline{5} \overline{4}$ & $\overline{9} . \overline{0} \pm 2 . \overline{9}$ \\
\hline Observed & 73 & 70 & 26 & 17 & 10 & 11 \\
\hline
\end{tabular}

Table 6.4: Result of the fitted background yield and the observed data counts in $S R-W W$ for SF and DF channels. The total systematic and statistical uncertainties are quoted.

are fitted simultaneously in this $\mathrm{SR}$, and $\mathrm{SR}-W W$ a has separate $\mathrm{CR}$ definition from $\mathrm{SR}-W W \mathrm{~b} / \mathrm{c}$ (which share the same $\mathrm{CR}$ as $\mathrm{SR}-m_{\mathrm{T} 2}$ ). In the $\mathrm{SR}$, pull plots are also shown.

Table 6.4 shows the fitted background composition with the total systematic and statistical uncertainties, along with the observed data counts in the SR- $W W$ signal regions. Once again, overall good agreement between the fitted MC estimation and the observed data can be seen (the biggest difference is seen for $\mathrm{SR}-W W \mathrm{c} \mathrm{SF}$ in Table 6.4, which is in agreement within three standard deviations).

Figure 6.18 shows the fitted agreement of the data and the $\mathrm{MC}$ in the $\mathrm{CR}$ (only the top CR is fitted in this SR) as well as for the SR in the SR-Zjets region. The pull plot is illustrated in the SR.

Finally, Table 6.5 shows the fitted background composition with the total systematic and statistical uncertainties, along with the observed data counts in the SR-Zjets signal region. Overall good agreement between the fitted $\mathrm{MC}$ estimation and the observed data can be seen. 


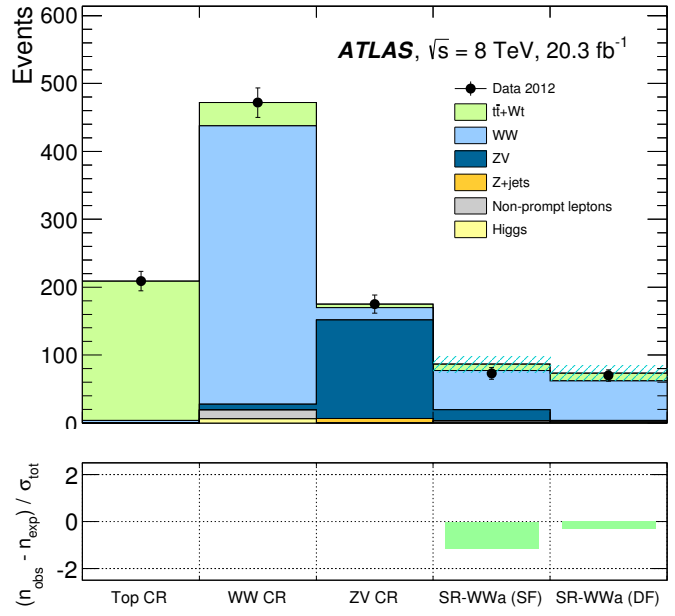

(a)

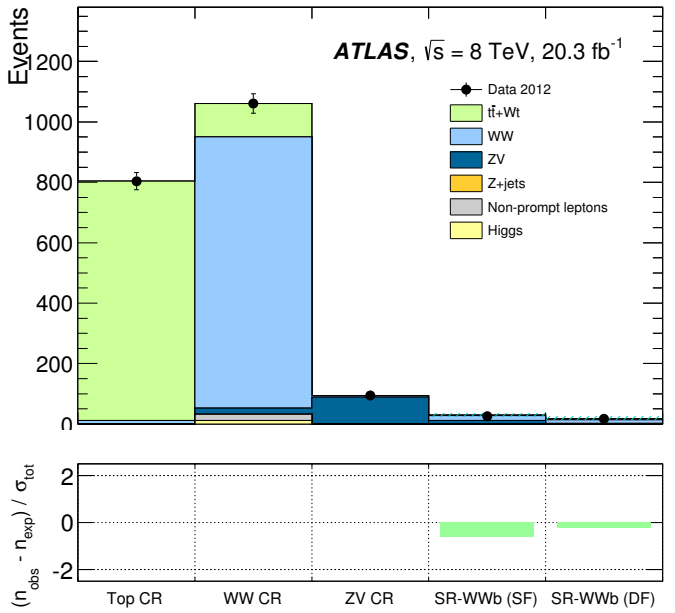

(b)
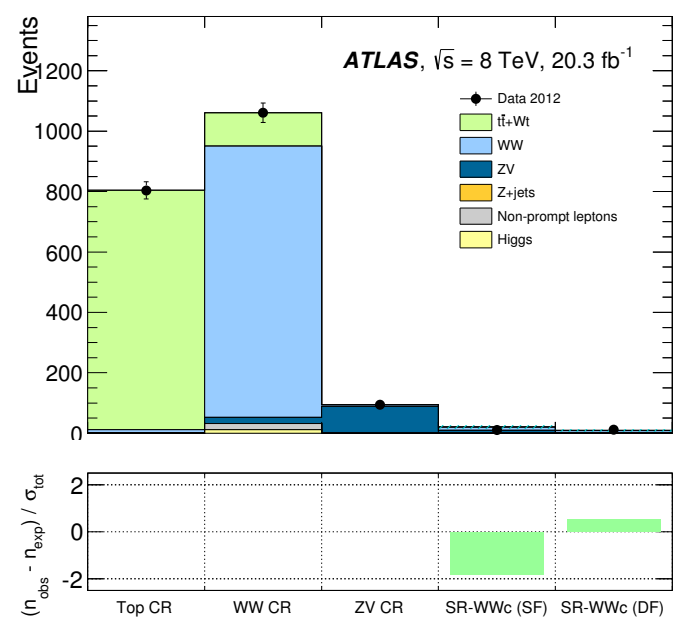

(c)

Figure 6.17: The fitted agreement in the top, $W W$, and $Z V C R$ and in $S R$ $W W a(a), S R-W W b(b), S R-W W c(c)$. The error bars on the data points are the statistical uncertainty, while the hashed region is the total uncertainty on the background estimate. The pull plot for the $S R$ is shown in the bottom pad. 


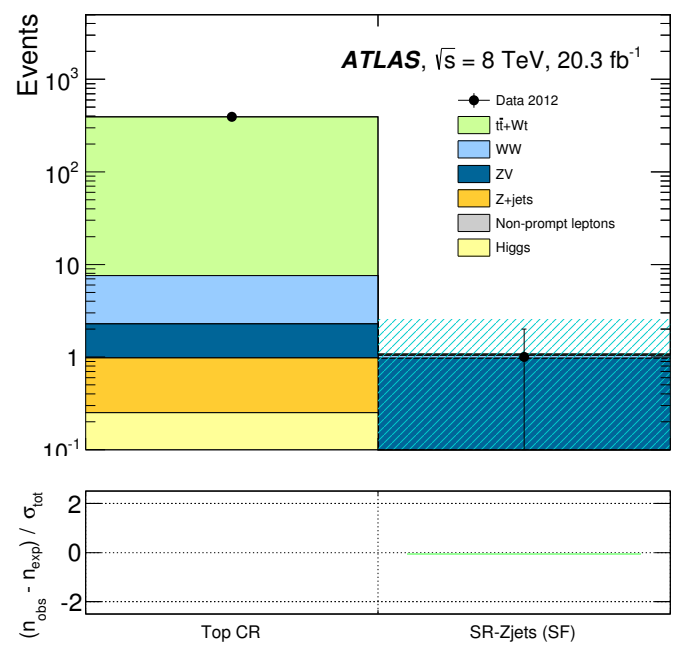

Figure 6.18: The fitted agreement in the top $C R$ and in SR-Zjets. The error bars on the data points are the statistical uncertainty, while the hashed region is the total uncertainty on the background estimate. The pull plot for the SR is shown in the bottom pad. Note the logarithmic scale on the vertical axis. Due to the lack of statistics (and hence the large statistical uncertainty), the pull is not visible.

\begin{tabular}{|l|c|}
\hline & $\begin{array}{c}\text { SR-Zjets } \\
\text { SF }\end{array}$ \\
\hline Top & $0.03 \pm 0.02$ \\
WW & $0.07_{-0.07}^{+0.09}$ \\
ZV & $0.98 \pm 0.62$ \\
ZX & $0.00 \pm 0.00$ \\
Non-prompt & $0.00 \pm 0.00$ \\
Higgs & $0.00 \pm 0.00$ \\
\hline$\overline{\text { Total }} \overline{\mathrm{Bkg}}-\overline{-}-\overline{1.07 \pm 0.6 \overline{3}}$ \\
\hline Observed & 1 \\
\hline
\end{tabular}

Table 6.5: Result of the fitted background yield and the observed data counts in SR-Zjets (only SF channel is applicable). The total systematic and statistical uncertainties are quoted. 


\begin{tabular}{l|c||c|c|c||c|c|c||c}
\hline & \multicolumn{1}{|c||}{ Chapter } & \multicolumn{3}{c||}{ SR- $m_{\mathrm{T} 2}$} & \multicolumn{3}{c||}{ SR- $W W$} & SR- $Z$ jets \\
& Section & $\mathrm{a}$ & $\mathrm{b}$ & $\mathrm{c}$ & $\mathrm{a}$ & $\mathrm{b}$ & $\mathrm{c}$ & \\
\hline Jet & 4.4 .1 & 3 & 2 & 6 & 5 & 3 & 4 & 11 \\
Lepton & 4.4 .2 & 2 & 1 & 3 & 2 & 3 & 5 & 4 \\
Missing Energy & 4.4 .3 & 3 & 1 & 5 & 1 & 4 & 4 & 5 \\
b-tagging & 4.4 .4 & 1 & $<1$ & $<1$ & 1 & 1 & 1 & 2 \\
Fake Lepton & 4.4 .5 & 1 & $<1$ & 1 & 1 & 1 & 1 & $<1$ \\
Modelling & 4.4 .6 & 12 & 26 & 29 & 6 & 9 & 17 & 42 \\
Luminosity & 4.4 .7 & $<1$ & $<1$ & $<1$ & $<1$ & $<1$ & $<1$ & 2 \\
MC Statistics & & 6 & 10 & 16 & 4 & 6 & 8 & 14 \\
\hline Total & & 15 & 29 & 30 & 10 & 13 & 21 & 47 \\
\hline
\end{tabular}

Table 6.6: Break-down of the evaluated values of the systematic uncertainties in the SR. The total values correspond to individual components added in quadrature. The second column from the left references the section where the respective systematic category is described. The quoted numbers are averages of the SF and the DF channels and are expressed as a percentage (\%) with respect to the nominal value of the total background estimate.

\subsubsection{Evaluation of the Systematic Uncertainties}

Descriptions of the systematic uncertainties are given in Section 4.4, and these uncertainties affect the estimate of the background and the signal yields. These also play a role in the hypothesis testing process as nuisance parameters.

Table 6.6 summarizes the break-down of the uncertainty values for each of the systematic categories as well as statistical uncertainties for each SR (for technical reasons, statistical uncertainty is a part of systematic uncertainties). The values are quoted as an average of the SF and the DF values and presented as a percentage value (\%) with respect to the nominal value of the total background estimate. Tables 6.36.5 in the previous section quoted the total uncertainties (including the statistical uncertainty) as given in Table 6.6. As it can be seen, the dominant systematic uncertainty for all SR is associated with the MC modelling of the backgrounds. 
The values quoted in the table can be reasonably explained. For instance, the MC statistical uncertainties increase with more stringent selection. The modelling uncertainty is expected to be large because the modelling of particle interactions is affected by several factors, such as the choices of event generation models, initialand final-state radiation models, and cross section models. The difference in event generation models used for the estimation of the $W W$ and the $Z V$ background contributes dominantly to the modelling uncertainty.

\subsection{Model-Independent Fit (Discovery Fit)}

After the null hypothesis is established with the background-only fitting procedure, the model-independent fit can be utilized to test the alternate hypothesis, that is, to calculate the discovery potential, $p_{\mu}$. The fit will test for the excess above the SM cross section without assuming the nature of the new physics, and this will establish an upper limit on the cross section for new physics beyond the SM. This is achieved by making the signal contribution in the SR a free-parameter in the fit $\left(\mu_{s}\right.$ in Equation 6.4). The background normalization factors $\mu_{b}$ are also refitted.

Table 6.7 shows the result of the fit for each SR. The values of $C L_{b}$ and $p_{\mu}$, as well as the number of signal counts, are obtained from the fit. The cross section values ( $\epsilon \sigma$, where $\epsilon$ is the efficiency factor for the SR requirements) are calculated by dividing the number of signal counts (equivalent to the signal strength parameter $\mu_{s}$ ) by the integrated luminosity for the 2012 data, $L=20.3 \mathrm{fb}^{-1}$. The $\epsilon$ factor signifies the cross section is the result of inspecting the signal counts in a SR, which is only a subset of the full dataset. In order for the alternative hypothesis to be favourable, a $p$-value of $\leq 5 \%$ is needed. As it can be seen from the table, the lowest $p$-value is 0.22 


\begin{tabular}{|c|c|c|c|c|c|c|}
\hline Signal channel & $\langle\epsilon \sigma\rangle_{\text {obs }}^{95}[\mathrm{fb}]$ & $\langle\epsilon \sigma\rangle_{\exp }^{95}[\mathrm{fb}]$ & $S_{\mathrm{obs}}^{95}$ & $S_{\exp }^{95}$ & $C L_{b}$ & $p_{\mu}$ \\
\hline SR-mT2a SF & 0.64 & $0.79_{-0.22}^{+0.32}$ & 13.0 & $16.1_{-4.6}^{+6.5}$ & 0.27 & 0.50 \\
\hline SR-mT2a DF & 0.56 & $0.63_{-0.18}^{+0.26}$ & 11.4 & $12.8_{-3.7}^{+5.3}$ & 0.37 & 0.50 \\
\hline$\overline{\mathrm{SR}}-\overline{\mathrm{m}} \overline{\mathrm{T}} \overline{2} \overline{\mathrm{b}} \overline{\mathrm{S}} \overline{\mathrm{F}}$ & $0 . \overline{2} 6$ & $0 . \overline{3} 7_{-0.11}^{+0.17}$ & $\overline{5} . \overline{3}^{-}$ & $7.5_{-2.2}^{+3.5}$ & $\overline{0} . \overline{1} \overline{6}$ & $0 . \overline{5} 0$ \\
\hline SR-mT2b DF & 0.35 & $0.29_{-0.08}^{+0.14}$ & 7.2 & $5.9_{-1.7}^{+2.9}$ & 0.69 & 0.30 \\
\hline$\overline{\mathrm{SR}}-\overline{\mathrm{m}} \overline{\mathrm{T}} \overline{2} \mathrm{c} \overline{\mathrm{S}} \overline{\mathrm{F}}-$ & $0 . \overline{2} \overline{4}$ & $0.25_{-0.08}^{+0.13}$ & $\overline{4} . \overline{9}$ & $5.1_{-1.7}^{+2.7}$ & $\overline{0 .} . \overline{4}$ & $0 . \overline{5} 0$ \\
\hline SR-mT2c DF & 0.24 & $0.18_{-0.06}^{+0.12}$ & 4.9 & $3.7_{-1.3}^{+2.3}$ & 0.72 & 0.22 \\
\hline$\overline{\text { SR-WWa SF }}$ & 0.80 & $1.15_{-0.32}^{+0.47}$ & 16.2 & $23.3_{-6.4}^{+9.5}$ & 0.13 & 0.50 \\
\hline SR-WWa DF & 1.02 & $1.13_{-0.31}^{+0.45}$ & 20.7 & $22.9_{-6.3}^{+9.2}$ & 0.38 & 0.50 \\
\hline$\overline{\mathrm{SR}} \overline{-\overline{\mathrm{W}}} \overline{\mathrm{W}} \overline{\mathrm{b}} \overline{\mathrm{S}} \overline{\mathrm{F}}$ & $0 . \overline{5} \overline{4}$ & $0 . \overline{6} 7_{-0.20}^{+0.29}$ & 11.0 & $\overline{1} \overline{3} . \overline{6}_{-4.0}^{+5.8}$ & $\overline{0} . \overline{2} \overline{8}$ & $0 . \overline{50}$ \\
\hline SR-WWb DF & 0.50 & $0.54_{-0.16}^{+0.23}$ & 10.2 & $10.9_{-3.2}^{+4.8}$ & 0.42 & 0.50 \\
\hline$\overline{\mathrm{SR}}-\overline{\mathrm{W}} \overline{\mathrm{W}} \bar{c}^{-} \overline{\mathrm{S}} \overline{\mathrm{F}}-$ & $\overline{0 . \overline{2}} \overline{-}$ & $0.52_{-0.16}^{+0.23}$ & $\overline{5} . \overline{9}^{-}$ & $\overline{10} . \overline{6}_{-3.2}^{\mp 4.6}$ & $\overline{0} . \overline{0} \overline{4}$ & $0 . \overline{50}$ \\
\hline SR-WWc DF & 0.50 & $0.42_{-0.12}^{+0.20}$ & 10.2 & $8.6_{-2.5}^{+3.9}$ & 0.67 & 0.31 \\
\hline$\overline{\text { SR-Zjets SF }}$ & 0.18 & $0.18_{-0.07}^{+0.11}$ & 3.6 & $3.6_{-1.3}^{+2.2}$ & 0.48 & 0.50 \\
\hline
\end{tabular}

Table 6.7: Left to right: 95\% CL upper limits on the observed (expected) cross section $\langle\epsilon \sigma\rangle_{\mathrm{obs}}^{95}\left(\langle\epsilon \sigma\rangle_{\mathrm{exp}}^{95}\right)$ and on the number of observed (expected) signal events $S_{\mathrm{obs}}^{95}$ $\left(S_{\exp }^{95}\right)$. The total systematic and statistical uncertainties are quoted for the expected values. The last two columns indicate the $C L_{b}$ value, i.e. the confidence level observed for the background-only hypothesis, and the discovery $p$-value $\left(p_{\mu}\right)$.

in SR- $m_{\mathrm{T} 2} \mathrm{c}$ (for technical reasons, the value of $p_{\mu}$ is capped at 0.50 ). This can be interpreted as having $22 \%$ chance that the alternate hypothesis is favoured as a result of random chance. This indicates that the SM describes the observation without the need for any new physics.

\subsection{Model-Dependent Fit (Exclusion Fit)}

The model-independent fit result did not favour the existence of SUSY (or any new physics beyond the SM). In such a case, a model-dependent fit is used to set the upper limit on parameters of a particular model using the $C L_{s}$ technique introduced 
in Section 6.1. For each signal grid point introduced in Table 4.3, the $C L_{s}$ value was calculated for each SR independently using the observed event yield and the expected signal count $s$ from the particular SR. Then, the $C L_{s}$ value corresponding to the SR with the best expected exclusion is used (for example, for $\left(\mu, M_{2}\right)=(100,100) \mathrm{GeV}$ point in Figure 6.19, SR- $W W$ a is used because $\left.\left.C L_{s}^{e x p}\right|_{\mathrm{SR}-\mathrm{WWa}}<\left.C L_{s}^{e x p}\right|_{\text {otherSR }}\right)$ to draw the contour line indicating $C L_{s}=0.05$.

In Section 3.4, specific SUSY models considered for this work were introduced. In this chapter, only the exclusion results for the pMSSM models are discussed, as they were my personal contributions to the analysis. The exclusion results for the Simplified Model can be found in Reference [28].

\subsection{1 pMSSM Direct Gaugino with Intermediate Sleptons}

The signal grid points based on the pMSSM Direct Gaugino (DG) models with intermediate sleptons have been parameterized by the gaugino mass parameters $\left(M_{1}\right.$, $M_{2}$, and $\mu$ ), while keeping the rest constant. A total of 448 grid points have been produced to scan through these mass parameters. $M_{1}$ values of $100 \mathrm{GeV}, 140 \mathrm{GeV}$ and $250 \mathrm{GeV}$ are tested. For each $M_{1}$ value, $M_{2}$ and $\mu$ are varied between $100 \mathrm{GeV}$ and $500 \mathrm{GeV}$ in steps ranging between $10 \mathrm{GeV}$ and $50 \mathrm{GeV}$, and the $C L_{s}$ values are calculated for each grid point.

Figures $6.19,6.20$ and 6.21 show the $95 \%$ exclusion contour for $M_{1}=100 \mathrm{GeV}$, $M_{1}=140 \mathrm{GeV}$, and $M_{1}=250 \mathrm{GeV}$ respectively. The expected exclusion contour is represented by the black dotted line, with the yellow band indicating $\pm 1 \sigma$ uncertainty. The pale green region indicates the expected excluded region, while the white region is not yet excluded. The observed exclusion contour is represented by the thick red line 

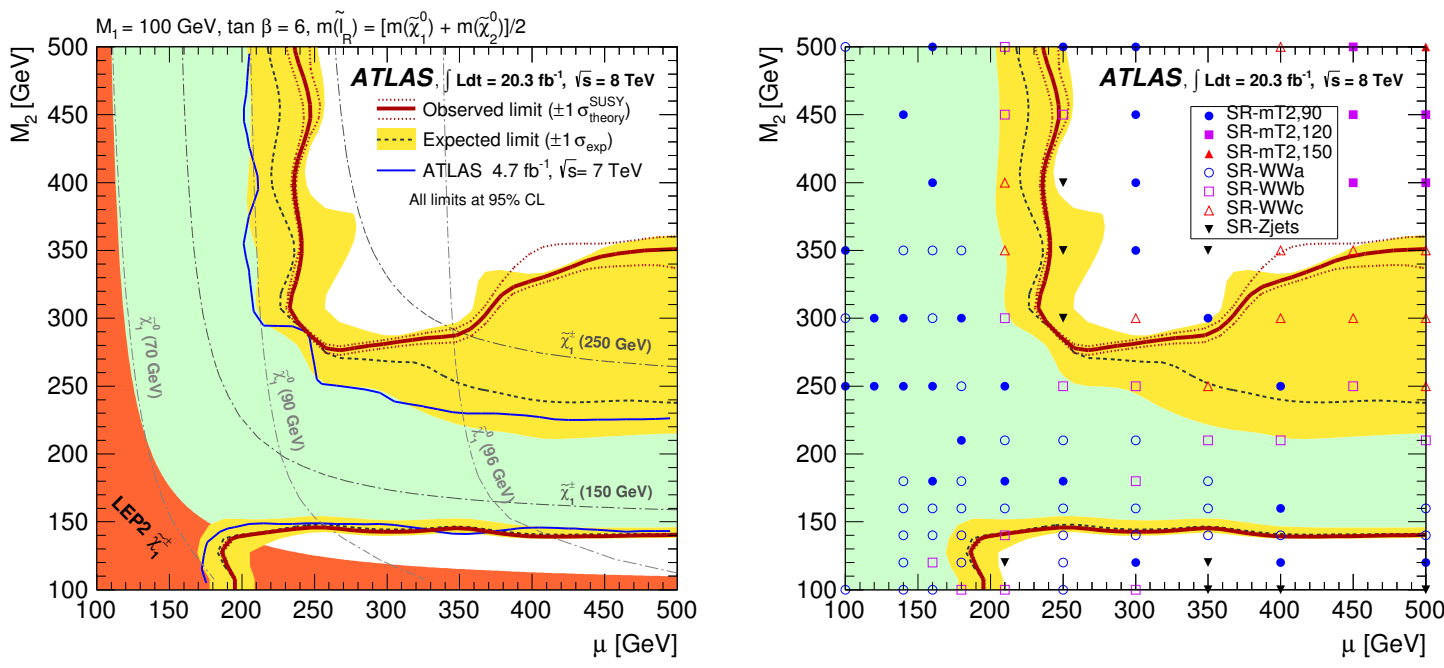

Figure 6.19: Exclusion plots for pMSSM DG with the gaugino mass parameter $M_{1}=100 \mathrm{GeV}$. The $\mu$ and $M_{2}$ gaugino mass parameters are varied along the $x$ and the $y$ axis, respectively. The black dotted line with yellow band represents the expected limit and the thick red line with the dotted red lines represents the observed limit. The pale green area represents the expected excluded region with $C L_{s}<0.05$ (extends up to the black dotted line). On the left, the LEP2 exclusion result, the ATLAS result from the $\sqrt{s}=7 \mathrm{TeV}$ analysis, and the masses of gauginos are overlaid. The right plot shows the mass configuration of each grid point, as well as the SR that resulted in the maximum expected $C L_{s}$ value.

with thin, dotted line indicating $\pm 1 \sigma$ uncertainty on the cross section for the model. For each figure, the right plot shows the map of the mass configuration for each signal sample. For each point, a marker indicates the SR used for the computation of the $C L_{s}$ value and for the contour. Aside from the contour lines, the previous upper limit from the LEP2 result [21] (orange filled contour) and from the $\sqrt{s}=7 \mathrm{TeV}$ analysis [86] (blue line) are overlaid, as well as the approximate $\tilde{\chi}_{1}^{0}$ and $\tilde{\chi}_{1}^{ \pm}$masses given the gaugino mass parameters.

For the $M_{1}=100 \mathrm{GeV}\left(M_{1}=140 \mathrm{GeV}\right)$ cases, the region within $M_{2}<150 \mathrm{GeV}$ $\left(M_{2}<200 \mathrm{GeV}\right)$ and $\mu>200 \mathrm{GeV}$ is not excluded. This can be explained by the fact that the $\tilde{\chi}_{1}^{ \pm}$and $\tilde{\chi}_{1}^{0}$ masses are close to each other (within $\sim 15 \mathrm{GeV}$ ) as it can be seen 

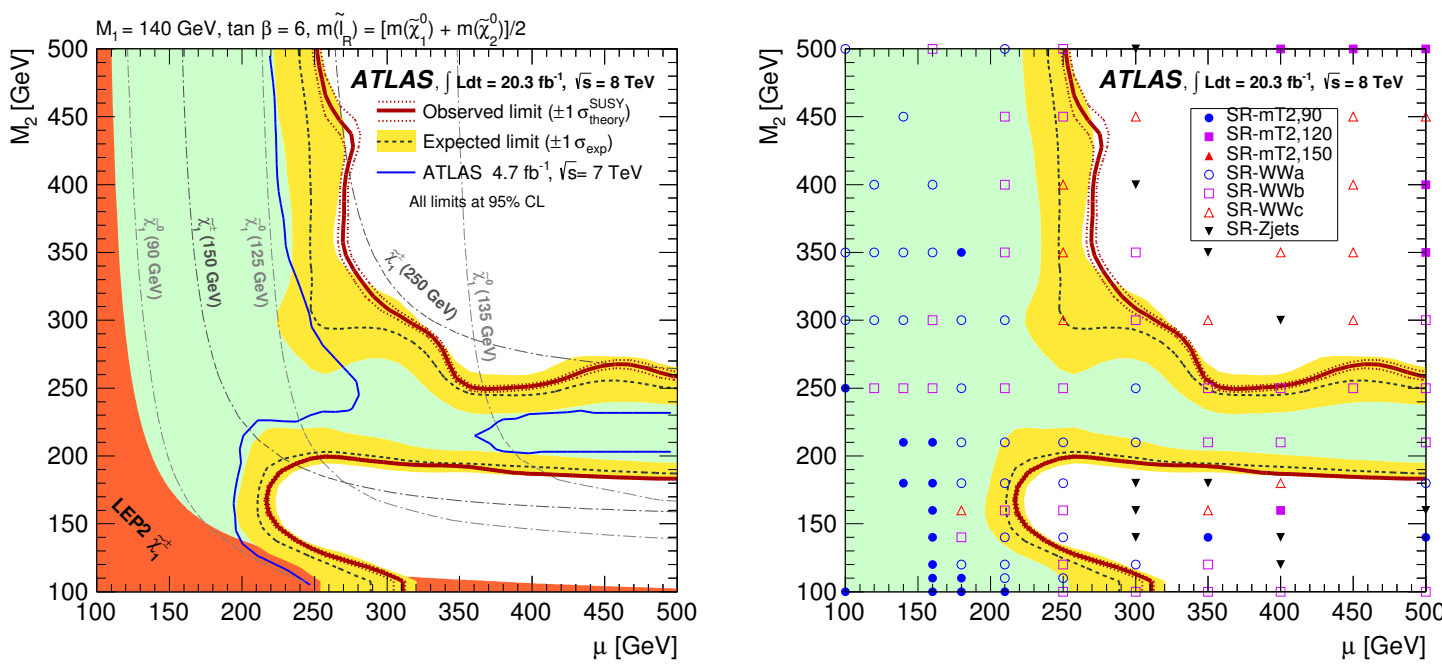

Figure 6.20: Exclusion plots for pMSSM DG with the gaugino mass parameter $M_{1}=140 \mathrm{GeV}$. The $\mu$ and $M_{2}$ gaugino mass parameters are varied along the $x$ and the $y$ axis, respectively. The black dotted line with yellow band represents the expected limit and the thick red line with the dotted red lines represents the observed limit. The pale green area represents the expected excluded region with $C L_{s}<0.05$ (extends up to the black dotted line). On the left, the LEP2 exclusion result, the ATLAS result from the $\sqrt{s}=7 \mathrm{TeV}$ analysis, and the masses of gauginos are overlaid. The right plot shows the mass configuration of each grid point, as well as the $S R$ that resulted in the maximum expected $C L_{s}$ value.

by the dashed mass lines in Figures 6.19(left) and 6.20(left). The signal topologies of interest (Figure 3.5) require that the final-state leptons originate from the decay of $\tilde{\chi}_{1}^{ \pm}$to $\tilde{\chi}_{1}^{0}$, but the small mass difference allows only low-energy leptons which may not pass the selection criteria in the $\mathrm{SR}$ (lepton $p_{\mathrm{T}}$ is required to be more than $20 \mathrm{GeV}$ in all SR, as summarized in Table 5.1). The high $M_{2}$ and $\mu$ region (top right) is not excluded mainly due to the low cross sections arising from higher $\tilde{\chi}^{ \pm}$and $\tilde{\chi}^{0}$ masses. It can be seen that for all three cases, the excluded region has been expanded since the $\sqrt{s}=7 \mathrm{TeV}$ result. 

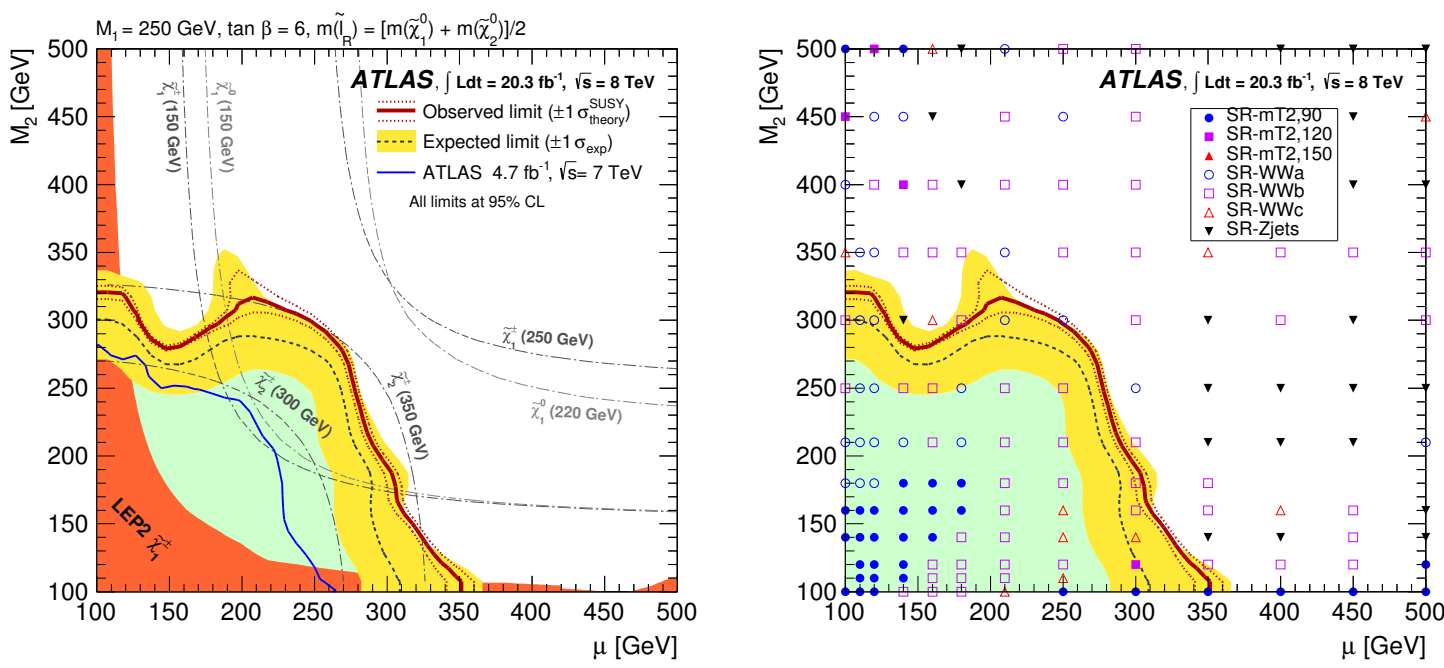

Figure 6.21: Exclusion plots for pMSSM DG with the gaugino mass parameter $M_{1}=250 \mathrm{GeV}$. The $\mu$ and $M_{2}$ gaugino mass parameters are varied along the $x$ and the $y$ axis, respectively. The black dotted line with yellow band represents the expected limit and the thick red line with the dotted red lines represents the observed limit. The pale green area represents the expected excluded region with $C L_{s}<0.05$ (extends up to the black dotted line). On the left, the LEP2 exclusion result, the ATLAS result from the $\sqrt{s}=7 \mathrm{TeV}$ analysis, and the masses of gauginos are overlaid. The right plot shows the mass configuration of each grid point, as well as the SR that resulted in the maximum expected $C L_{s}$ value.

\subsection{2 pMSSM Direct Gaugino without Intermediate Slep- tons}

As with pMSSM DG models with intermediate sleptons, the pMSSM DG models without the intermediate sleptons are also parameterized by the gaugino mass parameters $\left(M_{1}, M_{2}, \mu\right)$, and they are scanned through 99 grid points. For these models, $M_{1}$ is fixed at $50 \mathrm{GeV}$, while $M_{2}$ and $\mu$ are varied between $100 \mathrm{GeV}$ and $500 \mathrm{GeV}$ in steps of $50 \mathrm{GeV}$. The $C L_{s}$ values are calculated for each grid point, and the exclusion contours are drawn.

Figure 6.22 shows the $95 \%$ exclusion contour. As it can be seen, the SR-Zjets 

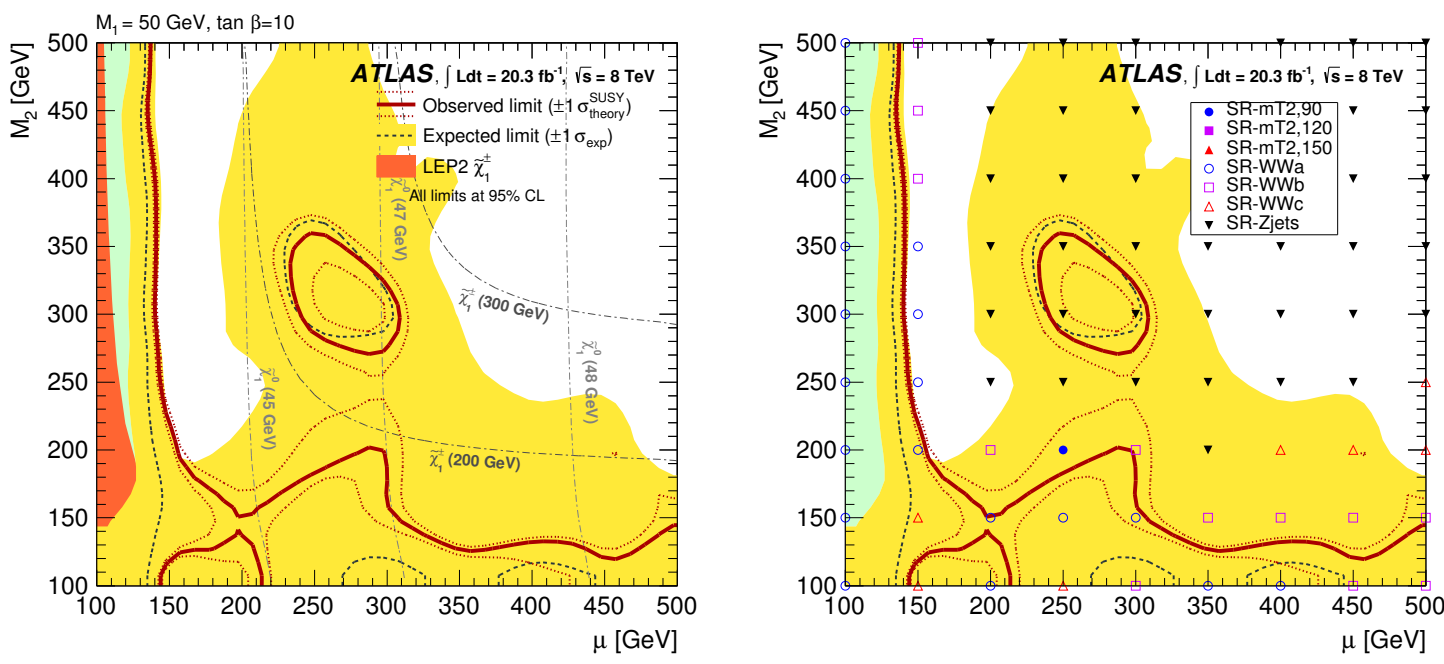

Figure 6.22: Exclusion plots for pMSSM DG without intermediate sleptons. The $\mu$ and $M_{2}$ gaugino mass parameters are varied along the $x$ and the $y$ axis, respectively, while $M_{1}$ is fixed at $50 \mathrm{GeV}$. The black dotted line with yellow band represents the expected limit and the thick red line with the dotted red lines represents the observed limit. The pale green area represents the expected excluded region. On the left, the LEP2 exclusion result and the masses of the gauginos are overlaid. The right plot shows the mass configuration of each grid point, as well as the SR with the best expected exclusion limit.

sensitivity is maximized at different region in the $M_{2}-\mu$ plane than the rest of the SR, and this results in an "island" of excluded region in the middle. SR-Zjets has good sensitivity in this region, because the $\tilde{\chi}_{1}^{ \pm}$and $\tilde{\chi}_{2}^{0}$ masses must be high enough to produce the intermediate $W$ and $Z$ bosons as illustrated in Figure 3.5d. A "hole" of non-excluded region at $\left(M_{2}, \mu\right)=(100,150-200) \mathrm{GeV}$ is due to a statistical fluctuation arising from limited signal yield with large MC correction factors. The pMSSM DG models without the intermediate sleptons were not studied with the $\sqrt{s}=7 \mathrm{TeV}$ data, therefore the result presented here is the first exclusion for these models. 

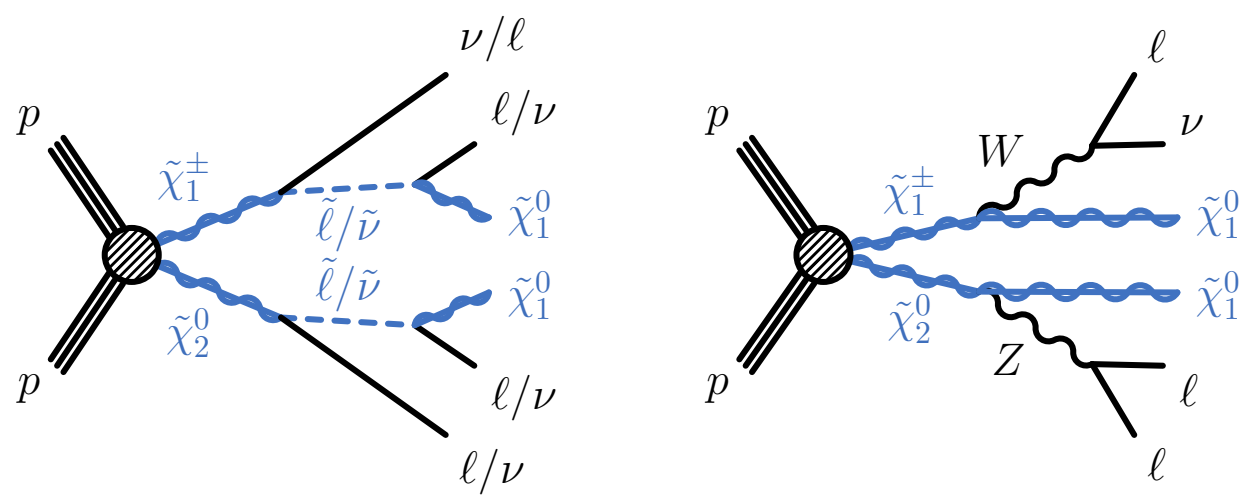

Figure 6.23: Signal topologies for Electroweak SUSY production with 3 finalstate leptons. $\tilde{\chi}_{1}^{ \pm} \tilde{\chi}_{2}^{0} \rightarrow 3 \ell$ with intermediate sleptons (left) and $\tilde{\chi}_{1}^{ \pm} \tilde{\chi}_{2}^{0} \rightarrow 3 \ell$ with intermediate $W / Z$ bosons (right).

\subsection{Combination with Trilepton Analysis}

This analysis considered the final-state lepton multiplicity to be exactly two. The EW SUSY production could form topologies that involve trilepton signature (3 final-state leptons) with very similar observables. Some examples of trilepton topologies are shown in Figure 6.23. Hence, it is expected to be sensitive to the same SUSY models as the dilepton analysis, namely the Simplified Model and the pMSSM models.

The results of exclusion limits for the dilepton final states are combined with the trilepton results in order to improve the upper limit on the gaugino mass parameters for the signal models under question. For this thesis, only the results from the pMSSM DG models are presented since this was my personal contribution. The exclusion plots for the Simplified Model can be found in Reference [28].

The combination process relies on statistically combining the $C L_{s}$ values of the dilepton and trilepton results $\left(C L_{s}^{c o m b}=C L_{s}^{2 L} \oplus C L_{s}^{3 L}\right)$ for each grid point. This 
procedure is valid since the SR used by the trilepton analysis have no overlaps with those used by the dilepton analysis.

Figures 6.24 and 6.25 show the results of the combination with the trilepton analysis for the pMSSM models with and without the intermediate sleptons, respectively. The pMSSM models with intermediate sleptons with $M_{1}=100 \mathrm{GeV}$ and $M_{1}=140 \mathrm{GeV}$ showed no improvement from the trilepton analysis, therefore the combination was performed only for the $M_{1}=250 \mathrm{GeV}$ configuration. In both cases, the improvement of the contour of expected and observed upper limit from the individual analyses can be seen. 


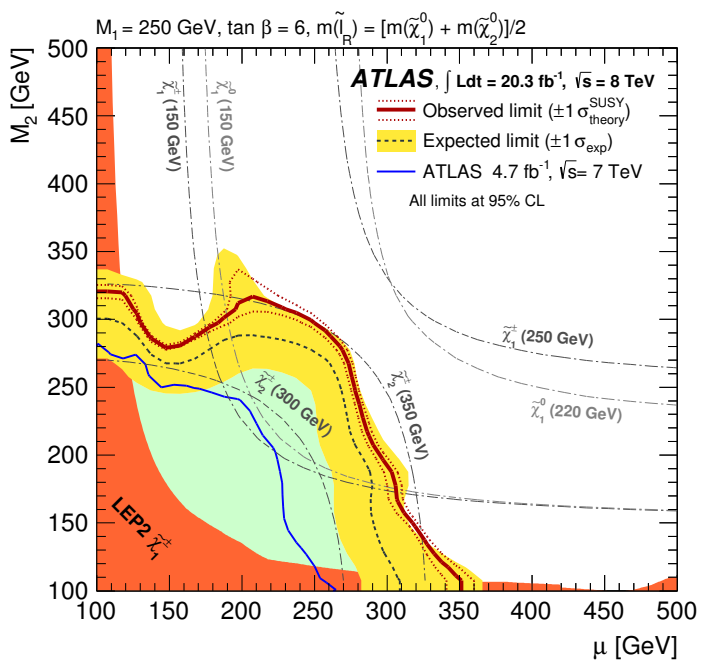

(a)

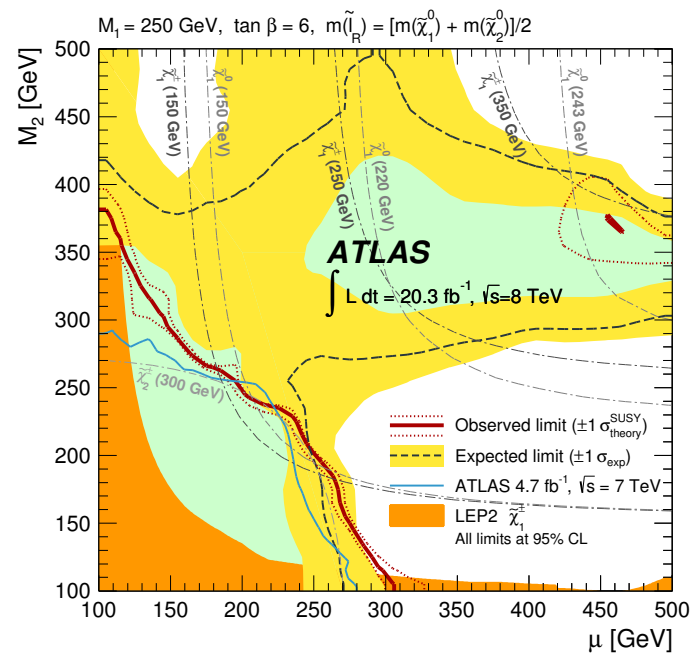

(b)

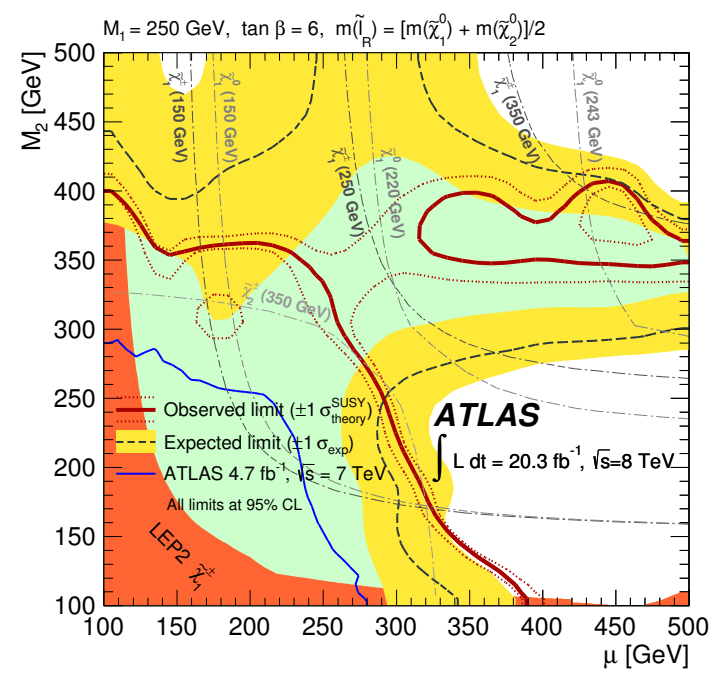

(c)

Figure 6.24: Combined exclusion plot with the trilepton analysis for the pMSSM $D G$ models with intermediate sleptons with the gaugino mass parameter $M_{1}=250 \mathrm{GeV}$. The $\mu$ and $M_{2}$ gaugino mass parameters are varied along the $x$ and the $y$ axis, respectively. The dilepton result (a) and the trilepton result (b) are combined to improve the 95\% CL upper limit (c). The black dotted line with the yellow band represents the expected limit and the thick red line with the dotted red lines represents the observed limit. The pale green area represents the expected excluded region. The blue contour represents the result with the $\sqrt{s}=7$ TeV dataset. 


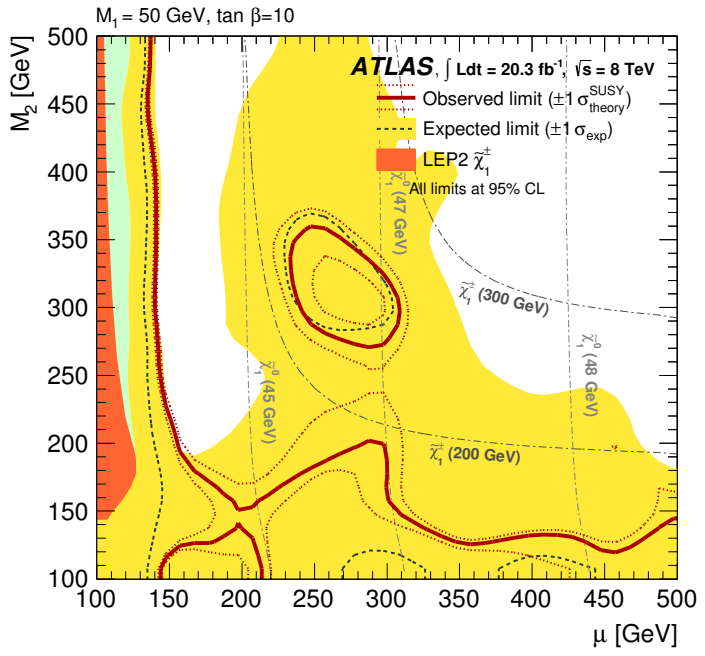

(a)

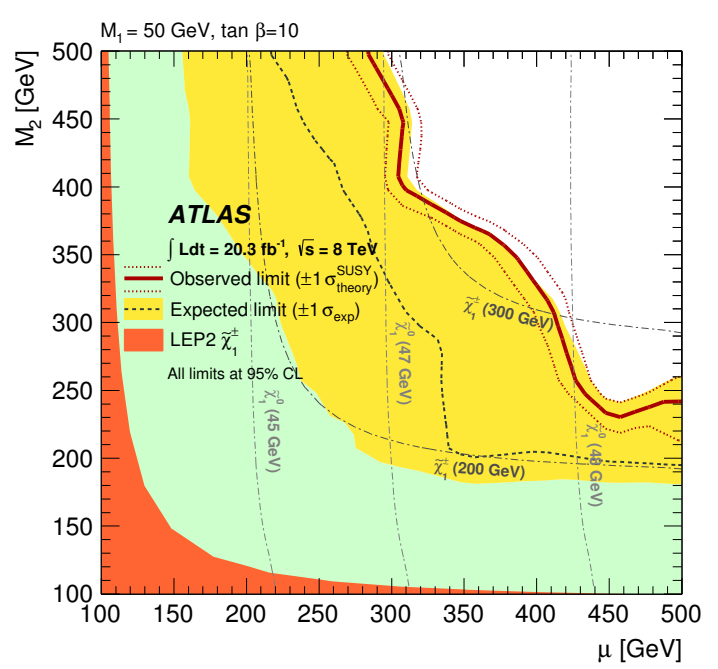

(b)

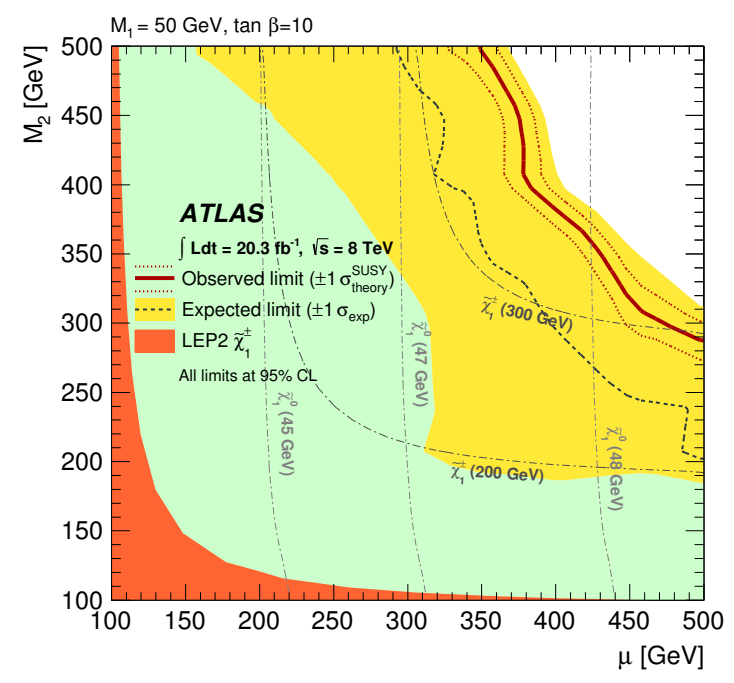

(c)

Figure 6.25: Combined exclusion plot with the trilepton analysis for the pMSSM $D G$ models without intermediate sleptons. The $\mu$ and $M_{2}$ gaugino mass parameters are varied along the $x$ and the $y$ axis, respectively, while $M_{1}$ is fixed at $50 \mathrm{GeV}$. The dilepton result (a) and the trilepton result (b) are combined to improve the $95 \% \mathrm{CL}$ upper limit (c). The black dotted line with the yellow band represents the expected limit and the thick red line with the dotted red lines represents the observed limit. The pale green area represents the expected excluded region. 


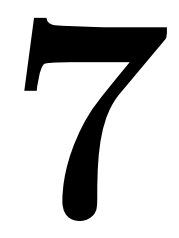

\section{Summary AND Conclusions}

SUSY provides a simple and elegant solution to some of the troubling issues with the SM. Through this thesis work, possible signatures for SUSY processes were explored. In particular, EW SUSY production from $p p$ collisions at the LHC was considered since it is expected to be the dominant mechanism for SUSY production. The ATLAS detector was used to look for possible signatures left by the decaying SUSY particles. For this thesis, the full 2012 data-taking period at the centre-of-mass energy of $8 \mathrm{TeV}$ was used, corresponding to $20.3 \mathrm{fb}^{-1}$ of $p p$ collision data.

This thesis focused on aspects of the published analysis [28] in which I made contributions. No evidence for SUSY signatures was observed as a result of this analysis, but it established the strongest exclusion limits to date on the gaugino mass parameters for the pMSSM models, extending the excluded regions beyond the previously published $7 \mathrm{TeV}$ result as shown in Figures 6.19, 6.20, and 6.21 [86] (except for the pMSSM model without the intermediate sleptons, for which the first exclusion limit was set). The combination of dilepton and trilepton results also saw improvements from the individual analysis. 
The LHC is currently undergoing an upgrade phase, and data-taking will resume in 2015. The upgrades will provide an increased amount of data produced at a significantly higher centre-of-mass collision energy of $13 \mathrm{TeV}$, essential to cover more of the exclusion region, or a possible observation of signatures should SUSY be the true extension to the SM. In the meanwhile, several additional analyses with the 2012 data are still underway in order to extend the SUSY reach. These include: combinations of results from a wider range of analyses (including searches for strongly-produced SUSY); searches with alternate decay channels, such as decays including an intermediate Higgs' boson; and model-specific analyses (for example, searches optimized for pMSSM models). I continue to be involved in these analyses, which should emerge as new publications in peer-reviewed journals, by producing and providing the exclusion results of various models for the EW dilepton case. This is made possible through the expertise that I gained from the endeavour of this thesis work.

The results from this thesis contribute to the overall effort in understanding SUSY, providing exclusions of large regions of the possible phase space for SUSY production. However, even the simplest MSSM model has a large number of parameters over a large range of values that are still unknown. By extending the exclusion regions of these parameters, we may force existing SUSY models to be revisited or even excluded, thereby providing guidance for new theoretical ideas to come forward. 


\section{Personal Contributions to the ATLAS COLlaboration}

Throughout my Ph.D. career, I had the privilege to be a member of the ATLAS collaboration, and I was involved in several service tasks and projects. In this section, my personal contributions and relevant achievements are summarized.

1. LAr Data Investigation and Sign-off (LADIeS)

- Period: Summer 2011

- Relevant chapter: N/A

- Summary of contribution: During the data-taking period in the Summer of 2011, I was involved as a part of the data quality team overlooking the liquid argon (LAr) calorimeter system. The LAr calorimeter system may have temporary problems due to high voltage trips or software errors, and these events need to be flagged in the data-stream as potentially problematic for physics analyses. Daily inspection of collision events were conducted to ensure the quality of the data in terms of the LAr system.

2. Validation of ATLAS Fast-Simulation (AF-II)

- Period: Summer/Fall 2011

- Relevant chapter: Section 4.1.3 
- Summary of contribution: MC simulated samples play crucial roles in all of the physics analyses at ATLAS, but full simulation of the ATLAS detector is computationally heavy, and the collaboration is unable to keep up with the demands for samples (as mentioned in Section 6.5, for the pMSSM model for this thesis work alone, 500 independent samples were needed). AF-II replaces the full simulation of the calorimeter system by parameterizing the energy deposited in the calorimeter by the collisions. This parameterization needs to be validated. I contributed to this validation process by looking at the low-energy regions with a large population of the $J / \Psi$ mesons which are one of the benchmark processes for lepton production.

3. D3PD Production for e/gamma Performance Group

- Period: Summer 2012 - Spring 2014

- Relevant chapter: Section 2.2.1

- Summary of contribution: As described in Section 2.2.1, the D3PD is a user-analysis data format for both collision and MC simulated data, readable by the CERN ROOT framework. They are centrally produced by the physics and performance groups within the ATLAS collaboration, and I took on the responsibilities to produce them for the e/gamma performance group. The tasks included requesting the production of the MC samples for various reasons (new model, fix for the known bugs or modified parameters, or samples requiring more events to reduce the statistical uncertainties). For the collision data, I was also responsible for making a data "container", which is a collection of ATLAS data runs which are categorized into a period with certain data-taking conditions.

4. Development of SUSY Analysis Package

- Period: 2013 - 2014

- Relevant chapter: Section 4.2

- Summary of contribution: SUSY analyses within the ATLAS collaboration use a common analyses package called SUSYTools. This package 
unifies the definitions of physics objects used for analyses, and it is under constant evolution due to various inputs and recommendations from relevant performance groups. I was involved in adding features that are relevant to the wider range of SUSY analyses, and updating the code as necessary (bug fixes).

5. Electroweak SUSY Searches with Two-Lepton Final States

- Period: 2011 - 2014

- Relevant chapter or reference: Chapter 6

- Summary of contribution: Since the beginning of my Ph.D. degree, I was involved in the SUSY searches with two-lepton final states. The details of the analysis has evolved throughout the years, and correspondingly the results have also improved, as it can be seen in the results presented in Chapter 6. I have made personal contributions to the analyses presented in the following references $[28,86,76]$. 


\section{List of Presentations Given}

Throughout my Ph.D. career, I have given several presentations on my research to various audiences outside of the SUSY working group. A compiled list of these presentations is given in this section.

- Title: Improving the Dilepton + Dijet SUSY Signal Region for Direct Gaugino Production

- Location (Date): ATLAS Canada Workshop - TRIUMF, Vancouver (December 13, 2011)

- Summary: Presented my contributions to the SUSY search analysis for $\sqrt{s}=7 \mathrm{TeV}$ data

- Title: Searches for Electroweak SUSY Signatures with Two Leptons and Missing Energy with the ATLAS Detector

- Location (Date): Ottawa-Carleton Institute for Physics (OCIP) Presentations - Ottawa (December 10, 2013)

- Summary: Presented limited results of the analysis, removing sensitive results that were not approved by the ATLAS collaboration at the time

- Title: Charginos, neutralinos, and sleptons with 2 leptons and $E_{\mathrm{T}}^{\text {miss }}$ 
- Location (Date): Public Approval meeting - CERN (January 28, 2014)

- Summary: Presented summary at the public reading (within the ATLAS collaboration) of the EW SUSY analysis to be published. This presentation is an internal ATLAS requirement before the submission of an analysis to a journal for publication.

- Title: Searches for Electroweak SUSY Signatures with Two Leptons and Missing Energy with the ATLAS Detector

- Location (Date): Winter Nuclear and Particle Physics Conference (WNPPC) - Banff (February 14, 2014)

- Summary: Presented limited results of the analysis, removing sensitive results that were not approved by the ATLAS collaboration at the time

- Title: Search for charginos, neutralinos and sleptons in 1 or 2 leptons final state events with ATLAS detector (Poster)

- Location (Date): LHC Experiments Committee - CERN (March 5, 2014)

- Summary: Presented results of the EW SUSY analysis alongside similar SUSY searches with 1 lepton and $b b$ signature. 


\section{List OF ACRONYMS}

\begin{tabular}{ll} 
ALICE & A Large Ion Collider Experiment \\
ATLAS & A Toroidal LHC Apparatus \\
BDT & Boosted Decision Tree \\
BSM & Beyond the Standard Model \\
CDF & Cumulative Distribution Function \\
CERN & Conseil Européenne pour la Recherche Nucléaire \\
CL & Confidence Level \\
CMS & Compact Muon Solenoid \\
CP & Charge-conjugation/Parity \\
CR & Control Region \\
CSC & Cathode Strip Chamber \\
DF & Different Flavour \\
DG & Direct Gaugino \\
EDM & Estimated Distance to Minimum \\
EER & Electron Energy Resolution \\
\hline
\end{tabular}




\begin{tabular}{|c|c|}
\hline EES & Electron Energy Scale \\
\hline EF & Event Filter \\
\hline EM & Electromagnetic \\
\hline EW & Electroweak \\
\hline GRL & Good Runs List \\
\hline ID & Inner Detector \\
\hline JER & Jet Energy Resolution \\
\hline JES & Jet Energy Scale \\
\hline JVF & Jet Vertex Fraction \\
\hline $\mathbf{L 1}$ & Level-1 (trigger) \\
\hline $\mathbf{L} 2$ & Level-2 (trigger) \\
\hline LAr & Liquid Argon \\
\hline LC & Local Cluster \\
\hline LEP & Large Electron-Positron (Collider) \\
\hline LHC & Large Hadron Collider \\
\hline LHCb & LHC-beauty \\
\hline LSP & Lightest Supersymmetric Particle \\
\hline MC & Monte Carlo \\
\hline MDT & Monitored Drift Tube \\
\hline MS & Muon Spectrometer \\
\hline MSSM & Minimally Supersymmetric Standard Model \\
\hline mSUGRA & Minimal Supergravity \\
\hline PDF & Parton Distribution Function \\
\hline pMSSM & phenomenological MSSM \\
\hline
\end{tabular}




\begin{tabular}{|c|c|}
\hline PS & Proton Synchrotron \\
\hline PSB & PS Booster \\
\hline PV & Primary Vertex \\
\hline QCD & Quantum Chromodynamics \\
\hline QED & Quantum Electrodynamics \\
\hline ROI & Region of Interest \\
\hline RPC & Resistive Plate Chamber \\
\hline $\mathrm{SCT}$ & Semiconductor Tracker \\
\hline SM & Standard Model \\
\hline SPS & Super Protron Synchrotron \\
\hline SF & Scale Factor / Same Flavour \\
\hline SR & Signal Region \\
\hline SUSY & Supersymmetry \\
\hline SV & Secondary Vertex \\
\hline TGC & Thin Gap Chamber \\
\hline TRT & Transition Radiation Tracker \\
\hline TTC & Trigger-timing Control \\
\hline UE & Underlying Event \\
\hline VBF & Vector Boson Fusion \\
\hline VEV & Vacuum Expectation Value \\
\hline
\end{tabular}




\section{REFERENCES}

[1] ATLAS Collaboration, Observation of a new particle in the search for the Standard Model Higgs boson with the ATLAS detector at the LHC, Phys.Lett. B716 (2012) 1-29, [arXiv: 1207.7214].

[2] CMS Collaboration, Observation of a new boson at a mass of $125 \mathrm{GeV}$ with the CMS experiment at the LHC, Phys.Lett. B716 (2012) 30-61, [arXiv: 1207.7235].

[3] Particle Data Group Collaboration, J. Beringer et al., Review of Particle Physics, Phys.Rev. D86 (2012) 010001.

[4] S. Tomonaga, On a relativistically invariant formulation of the quantum theory of wave fields, Prog.Theor.Phys. 1 (1946) 27-42.

[5] R. P. Feynman, Space-time approach to quantum electrodynamics, Phys.Rev. 76 (1949) 769-789.

[6] J. Schwinger, Quantum electrodynamics. $i$. a covariant formulation, Phys.Rev. 74 (1948) 1439-1461.

[7] M. Y. Han and Y. Nambu, Three-triplet model with double SU(3) symmetry, Phys.Rev. 139 (1965) B1006-B1010.

[8] J. D. Bjorken, Asymptotic sum rules at infinite momentum, Phys.Rev. 179 (1969) 1547-1553. 
[9] D. J. Gross and F. Wilczek, Asymptotically free gauge theories. i, Phys.Rev. D8 (1973) 3633-3652.

[10] S. L. Glashow, Partial Symmetries of Weak Interactions, Nucl.Phys. 22 (1961) $579-588$.

[11] S. Weinberg, Conceptual Foundations of the Unified Theory of Weak and Electromagnetic Interactions, Rev.Mod.Phys. 52 (1980) 515-523.

[12] A. Salam, Gauge Unification of Fundamental Forces, Rev.Mod.Phys. 52 (1980) $525-538$.

[13] P. W. Higgs, Broken symmetries, massless particles and gauge fields, Phys.Lett. 12 (1964) 132-133.

[14] S. P. Martin, A Supersymmetry Primer, hep-ph/9709356.

[15] N. Arkani-Hamed, S. Dimopoulos, and G. R. Dvali, The Hierarchy problem and new dimensions at a millimeter, Phys.Lett. B429 (1998) 263-272, [hep-ph/9803315].

[16] ATLAS Collaboration, Measurement of the Higgs boson mass from the $H \rightarrow \gamma \gamma$ and $H \rightarrow Z Z^{*} \rightarrow 4 \ell$ channels with the ATLAS detector using $25 \mathrm{fb}^{-1}$ of pp collision data, arXiv:1406.3827.

[17] M. E. Peskin and D. V. Schroeder, An Introduction to Quantum Field Theory. Advanced book classics. Addison-Wesley Publishing Company, 1995.

[18] K. G. Begeman, A. H. Broeils, and R. H. Sanders, Extended rotation curves of spiral galaxies: dark haloes and modified dynamics, Monthly Notices of the Royal Astronomical Society 249 (1991), no. 3 523-537.

[19] H. E. Haber and G. L. Kane, The search for supersymmetry: Probing physics beyond the standard model, Phys.Rep. 117 (1985), no. 2-4 75 - 263.

[20] I. J. R. Aitchison, Supersymmetry and the MSSM: An Elementary Introduction, hep-ph/0505105. 
[21] LEP2 SUSY Working Group, "Combined LEP chargino results, up to $208 \mathrm{GeV}$, for large $m_{0}$." [http://lepsusy . web.cern.ch/lepsusy/www/inos_moriond01/ charginos_pub.html (Accessed: 2014-12-29)].

[22] CDF and D0 Collaboration, SUSY searches at the Tevatron, EPJ Web Conf. 28 (2012) 09006, [arXiv:1202.0712].

[23] R. Bailey, C. Benvenuti, S. Myers, and D. Treille, The LEP collider, Comptes Rendus Physique 3 (2002), no. 91107 - 1120.

[24] Fermilab, "Tevatron accelerator." [http://www.fnal.gov/pub/tevatron/tevatron-accelerator.html (Accessed: 2014-12-29)].

[25] R. W. Assmann, M. Lamont, and S. Myers, A Brief History of the LEP Collider, Nucl.Phys.B, Proc.Suppl. 109 (2002), no. CERN-SL-2002-009-OP 17-31. $15 \mathrm{p}$.

[26] L. Evans, (ed. ) and P. Bryant, (ed. ), LHC Machine, JINST 3 (2008) S08001.

[27] ATLAS Collaboration, The ATLAS Experiment at the CERN Large Hadron Collider, JINST 3 (2008) S08003.

[28] ATLAS Collaboration, Search for direct production of charginos, neutralinos and sleptons in final states with two leptons and missing transverse momentum in pp collisions at $\sqrt{s}=8 \mathrm{TeV}$ with the ATLAS detector, JHEP 05 (2014) 071, [arXiv: 1403.5294].

[29] ALICE Collaboration, The ALICE experiment at the CERN LHC, JINST 3 (2008) S08002.

[30] CMS Collaboration, The CMS experiment at the CERN LHC, JINST 3 (2008) S08004.

[31] LHCb Collaboration, The LHCb Detector at the LHC, JINST 3 (2008) S08005. 
[32] J. M. Butterworth, G. Dissertori, and G. P. Salam, Hard Processes in Proton-Proton Collisions at the Large Hadron Collider, Ann.Rev.Nucl.Part.Sci. 62 (2012) 387-405, [arXiv: 1202.0583].

[33] ATLAS Collaboration, ATLAS detector and physics performance: Technical Design Report, 1. Technical Design Report ATLAS. CERN, Geneva, 1999. CERN-LHCC-99-014 [http://cds. cern.ch/record/391176].

[34] ATLAS Event Display Twiki. [https:

//twiki.cern.ch/twiki/bin/view/AtlasPublic/EventDisplayStandAlone (Accessed: 2014-12-29)].

[35] R. Brun and F. Rademakers, ROOT An Object Oriented Data Analysis Framework, Nucl.Inst.Meth. A389 (1997), no. 1-2 81 - 86.

[36] Data Acquisition and Luminosity Plots Twiki. [https:

//twiki.cern.ch/twiki/bin/view/AtlasPublic/LuminosityPublicResults (Accessed: 2014-12-29)].

[37] W. R. Hamilton, On a general method in dynamics, Phil.Trans.R.Soc.Lond. 124 (1834) 247-308.

[38] W. R. Hamilton, Second essay on a general method in dynamics, Phil.Trans.R.Soc.Lond. 125 (1835) 95-144.

[39] D. J. H. Chung et al., The Soft supersymmetry breaking Lagrangian: Theory and applications, Phys.Rept. 407 (2005) 1-203, [hep-ph/0312378].

[40] A. Djouadi, The anatomy of electroweak symmetry breaking Tome II: The Higgs bosons in the Minimal Supersymmetric Model, Phys.Rep. 459 (2008), no. 1-6 1 $-241$.

[41] A. Djouadi, The anatomy of electroweak symmetry breaking: Tome I: The Higgs boson in the Standard Model, Phys.Rep. 457 (2008), no. 1-4 1- 216.

[42] LHC SUSY Cross Section Working Group Twiki. [https://twiki.cern.ch/twiki/bin/view/LHCPhysics/SUSYCrossSections (Accessed: 2014-12-29)]. 
[43] Summary plots from the ATLAS Supersymmetry physics group Twiki. [https://atlas.web.cern.ch/Atlas/GROUPS/PHYSICS/ CombinedSummaryPlots/SUSY/index.html (Accessed: 2014-12-29)].

[44] M. Papucci, J. T. Ruderman, and A. Weiler, Natural SUSY Endures, JHEP 1209 (2012) 035, [arXiv:1110.6926].

[45] ATLAS Collaboration, Search for electroweak production of supersymmetric particles in final states with at least two hadronically decaying taus and missing transverse momentum with the ATLAS detector in proton-proton collisions at $\sqrt{s}=8 \mathrm{TeV}$, ATLAS-CONF-2013-028 [http://cds.cern.ch/record/1525889].

[46] C. F. Berger et al., Supersymmetry Without Prejudice, JHEP 0902 (2009) 023, [arXiv:0812.0980].

[47] GEANT4 Collaboration, S. Agostinelli et al., GEANT4: A simulation toolkit, Nucl.Instrum.Meth. A506 (2003) 250-303.

[48] ATLAS Collaboration, The ATLAS Simulation Infrastructure, Eur.Phys.J. C70 (2010) 823-874, [arXiv: 1005.4568].

[49] S. Frixione and B. R. Webber, Matching NLO QCD computations and parton shower simulations, JHEP 0206 (2002) 029, [hep-ph/0204244].

[50] J. M. Butterworth, J. R. Forshaw, and M. H. Seymour, Multiparton interactions in photoproduction at HERA, Z.Phys. C72 (1996) 637-646, [hep-ph/9601371].

[51] S. Frixione, P. Nason, and C. Oleari, Matching NLO QCD computations with Parton Shower simulations: the POWHEG method, JHEP 0711 (2007) 070, [arXiv: 0709.2092].

[52] T. Sjostrand, S. Mrenna, and P. Skands, A Brief Introduction to PYTHIA 8.1, Comput.Phys.Commun. 178 (2008) 852-867, [arXiv:0710.3820].

[53] T. Binoth, M. Ciccolini, N. Kauer, and M. Kramer, Gluon-induced W-boson pair production at the LHC, JHEP 0612 (2006) 046, [hep-ph/0611170]. 
[54] T. Gleisberg et al., Event generation with SHERPA 1.1, JHEP 0902 (2009) 007, [arXiv:0811.4622].

[55] T. Binoth, N. Kauer, and P. Mertsch, Gluon-induced QCD corrections to $p p \rightarrow$ $Z Z \rightarrow \ell \bar{\ell} \ell^{\prime} \bar{\ell}^{\prime}$, arXiv:0807.0024.

[56] J. Alwall et al., MadGraph 5 : Going Beyond, JHEP 1106 (2011) 128, [arXiv: 1106.0522].

[57] T. Sjostrand, S. Mrenna, and P. Skands, PYTHIA 6.4 physics and manual, JHEP 5 (2006) 026.

[58] M. L. Mangano et al., ALPGEN, a generator for hard multiparton processes in hadronic collisions, JHEP 0307 (2003) 001, [hep-ph/0206293].

[59] M. Bahr et al., Herwig ++ Physics and Manual, Eur.Phys.J. C58 (2008) 639-707, [arXiv:0803.0883].

[60] S. Gieseke, C. Rohr, and A. Siodmok, Colour reconnections in Herwig ++ , Eur.Phys.J. C72 (2012) 2225, [arXiv:1206.0041].

[61] ATLAS Collaboration, Search for direct production of charginos and neutralinos in events with three leptons and missing transverse momentum in $\sqrt{s}=8 T e V$ pp collisions with the ATLAS detector, JHEP 1404 (2014) 169, [arXiv: 1402 .7029].

[62] ATLAS Collaboration, Expected Performance of the ATLAS Experiment Detector, Trigger and Physics, arXiv:0901.0512. CERN-OPEN-2008-020.

[63] ATLAS Collaboration, Electron efficiency measurements with the ATLAS detector using the 2012 LHC proton-proton collision data, ATLAS-CONF-2014-032 [http://cds.cern.ch/record/1706245].

[64] ATLAS Collaboration, Muon Momentum Resolution in First Pass Reconstruction of pp Collision Data Recorded by ATLAS in 2010, ATLAS-CONF-2011-046 [http://cds . cern.ch/record/1338575]. 
[65] ATLAS Collaboration, Measurement of the muon reconstruction performance of the ATLAS detector using 2011 and 2012 LHC proton-proton collision data, Eur.Phys.J. C74 (2014), no. 11 3130, [arXiv:1407.3935].

[66] ATLAS Collaboration, Identification and energy calibration of hadronically decaying tau leptons with the ATLAS experiment in pp collisions at $\sqrt{s}=8 \mathrm{TeV}$, arXiv: 1412.7086.

[67] M. Cacciari, G. P. Salam, and G. Soyez, The Anti-k(t) jet clustering algorithm, JHEP 0804 (2008) 063, [arXiv:0802.1189].

[68] ATLAS Collaboration, Jet energy measurement with the ATLAS detector in proton-proton collisions at $\sqrt{s}=7 \mathrm{TeV}$, Eur.Phys.J. C73 (2013) 2304, [arXiv: 1112.6426].

[69] ATLAS Collaboration, Measuring the b-tag efficiency in a top-pair sample with $4.7 \mathrm{fb}^{-1}$ of data from the ATLAS detector, ATLAS-CONF-2012-097 [http://cds.cern.ch/record/1460443].

[70] ATLAS Collaboration, Measurement of the Mistag Rate with $5 \mathrm{fb}^{-1}$ of Data Collected by the ATLAS Detector, ATLAS-CONF-2012-040 [http://cds.cern.ch/record/1435194].

[71] ATLAS Collaboration, Performance of Missing Transverse Momentum Reconstruction in Proton-Proton Collisions at $7 \mathrm{TeV}$ with ATLAS, Eur.Phys.J. C72 (2012) 1844, [arXiv:1108.5602].

[72] ATLAS Collaboration, Jet energy resolution in proton-proton collisions at $\sqrt{s}=7 \mathrm{TeV}$ recorded in 2010 with the ATLAS detector, Eur.Phys.J. C73 (2013) 2306, [arXiv:1210.6210].

[73] ATLAS Collaboration Collaboration, Electron and photon energy calibration with the ATLAS detector using LHC Run 1 data, Eur.Phys.J. C74 (2014), no. 10 3071, [arXiv:1407.5063]. 
[74] ATLAS Collaboration, Electron reconstruction and identification efficiency measurements with the ATLAS detector using the 2011 LHC proton-proton collision data, Eur.Phys.J. C74 (2014) 2941, [arXiv:1404.2240].

[75] ATLAS Collaboration, Preliminary results on the muon reconstruction efficiency, momentum resolution, and momentum scale in ATLAS $2012 \mathrm{pp}$ collision data, ATLAS-CONF-2013-088 [http://cds.cern.ch/record/1580207].

[76] ATLAS Collaboration, Searches for direct slepton-pair and chargino-pair production in final states with two opposite-sign leptons, missing transverse momentum and no jets in $20 \mathrm{fb}^{-1}$ of pp collisions at $\sqrt{s}=8 \mathrm{TeV}$ with the ATLAS detector, ATLAS-CONF-2013-049 [https://cds.cern.ch/record/1547565].

[77] ATLAS Collaboration, Improved luminosity determination in pp collisions at $\sqrt{s}=7 \mathrm{TeV}$ using the ATLAS detector at the LHC, Eur.Phys.J. C73 (2013) 2518, [arXiv: 1302.4393].

[78] ATLAS Collaboration, Measurements of the properties of the Higgs-like boson in the $W W^{(*)} \rightarrow \ell \nu \ell \nu$ decay channel with the ATLAS detector using $25 \mathrm{fb}^{-1}$ of proton-proton collision data, ATLAS-CONF-2013-030 [https://cds.cern.ch/record/1527126].

[79] C. G. Lester and D. J. Summers, Measuring masses of semi-invisibly decaying particle pairs produced at hadron colliders, Phys.Lett. B463 (1999), no. 199 103.

[80] G. Arnison et al., Experimental observation of isolated large transverse energy electrons with associated missing energy at $\sqrt{s}=540 \mathrm{GeV}$, Phys.Lett. B122 (1983), no. $1103-116$.

[81] G. Cowan et al., Asymptotic formulae for likelihood-based tests of new physics, Eur.Phys.J. C71 (2011), no. 2 1-19. 
[82] R. D. Cousins, J. T. Linnemann, and J. Tucker, Evaluation of three methods for calculating statistical significance when incorporating a systematic uncertainty into a test of the background-only hypothesis for a Poisson process, Nucl.Inst.Meth. A595 (2008), no. 2480 - 501.

[83] G. Cowan, Statistical Data Analysis. Oxford University Press, Oxford, 1998.

[84] M. Baak et al., HistFitter software framework for statistical data analysis, arXiv:1410.1280.

[85] ATLAS VP1. [https://atlas-vp1.web.cern.ch/atlas-vp1/home/ (Accessed: 2014-12-29)].

[86] ATLAS Collaboration, Search for direct slepton and gaugino production in final states with two leptons and missing transverse momentum with the ATLAS detector in pp collisions at $\sqrt{s}=7$ TeV, Phys.Lett. B718 (2013) 879-901, [arXiv: 1208. 2884]. 